\title{
Development of Data Sheets for Statistical Evaluation of
}

\section{Fatigue Data}

The topic of this Master's Thesis was approved by the Head of the Degree Programme of the Department of Mechanical Engineering on March 12, 2007

Supervisors: Prof. Gary Marquis and Dr. Timo Björk Examiners: Prof. Gary Marquis and Dr. Timo Björk

$12^{\text {th }}$ of March 2007

Jia Yu

RUSKONLAHDENK. 13-15 C8

53850 LAPPEENRANTA

Phone +358 509240454 
ABSTRACT

Jia Yu

Development of Data Sheets for Statistical Evaluation of Fatigue Data

Lappeenranta University of Technology

Mechanical Engineering Department

Examiners: Prof. Gary Marquis and Dr. Timo Björk

Master's thesis 2007

103 pages, 12 figures, 2 tables and 12 appendixes

To enable a mathematically and physically sound execution of the fatigue test and a correct interpretation of its results, statistical evaluation methods are used to assist in the analysis of fatigue testing data. The main objective of this work is to develop step-by-step instructions for statistical analysis of the laboratory fatigue data. The scope of this project is to provide practical cases about answering the several questions raised in the treatment of test data with application of the methods and formulae in the document IIW-XIII-2138-06 (Best Practice Guide on the Statistical Analysis of Fatigue Data). Generally, the questions in the data sheets involve some aspects: estimation of necessary sample size, verification of the statistical equivalence of the collated sets of data, and determination of characteristic curves in different cases. The series of comprehensive examples which are given in this thesis serve as a demonstration of the various statistical methods to develop a sound procedure to create reliable calculation rules for the fatigue analysis.

Keywords: fatigue, verification, statistical equivalence, data sheets 


\section{ACKNOWLEDGEMENT}

The thesis work was carried out at the laboratory of Fatigue and Strength, in the Department of Mechanical Engineering, Faculty of Technology, Lappeenranta University of Technology during the period from October 2006 to March 2007. The thesis formed a part of development in statistical evaluation of fatigue data.

First of all, I wish to give my sincere thanks to my supervisors, Professor Gary Marquis and Doctor Timo Björk, Lappeenranta University of Technology, for their valuable comments and advice as well as enthusiastic support and patience at all stages of my work.

I also would like to thank all my colleagues, Li Tong, Martin Mbeng, in the same office at Lappeenranta University of Technology for their assistance and suggestions.

Finally I am thankful to my parents for their love and encouragement to support me to finish my thesis project.

Lappeenranta, March, 2007.

Jia Yu 


\section{TABLE OF CONTENTS}

ABSTRACT 2

ACKNOWLEDGEMENT 3

TABLE OF CONTENTS 4

NOMENCLATRURE 6

1 INTRODUCTION 8

2 FATIGUE TESTING 11

2.1 The relationship between stress range and cycles 11

$\begin{array}{ll}2.2 \text { The log-normal distribution } & 11\end{array}$

$\begin{array}{ll}2.3 \text { Statistical independent results } & 13\end{array}$

$\begin{array}{lll}3 & \text { FITTING A S-N CURVE } & 14\end{array}$

$\begin{array}{ll}3.1 \text { Fatigue data } & 15\end{array}$

$\begin{array}{ll}\text { 3.2 Methods of estimating S-N curve parameters } & 17\end{array}$

$\begin{array}{lc}3.21 \text { Least squares regression method } & 18\end{array}$

$\begin{array}{ll}3.22 \text { Maximum likelihood regression method } & 19\end{array}$

$\begin{array}{ll}\text { 3.3 Sample size and population } & 20\end{array}$

4 ESTABLISHING A DESIGN CURVE 22

4.1 Mean, characteristic and design values 22

$\begin{array}{ll}4.2 \text { Confidence limits } & 23\end{array}$

$\begin{array}{ll}4.3 \text { Prediction limits } & 25\end{array}$

4.4 Tolerance limits $\quad 26$

$\begin{array}{ll}4.5 \text { Comparison } & 28\end{array}$

5 VERIFICATION OF THE STATISTICAL EQUIVALENCE FOR TWO 30 S-N CURVES

$\begin{array}{ll}5.1 \text { Tests performed to an S-N curve } & 30\end{array}$

6 VERIFICATION OF WEIBULL DISTRIBUTED DATA 33

$\begin{array}{ll}\text { 6.1 Weibull probability graph } & 33\end{array}$ 
6.2 Determine the shape parameter and scale parameter

6.3 A goodness of fit test: Anderson-Darling 36

7 DETERMINE THE IMPROVEMENT FROM POST-WELD TREATMET 38

$\begin{array}{ll}\text { 7.1 Post-weld treatment } & 38\end{array}$

7.2 Statistical analysis of the test data 38

8 DATA SHEETS: APPLICATION OF STATISTICAL ANALYSIS OF 40 FATIGUE

$9 \quad$ CONCLUSION AND RECOMMENDATIONS 42

$\begin{array}{ll}\text { REFERENCES } & 44\end{array}$

$\begin{array}{ll}\text { APPENDIXES } & 46\end{array}$

$\begin{array}{llr}\text { Appendix I } & 47\end{array}$

$\begin{array}{ll}\text { Appendix II } & 53\end{array}$

$\begin{array}{ll}\text { Appendix III } & 57\end{array}$

$\begin{array}{ll}\text { Appendix IV } & 61\end{array}$

$\begin{array}{ll}\text { Appendix V } & 66\end{array}$

$\begin{array}{ll}\text { Appendix VI } & 72\end{array}$

$\begin{array}{ll}\text { Appendix VII } & 77\end{array}$

$\begin{array}{ll}\text { Appendix VIII } & 84\end{array}$

$\begin{array}{lr}\text { Appendix IX } & 92\end{array}$

$\begin{array}{ll}\text { Appendix X } & 100\end{array}$

$\begin{array}{ll}\text { Appendix XI } & 102\end{array}$

$\begin{array}{ll}\text { Appendix XII } & 103\end{array}$ 
$\alpha$

A

$\beta$

$\eta$

$\gamma$

C

$C_{\mu}$

$C_{\sigma}$

$\mathrm{Cr}$

E

F

$h, \chi^{2}$

$k$

$k_{c}$

$m$

$\lambda$

$\mu$

$n$

$N$

$N_{p}$

$P$
Significance level

Intercept of the regression line

Shape parameter of Weibull distribution

Scale parameter of Weibull distribution

Location parameter of Weibull distribution, significance

level of confidence limits

Fatigue capacity

Confidence level applied on the population mean

Confidence level applied on the population variance

Criteria of hypothesis test

Parameter of Multiple non-linear regression,

Percentage point of the $F$ distribution, Parameter of Multiple non-linear regression

Independent variable in the chi-square distribution

One sided tolerance limit factor

Confidence limit factor

Slope exponent of the S-N curve

Independent variable in the standardized normal distribution

Population mean of a continuous random variable

Number of specimens

Life in number of cycles from the S-N curve

Life related to failure probability $P$

Probability of failure 


\begin{tabular}{|c|c|}
\hline$R$ & Reliability, stress ratio, variance ratio \\
\hline$S$ & Sample standard deviation \\
\hline$S$ & Stress range \\
\hline$S_{a}$ & Stress amplitude \\
\hline$S_{m}$ & Mean stress \\
\hline$S_{\max }$ & $\begin{array}{l}\text { Maximum stress equal to the sum of the mean and the } \\
\text { amplitude of an alternating stress }\end{array}$ \\
\hline${ }^{\mathrm{s}} \mathrm{S}$ & Standard deviation \\
\hline$\Delta S$ & Stress range \\
\hline$\sigma$ & Stress, population standard deviation \\
\hline$\hat{\sigma}^{2}$ & $\begin{array}{l}\text { The best estimate of the variance of the data about } \\
\text { regression line }\end{array}$ \\
\hline$\sigma_{a}$ & Stress amplitude \\
\hline$\sigma_{e}$ & Estimate of the common variance of two samples \\
\hline$t$ & Independent variable in the Student's t distribution \\
\hline$T$ & Difference between two estimated slops \\
\hline$x$ & Continuous random variable \\
\hline $\bar{x}$ & Sample mean \\
\hline$x_{k}$ & Characteristic value \\
\hline$X$ & Difference between two estimated intercept \\
\hline$\Phi(x)$ & Distribution function. Probability density integral \\
\hline$\delta$ & Specified precision of the estimate \\
\hline$Z_{\alpha}$ & $\begin{array}{l}\text { Ordinate on the normal curve corresponding to significant } \\
\text { level }\end{array}$ \\
\hline
\end{tabular}




\section{INTRODUCTION}

Components of machines are frequently subjected to cyclic loads, and the resulting cyclic stresses can lead to microscopic physical damage. The process of damage and failure due to cyclic loading is called fatigue. In practice, Mechanical structures often have defects or stress concentrations which cause cracks to nucleate and propagate. Even very small stress concentration areas can lead to fatigue failure of dynamically loaded structures. Therefore, the fatigue failures have been highly concerned in engineering design.

Fatigue tests are made with the objective of determining the relationship between the stress range and the number of stress cycles applied before causing failure. It is implemented to determine basic material or material joining characteristics such as: fatigue limit, S-N fatigue strength curves and crack propagation curves. It based on the relationship between the fatigue resistance of a given material, component or structural detail and cyclic load. The testing samples are usually subjected to cyclically varying stresses which may be tension, compression, torsion and bending or a combination of these stresses.

In fatigue tests, different test specimens and testing conditions make the observed results distinct and invariably scattered. Consequently, statistical methods are used to establish the required relationship between applied load and the number of cycles to failure. Statistical methods are available to assist in the analysis of fatigue testing data. For the non-expert, these are often difficult to apply.

With the increasing use of fatigue testing to supplement design rules, the IIW commission of fatigue of welded components and structures has developed a Best Practice Guide on Statistical Analysis of Fatigue Data (Schneider and Maddox, 2006). It focuses on fatigue endurance test results obtained under constant amplitude load. The analyses are concerned purely with the experimental data. They are independent of the tested material. 
This current work is based on this Best Practice Guide. The goal has been to develop data sheets to solve a series of practical questions in the analysis of fatigue. The objective of this work is to provide step-by-step recommendations on the use for analyzing fatigue data. The scope of this guidance is to provide practical cases to answer several important questions raised regarding the treatment of test result. Generally, the questions involved the estimation of necessary sample size, verification of the statistical equivalence of the collated sets of data, and determination of characteristic curves in different cases.

Working group 1 of Commission XIII in the International Institute of Welding is currently developing a series of working sheets in order to help non-experts apply the statistical principals outlined in the best practice guidance document [IIW.XIII-WG1-121r3-06] (International institute of welding, 2006). The goal is that works sheets on the following questions should be developed:

1. Can two data sets be merged?

2. Are the variances of 2 data sets statistically equivalent?

3. Are the means of 2 data sets statistically equivalent?

4. Are the data normal distributed using a normal probability graph (Henry graph)

5. Are the data normal distributed using a likelihood test?

6. Are the data Weibull distributed using a Weibull probability graph?

7. Does a standard S-N curve fit with a data set?

8. How many results are necessary to validate a selected S-N curve?

9. What S-N curve can be selected from a set of non cracked samples results?

10. How to determine a design S-N curve, slope fixed (prediction limits)?

11. How to determine a design S-N curve, slope estimated (prediction limits)?

12. How to determine a design S-N curve, slope fixed (tolerance limits)?

13. How to determine a design S-N curve, slope estimated (tolerance limits)?

14. How to determine a mean S-N curve, Bastenaire equation?

15. Are 2 experimental design S-N curves statistically equivalent? 
Additionally, Lappeenranta University of Technology is interested in post weld improvement technologies; therefore, one additional question has been added.

16. How to determine the degree of improvement produced by a post-weld treatment process?

Draft versions of worksheets for questions 1 through 5 and question 7 have been developed previously. The specific goal of this thesis is to develop draft worksheets for questions 6, 8 through 13, 15 and 16. 


\section{FATIGUE TESTING}

\subsection{The relationship between stress range and cycles}

The results of fatigue tests from a number of different stress levels can be plotted to obtain a stress-life curve, also called an S-N curve. The amplitude of local stress or nominal stress, $\sigma_{a}$ or $S_{a}$ is commonly plotted versus the number of cycles to failure $N_{f}$.

In most cases, test results are obtain at constant stress ratios, $R$. The S-N data is presented in a graph showing logarithm of endurance in cycles as the abscissa and logarithm of stress range as the ordinate. There is an underlying linear relationship between $\log S$ and $\log N$ of the form:

$\log N=\log A-m \log S$

where: $m$ is the slope and $\log A$ is the intercept

Another rewritten form is commonly used to describe S-N curves in design rules:

$S^{m} N=A$

The lower limit on $S$ is determined by the fatigue endurance limit. Commonly, it is chosen on the basis of endurance that can be achieved without fatigue cracking, typically between $N=2 \times 10^{6}$ and $10^{7}$ cycles. While, the upper limit on $S$ is taken to be the maximum allowable static design stress.

\subsection{The log-normal distribution}

In statistics, a probability distribution describes how probabilities are distributed on events. The probability distribution is used to help us estimate the population by using limited samples. According to different types of random variables, the probability distribution is categorized to discrete distributions, continuous distributions, and joint distributions (Marray, 1990). 
For statistical evaluation of fatigue testing data, it is often assumed that fatigue life in the finite life regime is log-normally distributed. The log-normal distribution which is one of basic continuous distributions provides a lot of benefits to researchers due to its wide applicability to reliability problems, especially in the area of maintainability and to certain fracture problem.

Mathematically, if $Y$ is $\log$-normally distributed, then $\log Y$ is normally distributed. If a variable can be thought as the multiplicative product of many small independent factors, it is able to be regarded as log-normally distributed.

A probability density function (pdf) serves to represent a probability distribution in terms of integrals. For continuous random variables, the expression for the log-normal probability density function (pdf) and cumulative distribution function (CDF) are given as follow (Mann, Schafer, and Singpurwalla, 1974).

$\begin{array}{lll}\text { pdf } & f(x)=\frac{1}{x \sigma \sqrt{2 \pi}} e^{-\left(-\left[\frac{\ln (x)-\mu}{\sigma}\right]^{2} / 2\right)} & 0 \leq x \leq+\infty \\ \text { CDF } & F(x)=\frac{1}{2}+\frac{1}{2} \operatorname{erf}\left[\frac{\ln (x)-\mu}{\sigma \sqrt{2}}\right] & 0 \leq x \leq+\infty\end{array}$

where: $x$ is the continuous random variable

$\mu$ is the population mean

$\sigma$ is the population standard deviation
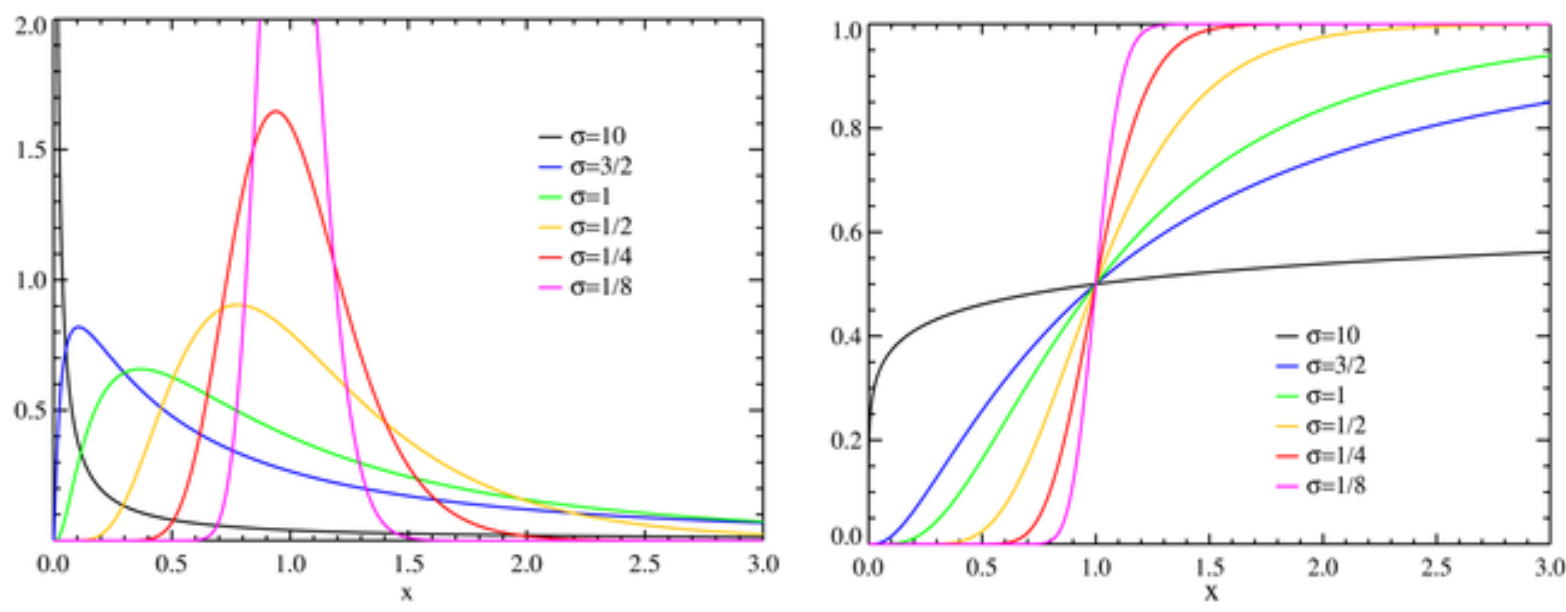

Figure 2.1 Log-normal pdf and CDF (Stahel, Limpert, and Abbt, 2001) 


\subsection{Statistical independent results}

In probability theory, statistical independence means that the occurrence of events makes it neither more nor less probable that the others occur. In fatigue testing, individual specimen is usually tested for the basic research of fatigue strength. The test specimen is usually simple welded and is known the weakest part in terms of the fatigue resistance. For example, a longitudinal fin in a base plate or a base plate welded with longitudinal fins on both sides. Various strain gauges are often implemented in tests and the loading can be multi-axial. Final failure appears in the form of crack growth which is caused by numerous stress ranges (Niemi, Marquis and Poutiainen, 2005). The number of endurance cycles at different stress range level is recorded. Each test is carried out separately and does not affect the outcome each other. In other words, the result from each test is statistically independent of the others.

The above mentioned characteristics are foremost assumptions in the statistical evaluation of fatigue data. The testing data must be match for them in order to truly represent the population. Commonly, the above assumptions are available in practice. There are relative statistical tests which are able to justify their validity. 


\section{FITTING A S-N CURVE}

Simply, the S-N curve is composed of $n$ data points according to stress range and the endurance in cycles. The standard approach in curve fitting is to assume that the parameter on the x-axis is the independent variable and the one on the y-axis is dependent variable.

In the S-N curve, the constant amplitude fatigue limit (CAFL) is defined as the stress range below which failure will not occur. For design purposes, it is assumed that the S-N curve extends down to the CAFL and then turns sharply to become a horizontal line. Typically, the endurance of smooth specimens is around $2 \times 10^{6}$ cycles. For notched specimens, $10^{7}$ cycles is commonly used. Moreover, it is important to note that the test results which lie in region approaching the CAFL should not be used to estimate the best-fit linear S-N curve. These suggestions are from the Best Practice Guide (Schneider and Maddox, 2006).

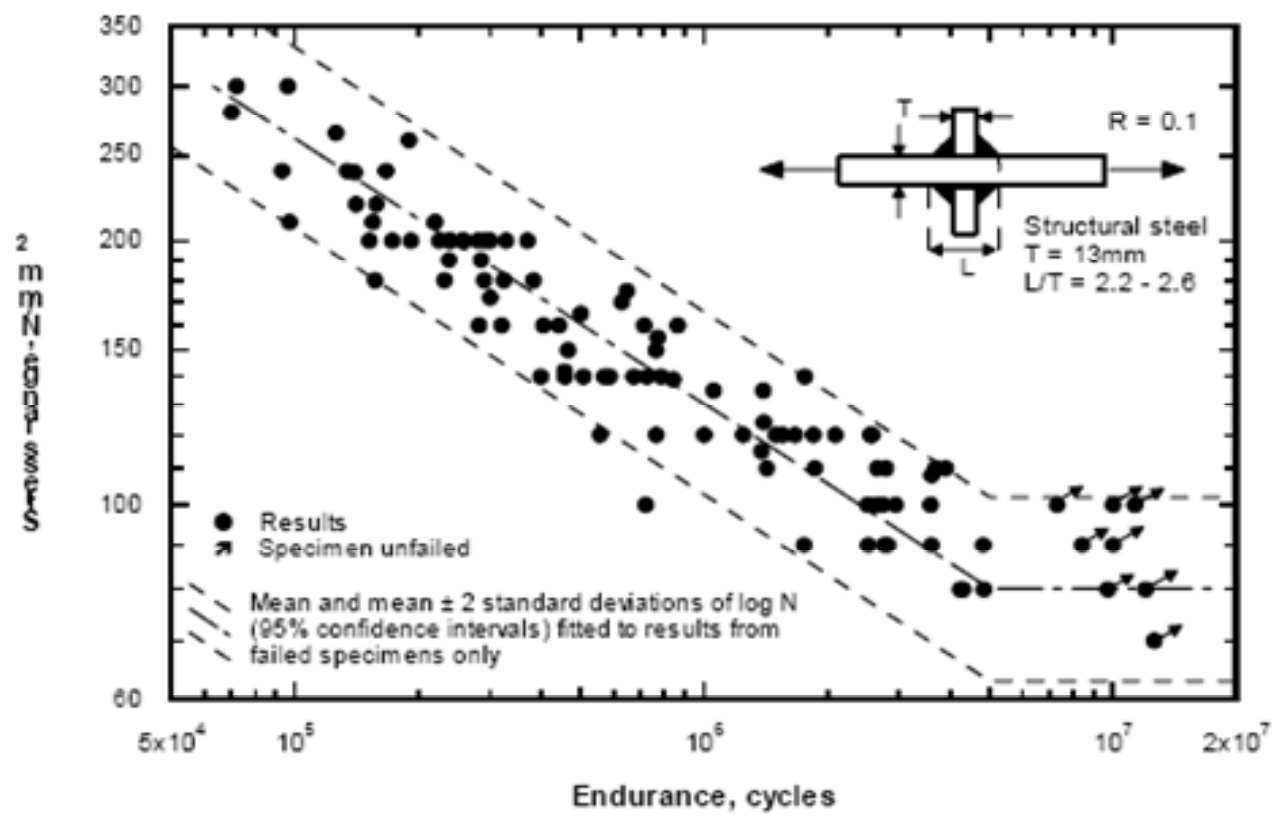

Figure 3.1 Example of S-N curve with data (Maddox, 1993) 


\subsection{Fatigue data}

Statistical evaluation relies extensively on the testing data to make estimations. Hence, the supplied data affects the accuracy of the prediction greatly. The fatigue life data can be separated into two categories: complete data or censored data.

Complete data means that the value of each sample unit is observed or known. In the case of life data analysis, the data set would be composed of the times-to-failure of all units in the sample. For instance, if five samples are tested and all failed, we would have complete information as the time of each failure in the sample. (Reliasoft, 1992)

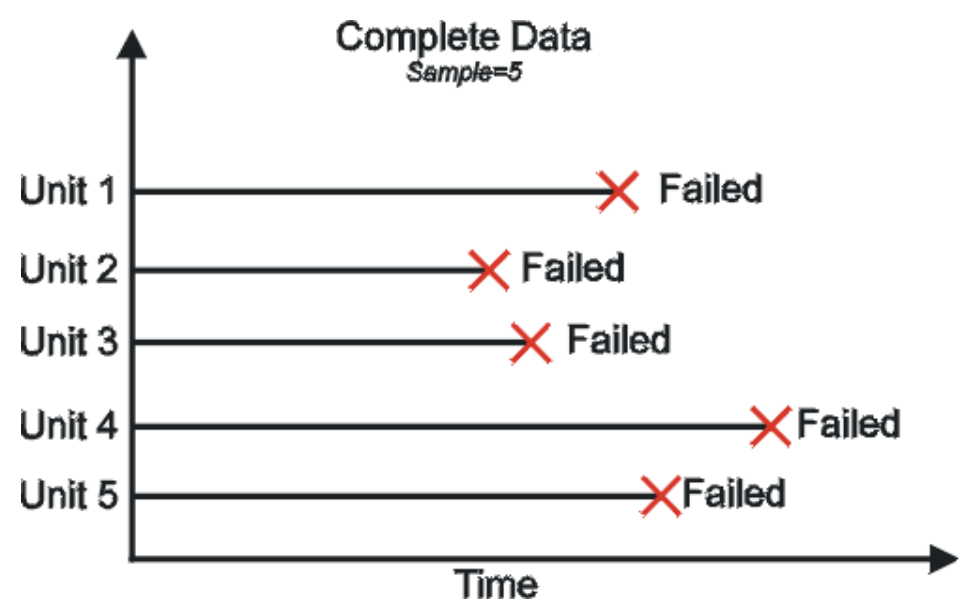

Figure 3.2 Complete data (Reliasoft, 1992)

In fatigue testing, all of the samples may not have failed when the testing finishes. We do not make sure each specimen under fatigue test yielded an exact failure endurance. Therefore, it is possible to obtain results from the specimens or parts of specimens which are unfailed. These unfailed specimen are often termed 'run-outs'. This type of data is commonly called censored data. There are three types of possible censoring schemes, right censored (suspended data), interval censored and left censored.

Right censored is common in the fatigue testing. These data sets are composed of units that did not fail. The failure would yields at some time on the right on the time scale. 


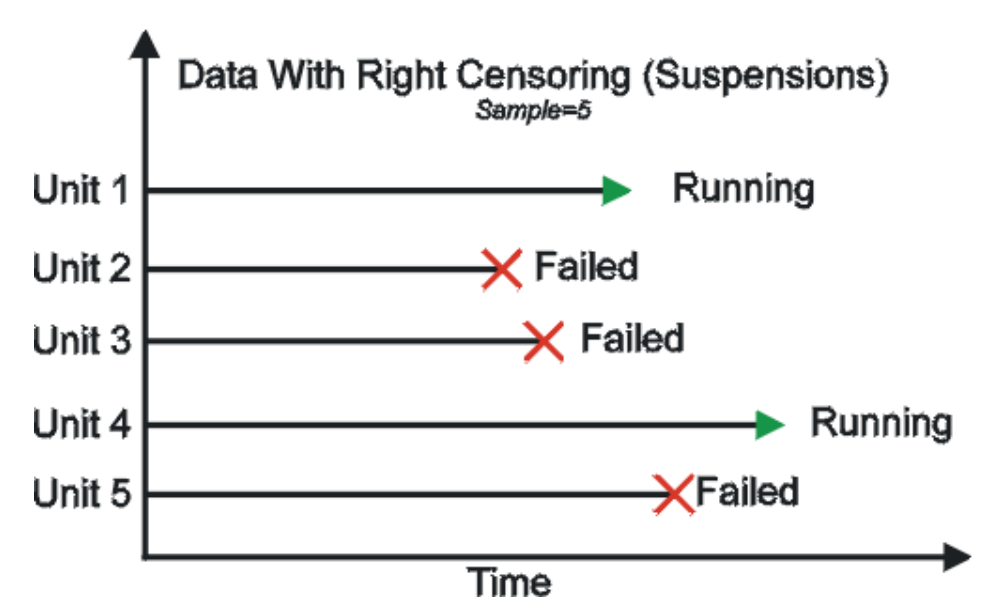

Figure 3.3 Right censoring data (Reliasoft, 1992)

Interval censored data reflects uncertainty as to the exact times the units failed within an interval. We concern about whether the samples are failed in a certain interval of time.

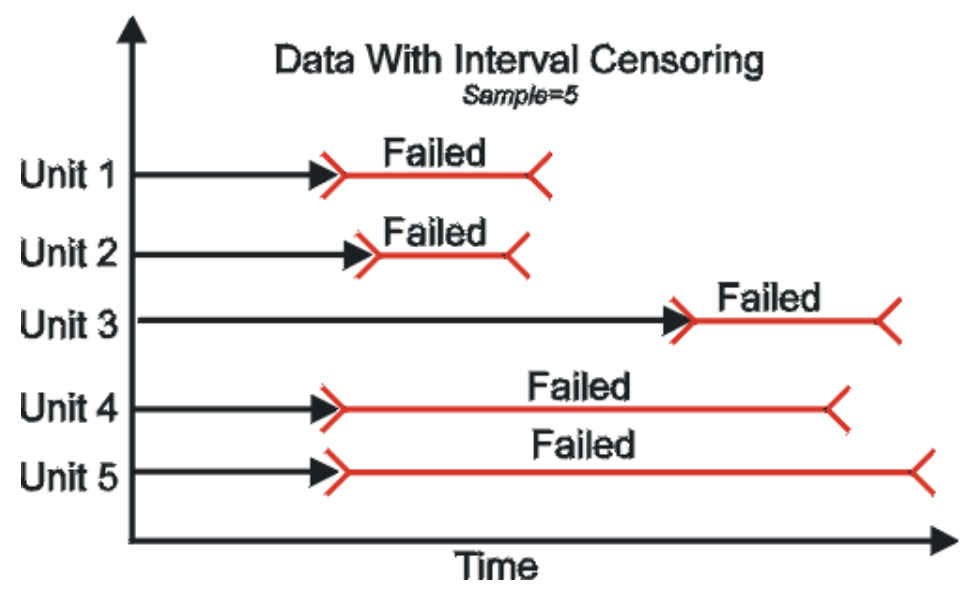

Figure 3.4 Interval censoring data (Reliasoft, 1992)

In left censored data, a failure time is only known before a certain time. For example, we know that a certain unit failed sometime before an interval time.

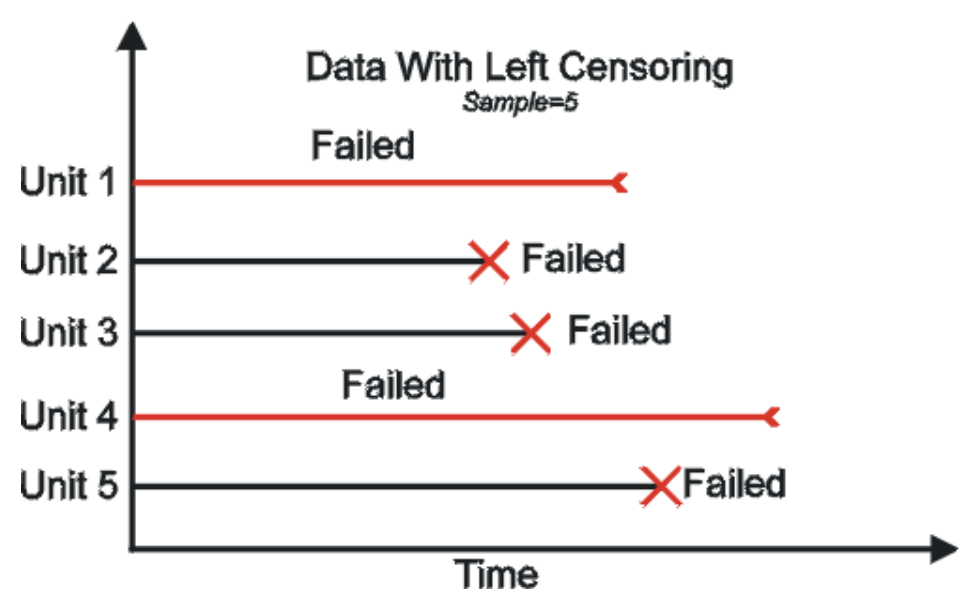

Figure 3.5 Left censoring data (Reliasoft, 1992) 


\subsection{Methods of estimating the S-N curve parameters}

The general problem of finding equations of approximating curves which fit given sets of data is called curve fitting. One of the main purposes of curve fitting is to estimate dependent variable from the other independent variable. The process of estimation is termed as regression. (Spiegel, 1990)

Many methods of statistical evaluation are available to determine best-fitting line and the statistical parameters. Generally, those methods are used to model the univariate data with a specific probability distribution.

Table 3.1 Method of estimating the S-N curve parameters (International Organization for Standardization, 2000)

\begin{tabular}{|c|c|c|c|}
\hline $\begin{array}{c}\text { Method } \\
\text { of } \\
\text { Analysis }\end{array}$ & S-N curve equation & Method and Assumptions & Parameters \\
\hline (A) & $\log N=\log C-m \log S$ & $\begin{array}{c}\text { Mean line bisecting the two } \\
\text { regression lines }\end{array}$ & $C, m,{ }^{\mathrm{s}} \log S$ \\
\hline (B) & $\log N=\log C-m \log S$ & $\begin{array}{c}\text { Linear regression of log } N \text { on log } \mathrm{S} \\
\text { ignoring run-out's }\end{array}$ & $C, m,{ }^{\mathrm{s}} \log S$ \\
\hline (C) & $\log N=\log C-m \log S$ & $\begin{array}{c}\text { Maximum likelihood (includes } \\
\text { run-out's) }\end{array}$ & $C, m,{ }^{\mathrm{s}} \log \mathrm{S}$ \\
\hline (D) & $\log N=\log C-m \log S$ & $\begin{array}{c}\text { Linear regression of log N on log } \mathrm{S} \\
\text { run-out's being included }\end{array}$ & $C, m,{ }^{\mathrm{s}} \log S$ \\
\hline (E) & $N=\frac{A}{S-E}\left[-\frac{(S-E) C}{B}\right.$ & $\begin{array}{c}\text { Multiple non-linear regression } \\
\text { including censored data (run-out's) } \\
\text { Stress response curves: sigmoid } \\
\text { normal }\end{array}$ & $A, B, E$ \\
${ }^{\mathrm{s}} S, F$ \\
\hline
\end{tabular}

It is noted that methods $A$ and $B$ do not take unbroken specimens into account. While other three methods consider the run-outs into account in the analysis and which have a bearing on the final results.

Whether the results from unfailed specimen are used in the statistical analysis of the data depends on the circumstances. But when the fatigue data include either run-outs or 
suspended data, the appropriate statistical analyses become more complicated. Fortunately, many statistical software packages can perform the required calculations according to (International Organization for Standardization, 2000).

\subsection{Least squares regression method}

Considering only the results from truly failed specimens, the ordinary linear regression, called 'least square estimation', is used to estimate the intercept $\log A$ and slope $m$ of the best-fit line through the data. This method attempts to minimize the sum of the squares of the dependent variables between points generated by the function and corresponding points in the data. Note that the implicit requirement of the least squares method to work is the errors in each measurement should be randomly distributed.

Calculate the intercept $\log A$ and slope $m$ for best-fit S-N curve by using least square regression method (Spiegel, 1990):

$Y=A+m X$

$Y=\log N \quad X=\log S$

$\hat{m}=\frac{n \sum x_{i} y_{i}-\sum x_{i} \sum y_{i}}{n \sum x_{i}^{2}-\left(\sum x_{i}\right)^{2}}$

$\hat{A}=\frac{\sum y_{i}}{n}-\hat{m} \frac{\sum x_{i}}{n}$

Linear least square regression is widely used in process modeling because of its effectiveness and completeness. Moreover, linear least squares regression makes very efficient use of the data. Good results can be obtained within relatively small data sets. However, when the data sets include the unusual data points, the estimates will be affected greatly.

Least squares estimation for linear models is notoriously weak to outliers. If the distribution of outliers is skewed, the estimates maybe biased. This problem affects the 
efficiency extremely. When outliers occur in the data, another method such as robust regression is more appropriated to be used.

\subsection{Maximum likelihood regression method}

We suggested a method of curve fitting the probability density function to the test data in a maximum likelihood sense. It has the advantage of considering every valid observation of the sample. This method expressed the probability of the combined events failures and runouts to find the optimum estimates of the sample mean and variance.

Simply, when the fatigue life data satisfied with the assumptions mentioned in section 2 and are neither run-outs nor suspended data, the following maximum likelihood estimators of intercept $\log A$ and slope $m$ are recommended to use (ASTM international, 2004):

The S-N relationship is described by the linear model:

$$
Y=A+m X
$$

$Y=\log N$

$$
X=\log S
$$

$$
\begin{aligned}
& \hat{A}=\bar{Y}-\hat{m} \bar{X} \\
& \hat{m}=\frac{\sum_{i=1}^{k}\left(X_{i}-\bar{X}\right)\left(Y_{i}-\bar{Y}\right)}{\sum_{i=1}^{k}\left(X_{i}-\bar{X}\right)^{2}}
\end{aligned}
$$

Maximum likelihood Method can be developed for a large variety of estimation situations. As the sample size increases, it offers minimum variance unbiased estimators. The approximate normal distributions and sample variances can be used to generate confidence bounds and hypothesis tests for the parameters. However, the estimates might be heavily biased for small samples. Additionally, the calculation is laborious without computer assistant. 


\subsection{Sample size and population}

In practice we are interested in drawing valid conclusions about a large group of individuals or objects. Instead of examining the entire group, called the population, which may be difficult to do, one may arrive at the idea of examining only a small part of this population, which is called a sample (Spiegel, 1990).

In fatigue testing, we face a finite population which is sampled with replacement. "How many measurements should be included in the sample?” It is one of the most frequent questions which need to be considered for statistical evaluation. Sample size determines the precision of that estimate. Larger sample size gives smaller error bounds of estimation. In order to determine the sample size we need to consider a series of factors such as cost of sampling, variability of the population, the precision of the final estimation. Fortunately, the prior information derived form previous study or research can be used to reduce the sample size.

Commonly, there is no correct answer without additional information or specific assumptions. But the following steps can help us basic idea how to solve the problem. First, determine what we are trying to estimate and how precise we want. Second, find some equations that connect the desired precision of the estimate with the sample size. Third, get unknown properties in the equations. Final, review the final sample size to make sure it is acceptable.

Actually, the researcher should make sampling decisions based on the data. The basic sample size determinations can be categorized according to the type of continuous data and categorical data. Continuous data is measured on a scale and can be meaningfully subdivided into finer and finer increments. Categorical variables represent types of data which may be divided into groups. Before proceeding with sample size calculations, assuming continuous data, it is important to determine if a categorical variable will play a 
primary role in data analysis. For fatigue analysis, the fatigue data are continuous data because they are continuous in stress range.

In this work, I study this question: How many results are necessary to validate a selected S-N curve? The assumption is that the slope and standard deviation of the mean S-N curve of the new test results are the same as those parameters of the selected S-N curve. Then the null hypothesis that the new test results belong to the same population as the main data base is made (Schneider and Maddox, 2006)

$\overline{\log A_{\text {test }}} \geq \overline{\log A}+\frac{Z_{p \%} \sigma}{\sqrt{n}}$

where: $\overline{\log A_{\text {test }}}$ is the mean logarithm of interception from new tests

$\overline{\log A}$ is the logarithm of the interception from the mean for the selected S-N curve

$Z_{p \%}$ is standard normal probability for a probability

The above equation can be transformed into following form to determine the sample size:

$$
n \geq z_{\alpha}^{2}\left(\frac{\sigma^{2}}{\delta^{2}}\right)
$$

where: $\sigma$ is the population standard deviation

$z_{\alpha}$ is the ordinate on the normal curve corresponding to $\alpha$

$\delta$ is the specified precision of the estimate. For instance, $\delta=\overline{\log A_{\text {test }}}-\overline{\log A}$.

It is indicates that the essential thing of determination sample size for fatigue testing is to determine a sample size that is large enough to guarantee the risk. In other hand, sample along with high quality data collection efforts will result in more reliable, valid, and generalizable results. It is able to do resource saving greatly. 


\section{ESTABLISHING A DESIGN CURVE}

\subsection{Mean, characteristic and design values}

Generally, a structure can be damaged in various ways. Calculation of a single value such as strength value for a structure does not ensure that the structure itself will not fail. Numbers of values are needed to be obtained from the alternated failure modes. Then the mean value is often to be calculated to report central tendency. The mean value is not appropriate for describing skewed distributions. Fortunately, samples from fatigue testing are normal distributed.

In practice, there are some load events which are not known in advance. Therefore, designer often uses limit state design to avoid failures due to unforeseen events. A limit state is a condition beyond which a structure is less than suitable to perform the required function. Fundamental ideas of limit state design include statistically based definitions of characteristic strength, characteristic loads and characteristic stress. By using those characteristic values associated with partial safety factor, the design values are able to be calculated. (Niemi, Marquis and Poutiainen, 2005).

Commonly, on the load side, the characteristic value is determined that it is larger than the mean value by two times the standard deviations. The design value can be calculated by using the characteristic value multiply the partial safety factor for fatigue actions. On the resistance side, the characteristic value is defined that the mean value subtracted twice standard deviation. The design value can be obtained by using the characteristic value divided by the partial safety factor for fatigue resistance. Moreover, using the twice standard deviations is based on the $95 \%$ probability of survival to reach a reliability of $75 \%$.

The figure 4.1 indicates that, on resistance side, the mean value and characteristic value according to $95 \%$ probability of survival. 


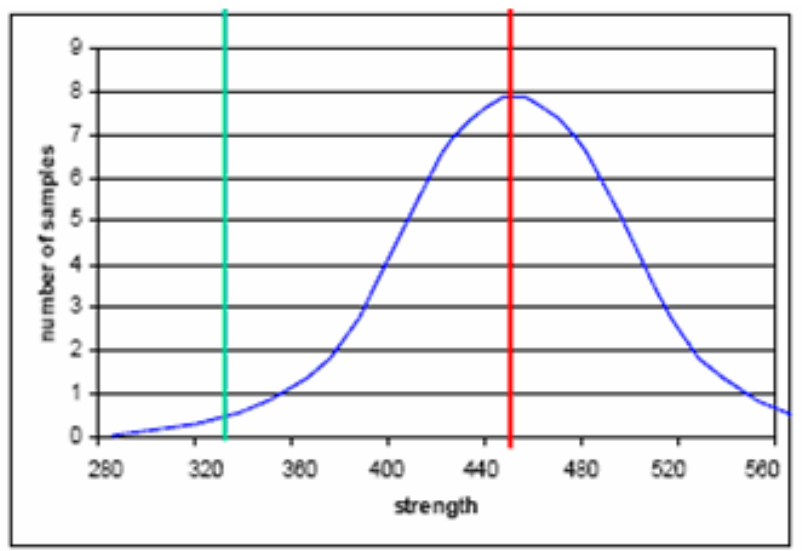

Figure 4.1 Mean and characteristic strength

In most cases for normal fabrication quality and regular inspections, the partial safety factor is selected to be 1 . Therefore, a characteristic or design curve is established by adopting characteristic values that lay a certain number of standard deviations below the mean S-N curve as the figure 3.1. Fatigue life verification of fail safe structures depends largely on the design parameters of a structure. Three different methods have been developed to determine the design curve.

\subsection{Confidence limits}

When we take a random sample from a population to approximate the mean of the population, we concern about how well the sample statistically estimates the population value. The confidence limits, also called as confidence interval, which provides a range of values which is likely to contain the population parameter of interest. Confidence intervals with a confidence level mean that if the population is sampled on numerous occasions and interval estimates are made on each occasion, the resulting intervals would bracket the true population parameter in approximately the certain percentage of the confidence level (NIST/SEMATECH, 2003). For example, a 95\% confidence interval means that if many samples are collected and the confidence interval computed, in the long run about $95 \%$ of these intervals would contain the true mean. 
A fatigue design or characteristic curve is established by adopting characteristic values that lay a certain number of standard deviations below the mean S-N curve. It has to be accepted that only a small part of the samples can be tested in practice. It is normally accepted that the sample mean of normal distribution is characterized by the Student's $t$ distribution and the sample variance is characterized by the chi-square distribution.

When the confidence is applied to the sample mean and variance, the sample mean and variance will be limited without the influence from number of tests. These values with confidence are used as the mean value respective the variance for the whole population. In principle, the characteristic values are values at a $\alpha=95 \%$ survival probability associated to confidence interval of the mean and the standard deviation:

$x_{k}=\bar{x}-k_{c} \cdot s$

The factor $k_{c}$ considers the effects of variance of data and deviation evaluated. It corresponds to the minimum value of the mean confidence interval and maximum value of the variance confidence interval. Taking into account the probability distribution of the mean corresponds to student $t$-distribution and the variance corresponds to Chi-square distribution, it can be calculated by using following equation (Hobbacher, 2005):

$$
k_{c}=\frac{t_{(p, n-1)}}{\sqrt{n}}+\Phi_{(\text {alpa })}^{-1} \sqrt{\frac{n-1}{\chi_{\left(\frac{1+\beta}{2}, n-1\right)}^{2}}}
$$

where: $t$ value of the two sided t-distribution

$\Phi$ distribution function of the Gaussian normal distribution probability

$\chi^{2}$ Chi-square for a probability at $n-1$ degrees of freedom

Normally we use 95\% confidence level to analysis the test results. The following figure shows the typical confidence limits. 


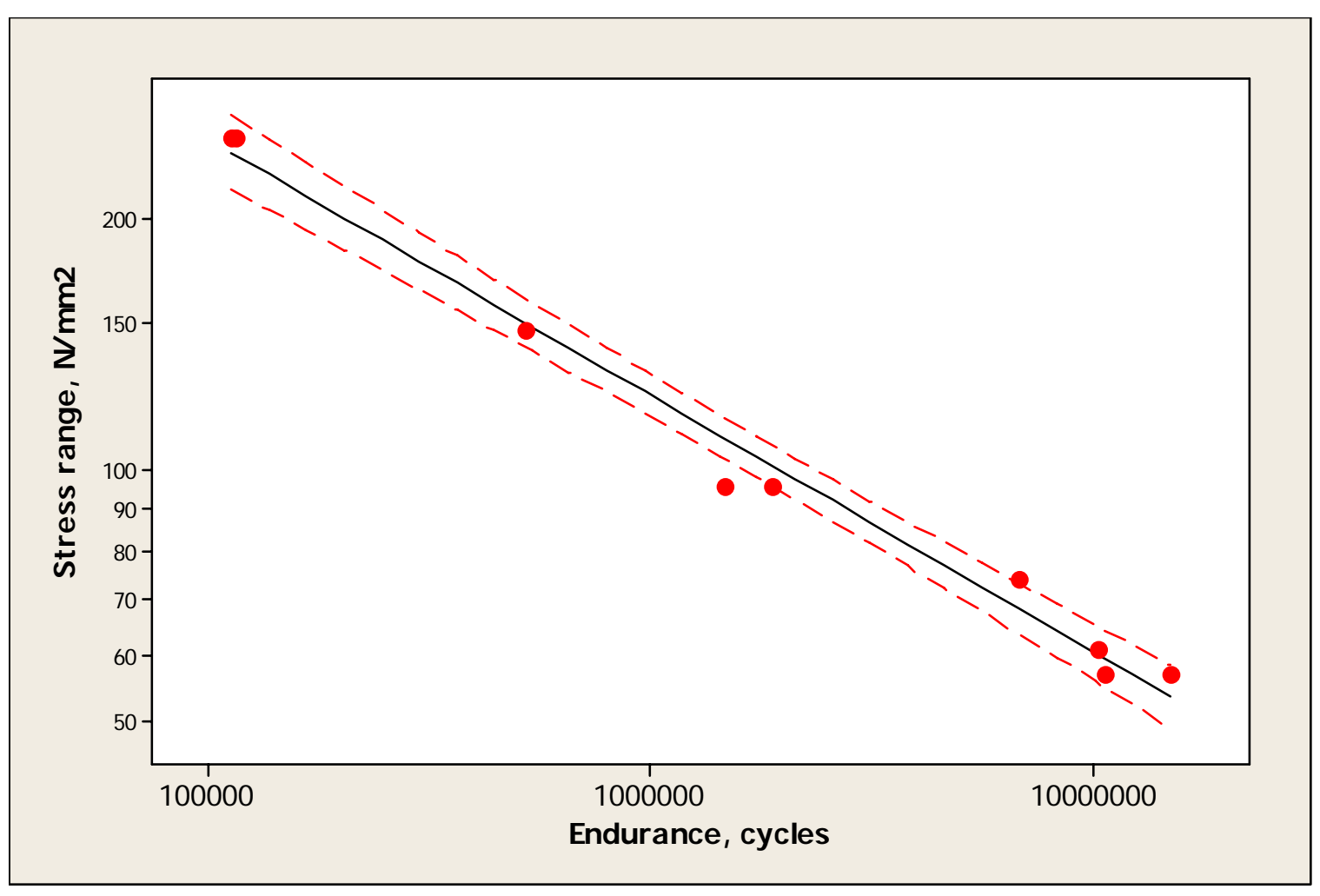

Figure 4.2 Regression line and design curve based on $95 \%$ confidence limits

\subsection{Prediction limits}

The prediction limit describes the probability that an individual point will be above or below a certain value. A two-sided prediction limit sets an interval for the data while a one-sided prediction limit describes the probability that a point will exceed a certain value.

For the normally distributed fatigue data sets, the prediction limits at stress range $S$ can be expressed explicitly in the form (Schneider and Maddox, 2006):

$$
\log N_{P \%}^{ \pm}=(\log A+m \log S) \pm t \hat{\sigma} \sqrt{1+\frac{1}{n}+\frac{(\log S-\overline{\log S})^{2}}{\sum_{i=1}^{n}\left(\log S_{i}-\overline{\log S}\right)^{2}}}
$$

where: $\log \mathrm{A}$ and $m$ are the coefficients of the regression line through the $n$ data points $\overline{\log S}$ is the mean of the $n$ values of $\log S_{i}$ 
$t \quad$ is the appropriate percentage point of Students $t$ distribution

$\hat{\sigma} \quad$ is the best estimate of the variance of the data about the regression line,

$f \quad$ is degrees of freedom, $f=n-2$ in case where the two coefficients of the regression line have been estimated from the data

When the slope is chosen to take a fixed value, the expression under the square roots is exactly equal to one. It is recommended to use when the sample size is less than ten. But when the sample sizes are smaller than 4 , the following method of tolerance limits is regard as a more conservative design curve compared with prediction limits.

\subsection{Tolerance limits}

According to the "Best Practice Guide on Statistical Analysis of Fatigue Data” (Schneider and Maddox, 2006), the tolerance limits yield a more conservative design curve. The disadvantages of tolerance limits also could be ignored. They are inherently more complicated and harder to implement. They are more sensitive to deviations from the assumed normality.

Generally, the tolerance limits are defined by lower and upper tolerance limits which are calculated from a series of results. For fatigue testing, the lower one-sided $P \%$ tolerance limit takes general form:

$\log N_{P \%}^{-}=\hat{\mu}-k s$

where: $\hat{\mu}$ is an estimate of the mean $\log$ of the endurance at stress $S$, based on $n$ observations

$S$ is an estimate of the standard deviation of the log of the endurance at stress $\mathrm{S}$, based on degree of freedom

$k$ is a one-sided tolerance limit factor

Estimating lower confidence limits of the form $\hat{\mu}-k s$ on the prediction limit is available to avoid the sampling uncertainties. This statement means that at least a proportion 
$P \%$ of the population is greater than $\hat{\mu}-k s$ with confidence $\gamma \%$.

For fatigue analysis, if the slope of the regression line is estimated from the data, tolerance limit factors for the normal distribution are not easy to obtain. It needs the evaluation of both $P \%$ percentage points of the normal distribution and the $\gamma \%$ percentage points of the noncentral $t$ distribution. Based on the handbook of statistical tables (Owen, 1962), $k$ is determined by:

$\operatorname{Pr}\left\{\left(\right.\right.$ noncenral $t$ with $\left.\left.\delta=K_{P} \sqrt{n}\right) \leq k \sqrt{n}\right\}=\gamma$

where: the noncentral $t$ has $f$ degrees of freedom

$$
K_{P} \text { is defined by } \frac{1}{\sqrt{2 \pi}} \int_{-\infty}^{K_{p}} \exp \left(-x^{2} / 2\right) d x=P
$$

The main trouble of calculation is to obtain the critical values $t_{0}$ of the noncentral $t$-distribution, because its calculation involved several parameters such as $\eta, \delta, \lambda$. Tables of factors for computing critical values of the noncentral $t$-distribution in (Owen, 1962) are used to calculate value of $\lambda$ according to $\eta, \delta$. After obtaining corresponding value $\lambda$, critical value $t_{0}$ is obtained by the formula:

$$
t_{0}=\frac{\delta+\lambda\left(1+\frac{\delta^{2}}{2 f}-\frac{\lambda^{2}}{2 f}\right)}{1-\left(\lambda^{2} / 2 f\right)}
$$

Then $k$ value can be obtained by equation:

$$
k=\frac{t_{0}}{\sqrt{n}}
$$

For the case of degree of freedom $f=n-2$, the relative laborious calculation has been done in this thesis. The common used $k$ values are able to be find from the table of one-sided tolerance limit factor in appendix XI

While, if the slope is fixed, the tolerance factors for several cases which are interested in 
fatigue analysis are offered in appendix X from (Schneider and Maddox, 2006).

\subsection{Comparison}

Typically, confidence limits are based on sample mean and sample standard deviations. Therefore, it is usually recommended to use when we have larger sample sizes. Prediction intervals are especially powerful because they can predict a future compliance point. Its requirement of sample size is low. The tolerance limits is a way to determine a range that will contain a certain percentage of the population. It is recommended to be used as a means of justifying design curves that are based on small samples, especially for critical applications.

Figure 4.3 is the design curve based on 95\% confidence limits and prediction limits for the same regression line. It indicates prediction limits are more conservative than confidence limits.

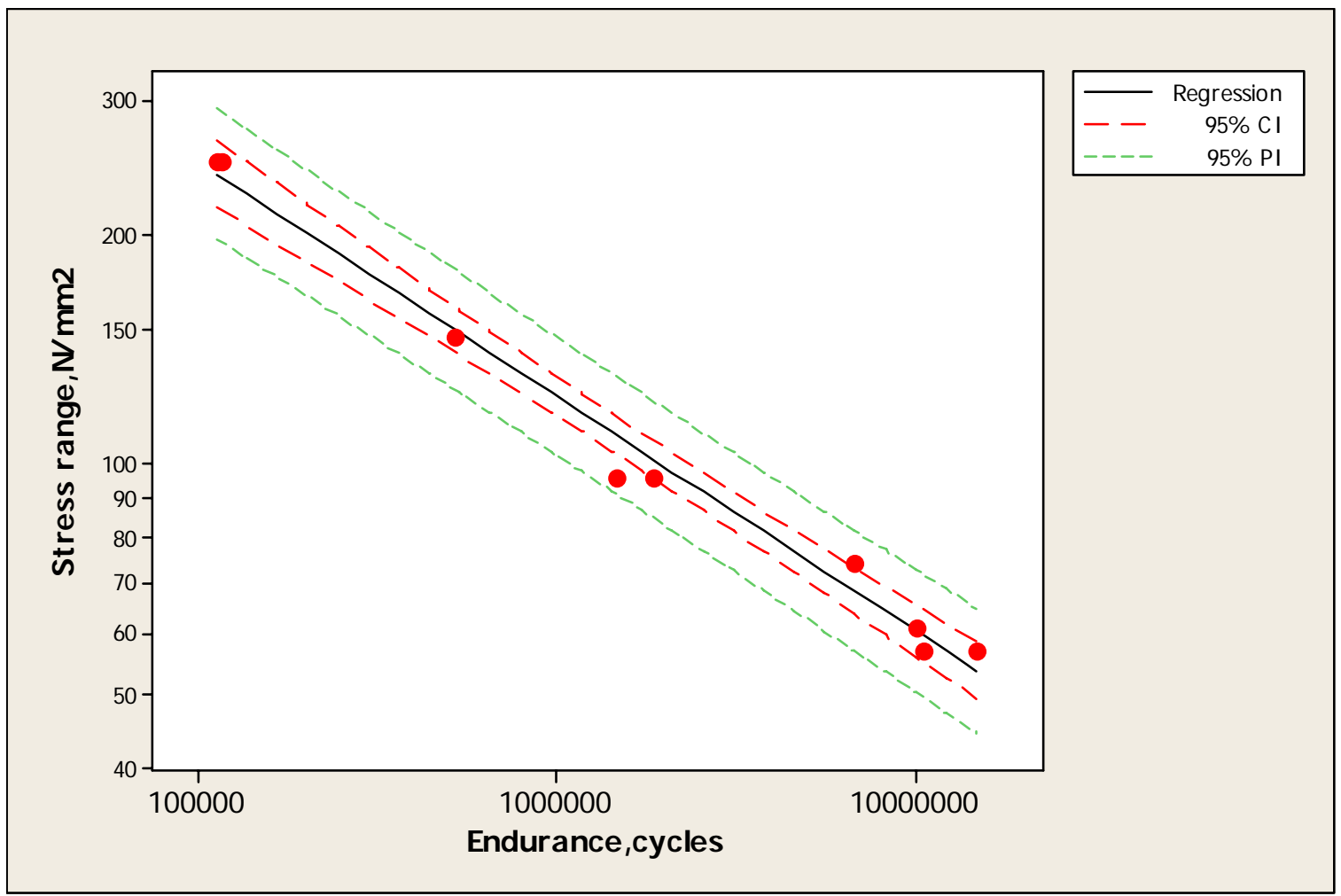

Figure 4.3 Design S-N curve based on confidence and prediction limits 


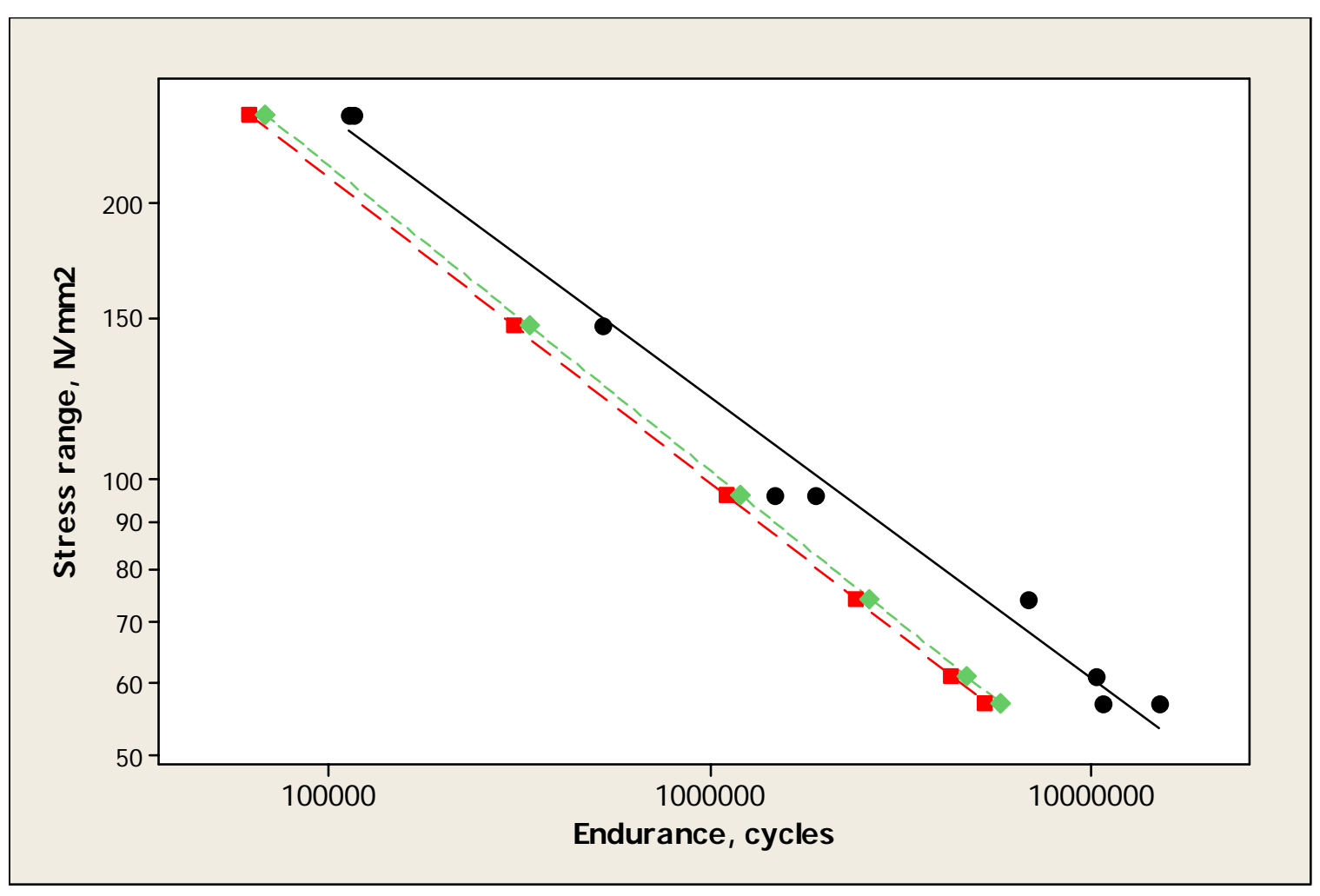

Figure 4.4 Design S-N curve based on prediction and tolerance limits

The figure 4.4 shows the comparison of the results from data sheets 10 and 12 . The green and red line respectively expresses the design curves based on prediction and tolerance limits. It is obvious that the tolerance limits offers a lower design curve. It proves that tolerance limits are more conservative than prediction limits. 


\section{VERIFICATION OF THE STATISTICAL EQUIVALENCE FOR TWO S-N CURVES}

In manufacturing field, when the manufacturing process has been changed or new processing technology is used, we want to determine whether a significant change has been produced. In statistical evaluation of fatigue data, this problem is transformed to justify the significant difference between two sets of S-N data or whether two sets of S-N data from the same population.

A null hypothesis is often used to check this problem. In statistics, a null hypothesis is a hypothesis set up to be nullified or refuted in order to support an alternative hypothesis. This is the basis for regarding the null hypotheses as plausible, and for rejecting the alternative hypothesis that two data sets of test results belong to different populations. In this thesis, it is assumed that both sets of S-N data are exact data even though this approach can be extended to the case of censored data in principle. (Spiegel, 1990)

The limitation of this a null hypothesis is that it is only useful if it is possible to calculate the probability of observing a data set with particular parameters form it. It is much harder to be precise about how probable the data would be if the alternative hypothesis is true. The theory underlying the idea of a null hypothesis is closely associated with the frequency theory of probability. A failure to reject the null hypothesis is meaningful only in relation to an arbitrarily large population from which the observed sample is supposed to be drawn.

\subsection{Tests performed to an S-N curve}

When we consider the statistical equivalence of the two S-N curves, the residual standard deviation, the intercepts and slopes of the two S-N curves are need to be taken into account. These formulas have been given by (Schneider and Maddox, 2006): 
Test the residual standard deviation

Null hypothesis: two sets of results belong to populations having the same standard deviation.

$\frac{\hat{\sigma}_{1}^{2}}{\hat{\sigma}_{2}^{2}} \leq F_{f_{1}}^{f_{2}}$

where: $F_{f_{1}}^{f_{2}}$ is the $P \%$ percentage point of the $F$ distribution

$\hat{\sigma}^{2}$ is the best estimated variances. $\hat{\sigma}_{1}^{2}>\hat{\sigma}_{2}^{2}$

Test the intercepts of the two S-N curves

Null hypothesis: two sets of results belong to populations having the same intercept.

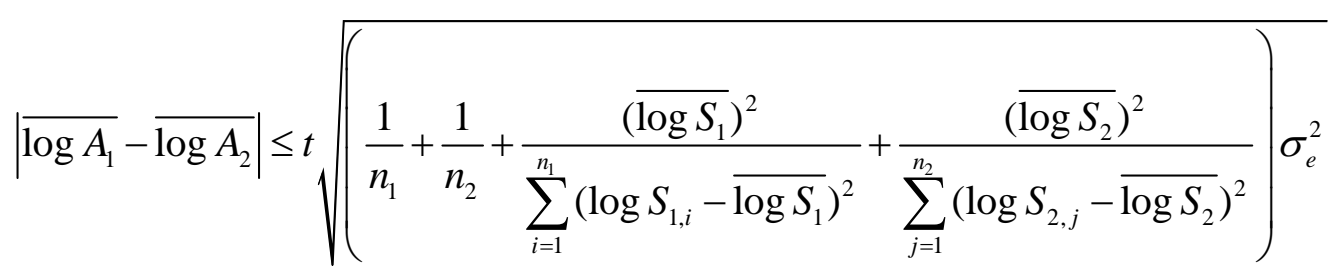

where: $\overline{\log A_{1}}, \overline{\log A_{2}}$ are the estimated intercepts of the regression lines of two data sets

$t$ is the appropriate two-sided percentage point of Student'st distribution, with degrees of freedom $n_{1}+n_{2}-4$

$\sigma_{e}$ is an estimate of the common variance of the two samples, $\sigma_{e}^{2}=\frac{f_{1} \hat{\sigma}_{1}^{2}+f_{2} \hat{\sigma}_{2}^{2}}{f_{1}+f_{2}}$

Test the slopes of the two S-N curves

Null hypothesis: two sets of results belong to populations having the same slope.

$$
\left|m_{1}-m_{2}\right| \leq t \sqrt{\left(\frac{1}{\sum_{i=1}^{n_{1}}\left(\log S_{1, i}-\overline{\log S_{1}}\right)^{2}}+\frac{1}{\sum_{j=1}^{n_{2}}\left(\log S_{2, j}-\overline{\log S_{2}}\right)^{2}}\right) \sigma_{e}^{2}}
$$

where: $m_{1}, m_{2}$ are the estimated slopes of the regression lines of the two data sets

In this case, the numbers of degree of freedom should be equal to $n-2$, because two coefficients of intercept and slope have been estimated to obtain the S-N curves. At the same time, the significance level of the null hypothesis should be noted. For instance, each of the three null hypotheses is tested at 5\% significance level; the combination of the 
probability is $15 \%$. Therefore the guild (Schneider and Maddox, 2006) is recommended choosing $1.7 \%$ significance level for each individual test in order to get a $5 \%$ significance level of the composite hypothesis.

Here, I need to emphasize the definition of the significance level. In this hypothesis testing, the significance level of a test is the maximum probability, assuming the null hypothesis, that the statistic would be observed. Therefore, the significance level is the probability that the null hypothesis will be rejected in error when it is true. That means when we use significance level $1.7 \%$ instead of $5 \%$, we have more opportunities to accept the null hypothesis.

For example, test whether two sets of results belong to populations having the same slope. The null hypothesis at a significance level $\alpha \%$ is accepted if the equation (5.3) is satisfied. When we reduce the significance level, the critical region is increased due to growing value of $t$. However, a smaller significance level is possible to give greater risks of failing to reject a false null significance. 


\section{VERIFICATION OF WEIBULL DISTRIBUTED DATA}

\subsection{Weibull probability graph}

Weibull probability plot is a graphical technique for assessing whether or not a data set follows the Weibull distribution. The data are plotted against a theoretical distribution in such a way that the points should form approximately a straight line. In this plot, the departures from this straight line indicate departures from the Weibull distribution.

The Weibull distribution has a relatively simple distribution form, while, the shape parameter allows it to assume a wide variety of shapes. This combination of simplicity and flexibility in the shape of the Weibull distribution has made it to be an effective distributional model in reliability applications. Depending on the parameters' values, the Weibull distribution can approximate an exponential, a normal or a skewed distribution. The standard form of any distribution is the form that has location parameter zero and scale parameter one. Therefore, the Weibull plot is a graphical technique for determining if data sets from a population that would logically be fitted by a 2-parameter Weibull distribution. The following graphs show the Weibull distribution function with shape parameters: 0.5, 1.0, 2.0 and 5.0. (Mann, Schafer and Singpurwalla, 1974)
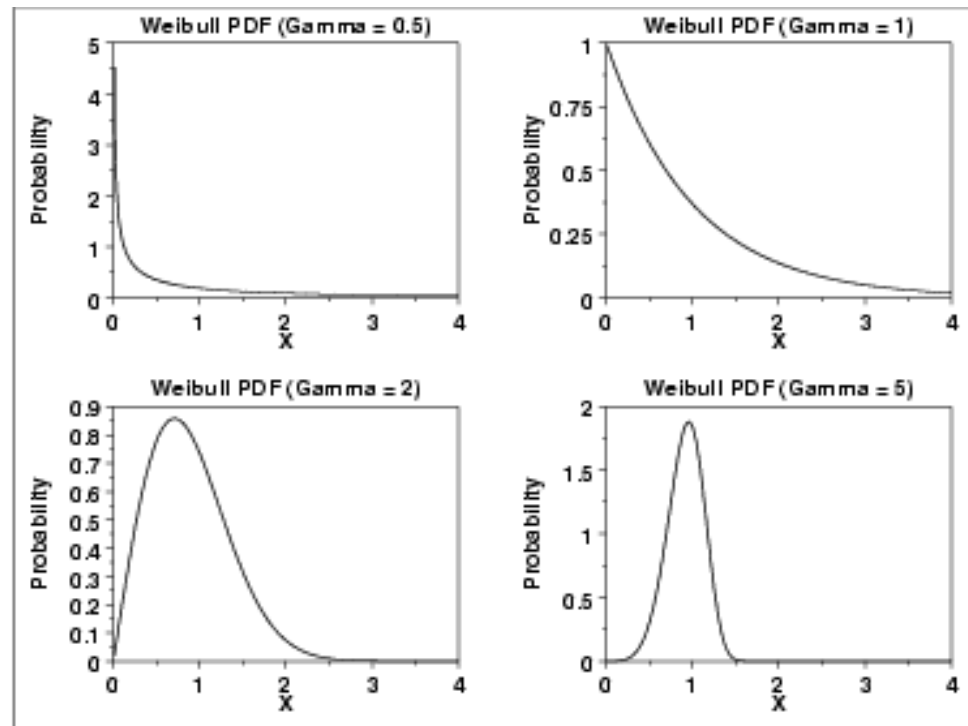

Figure 6.1 Weibull distribution function with different shape parameters (NIST/SEMATECH, 2003) 
Generally, the Weibull shape parameter indicates whether the failure rate is increasing, constant or decreasing. The value of shape parameter $\beta<1.0$ indicates that a decreasing failure rate. The $\beta=1.0$ indicates a constant failure rate and $\beta>1.0$ indicates an increasing failure rate.

The weibull distribution density function is given by (Mann, Schafer and Singpurwalla, 1974):

$$
f(x)=\frac{\beta}{\eta}\left(\frac{x-\gamma}{\eta}\right)^{\beta-1} e^{-\left(\frac{x-\gamma}{\eta}\right)^{\beta}}, \beta>0, \eta>0, x>\gamma \geq 0
$$

The cumulative Weibull distribution function is given by (Mann, Schafer and Singpurwalla, 1974):

$$
F(x)=1-e^{-\left(\frac{x-\gamma}{\eta}\right)^{\beta}}
$$

where: $\beta$ is the shape parameter, $\eta$ is the scale parameter, and $\gamma$ is the location parameter.

Letting $\gamma=0$, we change the form of the cumulative Weibull distribution function equation:

$$
\ln \left(\ln \left[\frac{1}{1-F(x)}\right]\right)=\beta \ln x-\beta \ln \eta
$$

Formula for reliability assuming a Weibull distribution is given in (Dorner, 1999):

$$
R(t)=e^{-\left(\frac{x}{\eta}\right)^{\beta}}
$$

Then the Weibull probably graph can be plotted corresponding to above equations. The probability plot is formed by: Vertical axis: Ordered reliability of responsible values and Horizontal axis: Order endurance cycles. It is possible to visualize the reliability of each design for multiple cycle values. 


\subsection{Determine the shape parameter and scale parameter}

For distribution with shape parameters, the shape parameters are necessary to generate the Weibull probability graph. For Weibull distribution which has only single shape parameter, the shape parameter can be estimated by probability plot correlation correlation coefficient plot (PPCC). PPCC is a graphical technique for identifying the shape parameter for a distribution family. It is suitable for Weibull distribution perfectly.

The PPCC plot is used first to find a good choice for estimating the shape parameter of Weibull distribution. Then find estimates of the location and scale parameter. First of all, estimate the $F\left(x_{i}\right)$ from following methods. Commonly, we choose the median rank.

Table 6.1 Methods of estimating $F\left(x_{i}\right)$ (Al-Fawzan, 2000)

\begin{tabular}{|c|c|}
\hline Method & $F\left(x_{i}\right)$ \\
\hline Mean Rank & $\frac{i}{n+1}$ \\
\hline Median Rank & $\frac{i-0.3}{n+0.4}$ \\
\hline Symmetrical CDF & $\frac{i-0.5}{n}$ \\
\hline
\end{tabular}

Then use the least squares method to estimate the shape parameter $\beta$ in equation 6.3. When we perform the linear regression, we will find the estimate for Weibull $\beta$ parameter comes directly form the slope of the line. The estimate for the scale parameter $\eta$ must be calculated as follows:

$\eta=e^{-\left(\frac{A}{\beta}\right)}$

where: $A$ is estimated for the linear regression $Y=A+\beta X$ 


\subsection{A goodness of fit test: Anderson-Darling}

When we assume the fatigue data follow a specific distribution, e.g. Weibull distribution, it takes a serious risk. If our assumption is wrong, the results obtained from research could be invalid and useless.

According to document: Anderson-Darling: A Goodness of Fit Test for Small Samples Assumptions from DoD Reliability Analysis Center (Volume 10, Number 5), there are two main approaches to checking distribution assumptions. One involves empirical procedures and is based on the intuitive and graphical properties of the distribution. Another one is more formal. It is the goodness of fit test. The results form latter approach is more reliable than those form the empirical procedure. At the same time, Goodness of fit test is essentially based on either of two distribution elements: the cumulative distribution function (CDF) or the probability density function (pdf). The Anderson-Darling (AD) test which use CDF is suitable for small samples. Moreover, this AD test is also used in the Minitab software.

In $\mathrm{AD}$ test, we assume a pre-specified distribution to estimate the distribution parameters. This process generates a distribution hypothesis. When the assumed distribution is correct, the theoretical CDF closely follows the empirical step function CDF, as conceptually illustrated in Fatigue 6.2. The data are given as an ordered sample and the assumed distribution has a CDF. We will compare the theoretical and empirical results. If they agree, the data supports the assumed distribution.

The following equation is used to calculate the $\mathrm{AD}$ value for fitting Weibull distribution:

$$
A D=\sum_{i=1}^{n} \frac{1-2 i}{n}\left\{\ln \left(1-\exp \left(-Z_{(i)}\right)\right)-Z_{(n+1-i)}\right\}-n
$$

where: $Z_{(i)}=\left[x_{(i)} / \eta\right]^{\beta}$

$$
A D^{*}=(1+0.2 / \sqrt{n}) A D \quad \text { (Corresponding to the estimation) }
$$


The OSL is given:

$$
O S L=1 /\left\{1+\exp \left[-0.1+1.24 \ln \left(A D^{*}\right)+4.48\left(A D^{*}\right)\right]\right\}
$$

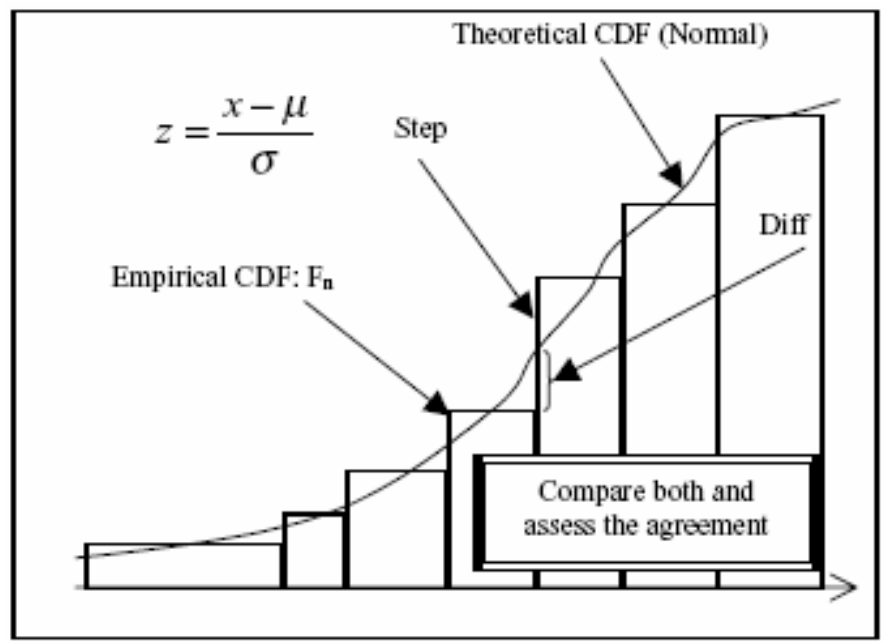

Figure 6.2 Distance goodness of fit test conceptual approach

Then the observed significance level (OSL) probability is used for testing the Weibull assumption. If this p-value is less then 0.05 , Weibull assumption is rejected and the error committed is less than 5\%. In other hand, the null hypothesis is accepted. 


\section{DETERMINE THE IMPROVEMENT FROM POST-WELD TREATMET}

7.1 post-weld treatment

The dominant intention in fatigue design of load-carrying structures in mechanical engineering is to prevent fatigue failure. During cyclic load, it has been turned out that the weakest point in fabricated structures is normally the welded joints. In the weld area, it presents high stress concentration and high tensile residual stress.

The post-weld treatment is a good method to improve fatigue resistance of welded joints. The principles of this method are: (a) to modify the stress distribution near the weld to produce beneficial compressive residual stress. (b) to modify the local geometry of the weld toe to eliminate the initial defects and decrease the local stress concentration.

For the most improvement techniques such as needle peening, hammer peening and ultrasonic impact treatment, the magnitude of the improvement depends on the joint severity and base material strength. The benefit for steel can only be achieved in design class FAT 90 or lower in the IIW notation for S-N curves according to (Lihavainen Marquis and Statnikov, 1990).

\subsection{Statistical analysis of the test data}

The null hypothesis also can be used to analyze this problem to justify the significant difference between the data sets from as-weld and the data sets form post-weld. But it is much harder to be precise about how probable the data would be if the alternative hypothesis were true as we mentioned in section 5 . Therefore, the fatigue class (FAT) is calculated to compare the difference of them in order to derive the degree of improvement. 
The FAT indicates the characteristic stress range, which gives a fatigue life of two million cycles at $95 \%$ survival probability. The method of statistical analysis is developed within the International Institute of Welding (Niemi, Marquis and Poutiainen, 2005):

$\Delta \sigma_{i}^{m} \cdot N_{i}=C_{i}=F A T^{m} \cdot 2000000$

Mean fatigue capacity $\log C_{50 \%}=\frac{\sum \log C_{i}}{n}$

Standard deviation $\quad s=\sqrt{\frac{\sum\left(\log C_{i}-\log C_{50 \%}\right)^{2}}{n-1}}$

$\log C_{95 \%}=\log C_{50 \%}-s\left(1.64+\frac{1.15}{\sqrt{n}}\right)$

Characteristic Fatigue capacity $F A T_{95 \%}=\sqrt[m]{\frac{C_{95 \%}}{2000000}}$

Note that: due to the relatively small sample size and the convenience of evaluation, the slope is choose to be 3 for welded steel joint which the fatigue life is dominated by crack growth. Using $m=3$ to do the calculation is a conservative estimate.

When we estimate the slope form the obtained fatigue data to do this statistical analysis, we will find the value of degree of improvement is higher than that value obtained by using fixed slope. 


\section{DATA SHEETS: APPLICATION OF STATISTICAL ANALYSIS OF FATIGUE}

In this data sheets, the familiar questions of statistical analysis in fatigue design are studied. In addition, the instructions of solution are shown with examples. The relative fatigue testing data is from (Marquis, 1995) and Laboratory of Fatigue and Strength in Lappeenranta University of Technology. The data form laboratory is shown in appendixXII. The data sheets are in the appendixes.

In the above chapters, the background and basic knowledge for each questions in the data sheets has been presented. The following words state the application of them:

Data sheets 6: Weibull distribution is often used in place of the normal distribution due to it is able to be generated easily without typically variates as normal distribution. That is the reason to determine whether the data set is Weibull distributed.

Data sheets 8: The validation of a selected S-N curve is based on a limited number of new fatigue tests. The tests of hypotheses and significance are applied to determine the sample size.

Data sheets 9: In the estimation of parameters of S-N curve, the suitable method needs to be selected according to the type of fatigue data. If the data sets include censored data, the appropriate statistical analysis will be more complicated.

Data sheets 10-13: Characteristic curve are developed to ensure the safety in design. Two methods are introduced to establish the design S-N curve with 
different numbers of estimated coefficients from the data.

Data sheets 15,16: When different fabrication techniques or procedures are implemented, it is necessary to ensure the improvement is obtained not only due to the sampling. Usually, we verify the means of data sets. For S-N curve, additional parameters are needed to be taken into account. 


\section{CONCLUSION AND RECOMMENDATIONS}

In this thesis project, it illustrates the basic frame of how to use statistical methods to evaluate fatigue testing data and the solution of problem in practice. I think the well planed statistical procedures in the first several sections from section 2 to section 7 and the specific instructions for special practical cases in appendixes are helpful in fatigue design.

In this work, the precondition which assumes that the fatigue life $N$ for a given stress range $S$ is log-normally distributed has been emphasized. Both of least squares method and maximum likelihood method are widely used to obtain the linear regression line of S-N curve. The maximum likelihood estimation of the sample density function has many advantages and is recommended for analyzing censored data.

The sample mean and standard deviation for the normal distribution are random variables. The sample mean is related to the student's $t$ distribution and the sample standard deviation is characterized by the chi-square distribution. There are more or less differences between the evaluation of the sample mean and the sample variance from the test data with a curve fitting method and that from theoretical distributions. It is recommended to apply a confidence level to the sample mean and deviation to get the population mean and population standard deviation. The confidence limits, prediction limits and tolerance limits are also generated to obtain characteristic values and design values of the results. They are able to be chosen according to the sample size. When we separately use the prediction limits and tolerance limits to determine the design curve base on the testing data, the comparison indicates that the second methods are more conservative.

Weibull probability graph is one of useful approaches to checking the Weibull distributional assumption. The advantage of this method is the simplicity and speed. The 
parameters of the distribution can be obtained easily, because the intercept and slope estimate of the fitted line are in fact estimates for the location and scale parameters of the distribution. At the same time, the Anderson-Darling test is illustrated in this work. This distance test is available to check the distribution assumption and is suitable for Weibull distribution.

The importance of determination of the significance of two fatigue data sets has been mentioned in this work. We need to pay more attention to the significance level of the composite hypothesis. However, we ignore that null hypothesis has always been controversial about the likelihood of rejecting. The theoretical calculation of fatigue class is proved to be an available method to approximate the degree of improvement.

As development of data sheets, there are still some questions need to be done. For example, how to determine a mean S-N curve by using Bastenaire equation? However, I wish this work can give people some conveniences to do statistical evaluation of fatigue data. 


\section{REFERENCES}

ASTM international, 2004. Standard Practice for Statistical Analysis of Linear or Linearized Stress-Life (S-N) and Strain-Life $(\in-\mathrm{N})$ Fatigue Data. E 739-91.

Al-Fawzan M, A, 2000. Methods for Estimating the Parameters of the Weibull Distribution [Online].

Available from http://interstat.statjournals.net/YEAR/2000/articles/0010001.pdf

Beyer, W.H, 1981. CRC Standard Mathematical Tables. 26 ${ }^{\text {th }}$ Edition. CRC Press, Inc. Boca Raton, Florida

Dowling N.E, 1998. Mechanical Behavior of Materials, Engineering Methods for Deformation, Fracture, and Fatigue. Second Edition. Prentice-Hall, Upper Saddle River, New Jersey:.

Dorner William W., 1999. Using Microsoft Excel for Weibull Analysis [Online]. QCI international: Qualitydigest. [Accessed 23 February 2007]

Available from: http://www.qualitydigest.com/jan99/html/weibull.html

DoD Reliability Analysis Center. Anderson-Darling: A Goodness of Fit Test for Small Samples Assumptions. Volume 10, Number 5. Available from:

http://quanterion.com/RIAC/Publications/STARTSheets/PDFFiles/A_DTest.pdf

Hobbacher, A, International institute of welding, 2005. Recommendations for Fatigue Design of Welded Joints and Components. IIW Joint Working Group XIII-XV:IIW document XIII-1965-03/XV-1127-03

International institute of welding, 2006. Guidance for the Application of the Best Practice Guide on Statistical Analysis of Fatigue Working Sheets, IIW-XIII-WG1-121r3-06

International Organization for Standardization, 2000. Fatigue Testing of Welded Components. Draft International Standard ISO/DIS 14345.

Lihavainen V., Marquis G., Estimation of Fatigue Life Improvement for Ultrasonic Impact Treated Welded Joints. Lappeenranta University of Technology.

Lihavainen V., Marquis G., Statnikov E, 1990. Fatigue Strength of A Longitudinal Attachment Improved by Ultrasonic Impact Treatment. International institute of welding. IIW Documment XIII-1990-03 
Limpert E., Stahel W., and Abbt M., 2001. Log-normal Distributions across the Sciences: Keys and Clues, BioScience, 51 (5), p. 341-352

Maddox S.J, 1993. Fatigue Assessment of Welded Structures, Pergamon, Press, Oxford, p.33-42

Mann N.R, Schafer R.E, Singpurwalla N.D, 1974. Methods for Statistical Analysis of Reliability and Life Data. John Wiley \& Sons, Canada.

Marquis, G.B, 1995. High cycle spectrum fatigue of welded components. Technical Research Center of Finland, Finland

Minitab reference manual-Release 12 for Windows. Minitab Inc. (USA). ISBN 0-925636-40-1.

Niemi E., Marquis G., Poutiainen I, 2005. Design of Plate Structures. Lappeenranta University of Technology.

NIST/SEMATECH, 2003 last updata 2006. Engineering Statistics Handbook [Online]. Available from: http://www.itl.nist.gov/div898/handbook/index.htm [Accessed 2 March 2007]

Owen D.B.,1962. Hand Book of Statistical Tables, Addison-Wesley Publishing Company, United States of America.

Wright William, P. E. 1996. Post-weld Treatment of a Welded Bridge Girder by Ultrasonic Impact Treatment. Turner-Fairbank Hightway Research Center: Federal Hightway Adminstration. McLean

Rabb, B.R., 1999. Fatigue Testing and Its Statistical Evaluation into Design Rules. Thesis(PhD), Tampere Univeristy of Technology, Pub 253

Reliasoft Corporation, 1992. Life data types [Online], Available from: http://www.weibull.com/LifeDataWeb/censored_data.htm

Schneider, C.R.A and Maddox, S.J, 2006. Best Practice Guide on Statistical Analysis of Fatigue Data. TWI Ltd.,: Granta Park, Cambridge, UK, International Institute of welding, XIII-2138-06

Soper Daniel S., Free Statistics Calculators [Online].

Available from http://www.danielsoper.com/statcalc/ [Accessed 10 February 2007]

Spiegel, Murray R., 1990. Schaum's Outline of Theory and Problems of Probability and Statistics, McGRAW-HILL Publishing Company, United States of America. 


\section{APPENDIXES}

Appendix I sheets 6: Are the data Weibull distributed using a Weibull probability graph? Appendix II sheets 8: How many results are necessary to validate a selected S-N curve? AppendixIIIsheets 9: What S-N curve can be selected form a set of non cracked samples results? Appendix IV sheets 10: How to determine a design S-N curve, slope fixed (prediction limits) Appendix V sheets 11: How to determine a design S-N curve, slop estimated (prediction limits)? AppendixVIsheets 12: How to determine a design S-N curve, slope fixed (tolerance limits)? Appendix VIIsheets 13: How to determine a design S-N curve, slop estimated (tolerance limits)? AppendixVIIIsheets 15: Are 2 experimental design S-N curves statistically equivalent? AppendixIXsheets 16: How to determine the degree of improvement produced by a post-weld treatment process?

Appendix X Table 1: One-sided tolerance limit factors $k$ for $\gamma \%=90 \% \quad f=n-1$ AppendixXITable 2 One-sided tolerance limit factors $k$ for $\gamma \%=90 \% \quad f=n-2$ AppendixXIITable 3: Fatigue testing data from Laboratory of Fatigue and Strength in LUT 


\section{Appendix I}

\begin{tabular}{|c|c|c|}
\hline IIW/IIS & Are the data Weibull distributed using a Weibull probability graph? & Sheet 6 \\
\hline
\end{tabular}

\section{General}

\section{Context}

The Weibull distribution is an important distribution especially for reliability and maintainability analysis. The graphical methods are used because of their simplicity and speed. However, it involves a great probability of error.

\section{Principle}

This test is based on the verification of the linearity of the cumulative frequency distribution using a graph with adapted scales.

The cumulative Weibull distribution function is given by: $F(x)=1-e^{-\left(\frac{x-\gamma}{\eta}\right)^{\beta}}$ where: $\beta$ is the shape parameter, $\eta$ is the scale parameter, and $\gamma$ is the location parameter.

Letting $\gamma=0$, we change the form of the cumulative Weibull distribution function equation: $\ln \left(\ln \left[\frac{1}{1-F(x)}\right]\right)=\beta \ln x-\beta \ln \eta$

Anderson-Darling statistic is used to measure the nonparametric step function (based on the plot points). The smaller Anderson-Darling values indicate that the distribution fits the data better. Then observed significance level (OSL) probability (p-value) is obtained to check the assumption. If OSL $<0.05$, the hypothesis of Weibull distribution is rejected.

\section{Condition of application}

The necessary data to determine the Weibull probability graph are the following:

The data set, $n$ values $x_{i}$ (cycles)

$F\left(x_{i}\right)$, Commonly, we choose the median rank $\frac{i-0.3}{n+0.4}$ to estimate the $F\left(x_{i}\right)$

Shape parameter $\beta$, Scale parameter $\eta$

Determine the survival probability and reliability of $i^{\text {th }}$ failure 


\begin{tabular}{|c|c|c|}
\hline $\begin{array}{c}\text { IIW/IIS } \\
\text { Com XIII-WG1 }\end{array}$ & Are the data Weibull distributed using a Weibull probability graph? & Sheet 6 \\
\hline
\end{tabular}

\section{Procedure}

\section{1. $\underline{\text { Criteria }}$}

The selected level of risk is set to $\alpha, 0.05 \%$ probability to reject a correct hypothesis

\section{Calculation}

Calculate the shape parameter $\beta$ by using least square method:

$$
\begin{aligned}
& y=A+B x \\
& Y=\ln \left(\ln \left[\frac{1}{1-F(x)}\right]\right) \quad X=\ln \left(x_{i}\right)
\end{aligned}
$$

Estimate $F\left(x_{i}\right)$ of $i$ failure- $F\left(x_{i}\right)=\frac{i-0.3}{n+0.4}$

$$
\begin{array}{ll}
B=\frac{n \sum x_{i} y_{i}-\sum x_{i} \sum y_{i}}{n \sum x_{i}^{2}-\left(\sum x_{i}\right)^{2}} & A=\frac{\sum y_{i}}{n}-B \frac{\sum x_{i}}{n} \\
B=\ldots \ldots \ldots \ldots & A=\ldots \ldots \ldots \ldots
\end{array}
$$

Shape parameter- $\beta=m$

Scale parameter- $\eta=e^{-\left(\frac{A}{\beta}\right)}$

Reliability estimate- $R(t)=e^{-\left(\frac{x}{\eta}\right)^{\beta}}$

Survival probability-1- $R_{(t)}$

A goodness of fit test: Anderson-Darling

$$
\begin{aligned}
& A D=\sum_{i=1}^{n} \frac{1-2 i}{n}\left\{\ln \left(1-\exp \left(-Z_{(i)}\right)\right)-Z_{(n+1-i)}\right\}-n \\
& Z_{(i)}=\left[x_{(i)} / \eta\right]^{\beta} \\
& A D^{*}=(1+0.2 / \sqrt{n}) A D \\
& O S L=1 /\left\{1+\exp \left[-0.1+1.24 \ln \left(A D^{*}\right)+4.48\left(A D^{*}\right)\right]\right\}
\end{aligned}
$$

\section{Conclusion}

If $\mathrm{OSL}<0.05$, the correct hypothesis of Weibull distribution is rejected. 


\begin{tabular}{|c|c|c|}
\hline $\begin{array}{c}\text { IIW/IIS } \\
\text { Com XIII-WG1 }\end{array}$ & Are the data Weibull distributed using a Weibull probability graph? & Sheet 6 \\
\hline
\end{tabular}

\section{Example}

\section{Data and formula}

\section{Samples data}

$n=29$

\begin{tabular}{|c|c|c|c|}
\hline \multicolumn{4}{|c|}{ cycles to failure } \\
\hline 521,382 & 112,910 & $1,133,000$ & 689,973 \\
\hline $1,879,752$ & $6,766,000$ & $3,675,000$ & 958,318 \\
\hline 115,816 & $6,195,271$ & $22,082,998$ & $13,475,000$ \\
\hline $10,204,041$ & $4,310,000$ & $7,154,785$ & $20,511,538$ \\
\hline $14,910,395$ & $1,015,824$ & 664,000 & 95,982 \\
\hline $10,646,018$ & $1,580,669$ & $3,816,199$ & $19,048,838$ \\
\hline $1,475,769$ & $11,541,520$ & 787,894 & 605,721 \\
\hline & & & $9,232,000$ \\
\hline
\end{tabular}

\section{Calculation}

Calculate the shape parameter $\beta$ by using least square method:

$$
\begin{aligned}
& Y=A+B X \\
& Y=\ln \left(\ln \left[\frac{1}{1-F(x)}\right]\right) \quad X=\ln \left(x_{i}\right)
\end{aligned}
$$

\begin{tabular}{|c|c|c|c|c|}
\hline Rank & $F\left(x_{i}\right)$ & $\ln \left(\ln \left(\frac{1}{1-F\left(x_{i}\right)}\right)\right)$ & $x_{i}$ & $\ln \left(x_{i}\right)$ \\
\hline 1 & 0.023809524 & -3.725645038 & 95,982 & 11.47191595 \\
\hline 2 & 0.057823129 & -2.820733108 & 112,910 & 11.63434632 \\
\hline 3 & 0.091836735 & -2.33996397 & 115,816 & 11.659758 \\
\hline 4 & 0.12585034 & -2.006163702 & 521,382 & 13.16423826 \\
\hline 5 & 0.159863946 & -1.747600408 & 605,721 & 13.31417476 \\
\hline 6 & 0.193877551 & -1.534703301 & 664,000 & 13.40603743 \\
\hline 7 & 0.227891156 & -1.352357777 & 689,973 & 13.44440775 \\
\hline 8 & 0.261904762 & -1.191772815 & 787,894 & 13.57711884 \\
\hline 9 & 0.295918367 & -1.047365219 & 958,318 & 13.77293494 \\
\hline 10 & 0.329931973 & -0.915351077 & $1,015,824$ & 13.83121066 \\
\hline
\end{tabular}




\begin{tabular}{|c|c|c|}
\hline $\begin{array}{c}\text { IIW/IIS } \\
\text { Com XIII-WG1 }\end{array}$ & Are the data Weibull distributed using a Weibull probability graph? & Sheet 6 \\
\hline
\end{tabular}

\begin{tabular}{|c|c|c|c|c|}
\hline 11 & 0.363946 & -0.79303 & $1,133,000$ & 13.94038 \\
\hline 12 & 0.397959 & -0.6784 & $1,475,769$ & 14.20469 \\
\hline 13 & 0.431973 & -0.56989 & $1,580,669$ & 14.27336 \\
\hline 14 & 0.465986 & -0.46628 & $1,879,752$ & 14.44665 \\
\hline 15 & 0.5 & -0.36651 & $3,675,000$ & 15.11706 \\
\hline 16 & 0.534014 & -0.26971 & $3,816,199$ & 15.15477 \\
\hline 17 & 0.568027 & -0.17508 & $4,310,000$ & 15.27645 \\
\hline 18 & 0.602041 & -0.08185 & $6,195,271$ & 15.6393 \\
\hline 19 & 0.636054 & 0.010694 & $6,766,000$ & 15.72742 \\
\hline 20 & 0.670068 & 0.10334 & $7,154,785$ & 15.78329 \\
\hline 21 & 0.704082 & 0.196941 & $9,232,000$ & 16.03819 \\
\hline 22 & 0.738095 & 0.292501 & $10,204,041$ & 16.13829 \\
\hline 23 & 0.772109 & 0.39129 & $10,646,018$ & 16.1807 \\
\hline 24 & 0.806122 & 0.495018 & $11,541,520$ & 16.26146 \\
\hline 25 & 0.840136 & 0.60619 & $13,475,000$ & 16.41635 \\
\hline 26 & 0.87415 & 0.728834 & $14,910,395$ & 16.51757 \\
\hline 27 & 0.908163 & 0.870349 & $19,048,838$ & 16.76252 \\
\hline 28 & 0.942177 & 1.047448 & $20,511,538$ & 16.8365 \\
\hline 29 & 0.97619 & 1.318462 & $22,082,998$ & 16.91032 \\
\hline
\end{tabular}

$$
\begin{array}{ll}
B=\frac{n \sum x_{i} y_{i}-\sum x_{i} \sum y_{i}}{n \sum x_{i}^{2}-\left(\sum x_{i}\right)^{2}} & A=\frac{\sum y_{i}}{n}-B \frac{\sum x_{i}}{n} \\
B=0.7244 & A=-11.22
\end{array}
$$

Shape parameter- $\beta=m$

0.7244

Scale parameter- $\eta=e^{-\left(\frac{A}{\beta}\right)}$

Reliability estimate- $R(t)=e^{-\left(\frac{x}{\eta}\right)^{\beta}}$

Survival probability-1- $R_{(t)}$ 


\begin{tabular}{|c|c|c|}
\hline IIW/IIS & Are the data Weibull distributed using a Weibull probability graph? & Sheet 6 \\
\hline
\end{tabular}

\begin{tabular}{|c|c|c|c|c|c|}
\hline Cycles & Reliability & $\begin{array}{c}\text { Survival } \\
\text { probability }\end{array}$ & Cycles & Reliability & $\begin{array}{c}\text { Survival } \\
\text { probability }\end{array}$ \\
\hline 521,382 & 0.830554006 & 0.169445994 & $3,675,000$ & 0.465804 & 0.534196 \\
\hline $1,879,752$ & 0.624950498 & 0.375049502 & $22,082,998$ & 0.060773 & 0.939227 \\
\hline 115,816 & 0.939476587 & 0.060523413 & $7,154,785$ & 0.289992 & 0.710008 \\
\hline $10,204,041$ & 0.20171019 & 0.79828981 & 664,000 & 0.801553 & 0.198447 \\
\hline $14,910,395$ & 0.121586325 & 0.878413675 & $3,816,199$ & 0.456054 & 0.543946 \\
\hline $10,646,018$ & 0.191884736 & 0.808115264 & 787,894 & 0.778497 & 0.221503 \\
\hline $1,475,769$ & 0.674013375 & 0.325986625 & 689,973 & 0.796571 & 0.203429 \\
\hline 112,910 & 0.940547031 & 0.059452969 & 958,318 & 0.749349 & 0.250651 \\
\hline $6,766,000$ & 0.30458684 & 0.69541316 & $13,475,000$ & 0.141119 & 0.858881 \\
\hline $6,195,271$ & 0.327823034 & 0.672176966 & $20,511,538$ & 0.070315 & 0.929685 \\
\hline $4,310,000$ & 0.424223692 & 0.575776308 & 95,982 & 0.946968 & 0.053032 \\
\hline $1,015,824$ & 0.740083541 & 0.259916459 & $19,048,838$ & 0.080762 & 0.919238 \\
\hline $1,580,669$ & 0.660587425 & 0.339412575 & 605,721 & 0.813049 & 0.186951 \\
\hline $11,541,520$ & 0.173717071 & 0.826282929 & $9,232,000$ & 0.225612 & 0.774388 \\
\hline $1,133,000$ & 0.721977173 & 0.278022827 & & & \\
\hline
\end{tabular}

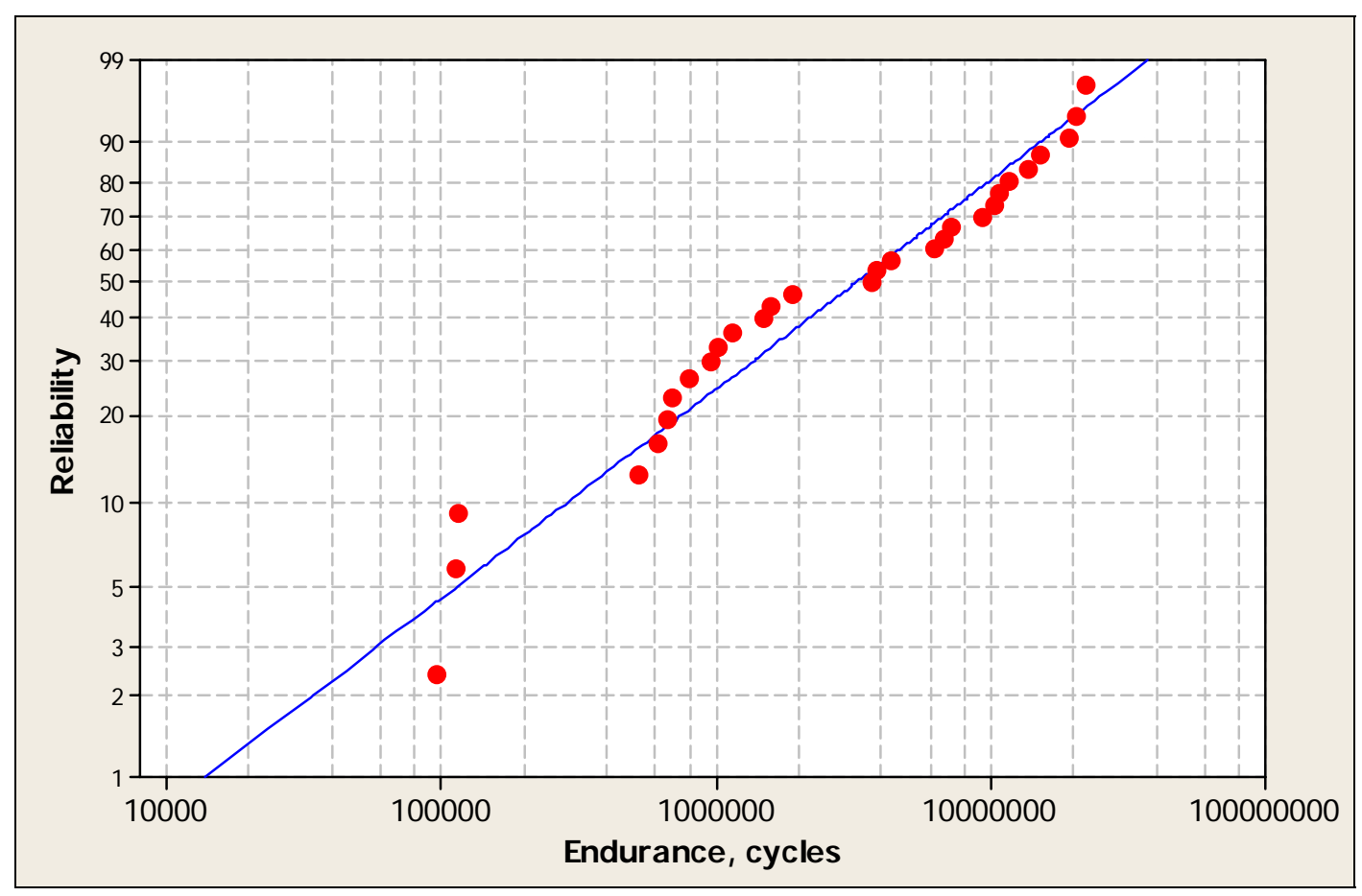

Weibull probability graph 


\begin{tabular}{|c|c|c|}
\hline $\begin{array}{c}\text { IIW/IIS } \\
\text { Com XIII-WG1 }\end{array}$ & Are the data Weibull distributed using a Weibull probability graph? & Sheet 6 \\
\hline
\end{tabular}

A goodness of fit test: Anderson-Darling

$$
\begin{aligned}
& A D=\sum_{i=1}^{n} \frac{1-2 i}{n}\left\{\ln \left(1-\exp \left(-Z_{(i)}\right)\right)-Z_{(n+1-i)}\right\}-n \\
& Z_{(i)}=\left[x_{(i)} / \eta\right]^{\beta} \\
& A D=29.4244-29=0.4244 \\
& A D^{*}=(1+0.2 / \sqrt{n}) A D=0.4402 \\
& O S L=1 /\left\{1+\exp \left[-0.1+1.24 \ln \left(A D^{*}\right)+4.48\left(A D^{*}\right)\right]\right\} \\
& O S L=0.425>\alpha=0.05
\end{aligned}
$$

\section{Conclusion}

As OSL $>0.05$ then the data is Weibull distributed. 


\section{Appendix II}

\begin{tabular}{|c|c|c|}
\hline $\begin{array}{c}\text { IIW/IIS } \\
\text { Com XIII-WG1 }\end{array}$ & How many results are necessary to validate a selected S-N curve? & Sheet 8 \\
\hline
\end{tabular}

\section{General}

\section{Context}

In fatigue testing, we would like to take enough observations to obtain reasonably precise estimates of the interested parameters, but we also want to do it within a practical resource budget.

\section{Principle}

We estimate the mean of population to determine the minimum sample size. When sample data is collected and the sample mean is calculated. The margin of error $\delta$ is the maximum difference between the observed sample mean and the population mean. $n=\left(z_{\alpha}+z_{\beta}\right)^{2}\left[\frac{\sigma}{\delta}\right]^{2}$ One-sided test [1] where: the positive $z$ value is at the vertical boundary in the right tail of the standard normal distribution. To control the risk of accepting a false hypothesis, $\beta$ is the probability of accepting the null hypothesis. $\sigma$ is the population standard deviation; $n$ is the sample size.

Validating tests performed to S-N curve:

$\overline{\log A_{\text {test }}} \geq \overline{\log A}+\frac{1.645 \sigma}{\sqrt{n}}$

where: $\overline{\log A_{\text {test }}}$ is the mean logarithm of interception from the tests. $\overline{\log A}$ is the logarithm of the interception from the mean for the selected S-N curve and the value 1.645 is obtained from standard normal probability tables for a probability of 0.95 .

\section{Condition of application}

- The slope of the mean S-N curve for the new test results is the same as $m$ of the selected curve.

- The standard deviation $\sigma_{\text {test }}$ of $\log \mathrm{N}$ about the mean $\mathrm{S}-\mathrm{N}$ curve for the new tests is the same as that for the main database. 


\begin{tabular}{|c|c|c|}
\hline $\begin{array}{c}\text { IIW/IIS } \\
\text { Com XIII-WG1 }\end{array}$ & How many results are necessary to validate a selected S-N curve? & Sheet 8 \\
\hline
\end{tabular}

\section{Procedure}

\section{Criteria}

For other significance levels, different values are obtained from the table:

\begin{tabular}{|c|c|}
\hline $10 \%$ & Level of significance: 1.285 \\
\hline $5 \%$ & Level of significance: 1.645 \\
\hline $2.5 \%$ & level of significance: 1.96 \\
\hline $1 \%$ & Level of significance: 2.33 \\
\hline
\end{tabular}

Note that the corresponding level of significance of $5 \%$ is commonly considered to give a sufficiently low probability of concluding that the populations are different in the case where they are actually that same.

\section{Data and formula}

Validation the use of Class D at the 5\% level of significance:

Mean S-N curve for Class D- $S^{3} N=3.99 \times 10^{12}$

Standard deviation of $\log N-\sigma=0.2097$

Slop of design curve- $m$

Sum of values- $\sum \log N$

Mean value- $\overline{\log N}=\frac{\sum \log N}{n}$

Sum of values- $\sum \log S$

Mean value- $\overline{\log S}=\frac{\sum \log S}{n}$

According to $\overline{\log A}=\overline{\log N}+m \overline{\log S}$, mean value- $\overline{\log A}$

Transform the Eq.[2] into the form as Eq.[1]:

$$
n \geq\left(z_{\alpha / 2}+z_{\beta}\right)^{2}\left(\frac{\sigma}{\overline{\log A_{\text {test }}}-\overline{\log A}}\right)^{2}
$$




\begin{tabular}{|c|c|c|}
\hline $\begin{array}{c}\text { IIW/IIS } \\
\text { Com XIII-WG1 }\end{array}$ & How many results are necessary to validate a selected S-N curve? & Sheet 8 \\
\hline
\end{tabular}

\section{Example}

\section{Data and formula}

\section{Samples data}

$n=29$

\begin{tabular}{|c|c|c|c|}
\hline Stress range & cycles to failure & Stress range & cycles to failure \\
\hline 147 & 521,382 & 74 & $3,675,000$ \\
\hline 96 & $1,879,752$ & 53 & $22,082,998$ \\
\hline 250 & 115,816 & 53 & $7,154,785$ \\
\hline 61 & $10,204,041$ & 136 & 664,000 \\
\hline 57 & $14,910,395$ & 75 & $3,816,199$ \\
\hline 57 & $10,646,018$ & 136 & 787,894 \\
\hline 96 & $1,475,769$ & 136 & 689,973 \\
\hline 250 & 112,910 & 147 & 958,318 \\
\hline 74 & $6,766,000$ & 53 & $13,475,000$ \\
\hline 74 & $6,195,271$ & 53 & $20,511,538$ \\
\hline 74 & $4,310,000$ & 265 & 95,982 \\
\hline 136 & $1,015,824$ & 54 & $19,048,838$ \\
\hline 136 & $1,580,669$ & 176 & 605,721 \\
\hline 53 & $11,541,520$ & 74 & $9,232,000$ \\
\hline 136 & $1,133,000$ & & \\
\hline
\end{tabular}

\section{Calculation}

Validation the use of Class D S-N curve at the 5\% level of significance:

Mean S-N curve for Class D- $S^{3} N=3.99 \times 10^{12}$

Standard deviation of $\log N-\sigma=0.2097$

Slop of design curve- $m$

Mean value- $\overline{\log A}$

Sum of values- $\sum \log N_{\text {test }}$

Mean value- $\overline{\log N_{\text {test }}}=\frac{\sum \log N_{\text {test }}}{n}$ 


\begin{tabular}{|c|c|c|}
\hline IIW/IIS & How many results are necessary to validate a selected S-N curve? & Sheet 8 \\
\hline
\end{tabular}

Sum of values- $\sqrt{\log S_{\text {test }}}$

Mean value- $\overline{\log S_{\text {test }}}=\frac{\sum \log S_{\text {test }}}{n}$

According to $\overline{\log A}=\overline{\log N}+m \overline{\log S}$, mean value- $\overline{\log A_{\text {test }}}$

Transform the Eq.[2] into the form as Eq.[1]:

$n \geq\left(z_{\alpha}+z_{\beta}\right)^{2}\left(\frac{\sigma}{\overline{\log A_{\text {test }}}-\overline{\log A}}\right)^{2} \quad \alpha=5 \% \quad \beta=10 \%$ $n \geq(1.645+1.285)^{2}\left(\frac{0.2097}{12.335-12.601}\right)^{2}$

$n \geq 5.335$

\section{Conclusion}

According to above calculations, the minimum number of sample size to validate the selected class D S-N curve should not be less than 6 . 


\section{AppendixIII}

\begin{tabular}{|c|c|c|}
\hline $\begin{array}{c}\text { IIW/IIS } \\
\text { Com XIII-WG1 }\end{array}$ & What S-N cure can be selected from a set of non cracked results? & Sheet 9 \\
\hline
\end{tabular}

\section{General}

\section{Contest}

Commonly, we assumed that each specimen in the test yielded an exact failure. Actually, the results or parts of specimens which are obtained from specimens have not failed. Those unfailed or non cracked specimen are often termed 'run-outs'.

It is known that under constant amplitude loading there is a fatigue endurance defined as the stress range below which failure will not occur. For design purpose, we make the S-N curve extend down to the constant amplitude fatigue limit and turn to a horizontal line. However, in practice fatigue test results usually follow an S-N curve that gradually changes slope in the region of the constant amplitude fatigue limit.

\section{Principle}

Various statistical methods can be used to analyze the endurance test results, determined statistical parameters. Based on the working group 1 of Commission XII of the International Institute of Welding, those statistical methods can be sorted as the following table according to the each main characteristic.

\begin{tabular}{|c|c|c|c|}
\hline $\begin{array}{l}\text { Method } \\
\text { of } \\
\text { Analysis }\end{array}$ & S-N curve equation & Method and Assumptions & Parameters \\
\hline (A) & $\log N=\log C-m \log S$ & $\begin{array}{l}\text { Mean line bisecting the two regression } \\
\text { lines }\end{array}$ & $C, m,{ }^{s} \log S$ \\
\hline (B) & $\log N=\log C-m \log S$ & $\begin{array}{c}\text { Linear regression of } \log \mathrm{N} \text { on } \log \mathrm{S} \\
\text { ignoring run-out's }\end{array}$ & $C, m,{ }^{s} \log S$ \\
\hline (C) & $\log N=\log C-m \log S$ & Maximum likelihood (includes run-out's) & $C, m,{ }^{\mathrm{s}} \log S$ \\
\hline (D) & $\log N=\log C-m \log S$ & $\begin{array}{l}\text { Linear regression of } \log \mathrm{N} \text { on } \log \mathrm{S} \\
\text { run-out's being included }\end{array}$ & $C, m,{ }^{s} \log S$ \\
\hline$(\mathrm{E})$ & $N=\frac{A}{S-E}\left[-\frac{(S-E) C}{B}\right]$ & $\begin{array}{c}\text { Multiple non-linear regression } \\
\text { including censored data (run-out's) } \\
\text { Stress response curves: sigmoid normal }\end{array}$ & $\begin{array}{l}A, B, E \\
{ }^{\mathrm{s}} \mathrm{S}, F\end{array}$ \\
\hline
\end{tabular}




\begin{tabular}{|c|c|c|}
\hline $\begin{array}{c}\text { IIW/IIS } \\
\text { Com XIII-WG1 }\end{array}$ & What S-N cure can be selected from a set of non cracked results? & Sheet 9 \\
\hline
\end{tabular}

\section{Method A}

Definition of the S-N curve by drawing bisecting line of the straight lines plotted from the regression of $\log N$ against $\log S$ and of $\log S$ against $\log N$ respectively.

\section{Method B}

Conventional plotting of $\log N-\log S$

\section{Method C}

By using the maximum likelihood method, straight lines can be plotted in a $\log S-\log N$ coordinate system. It is an appropriate tool for solving the general problem of estimating the 'best fit' line through censored test data. In general case, numerical iteration is required to derive maximum likelihood estimates. In special case of exact data, the maximum likelihood method leads to the least squares function on which linear regression is generally based.

This method amounts to curve fitting in compliance with the formula:

$$
N \times S^{n}=K
$$

\section{Method D and E}

BASTENAIRE's method provides for fitting particularly any model of S-N curve by supplementing a basal computer program with sub-programs. Two models may be considered:

$$
\begin{array}{ll}
N \times S^{m}=C & \text { Method D } \\
N(S-E)=A \exp [-(S-E) / B]^{c} & \text { Method E }
\end{array}
$$

where: $\mathrm{S}$ is the nominal stress and $N$ the corresponding number of cycles to failure, A, B, C and E are statistically assessed parameters. 


\begin{tabular}{|c|c|c|}
\hline $\begin{array}{c}\text { IIW/IIS } \\
\text { Com XIII-WG1 }\end{array}$ & What S-N cure can be selected from a set of non cracked results? & Sheet 9 \\
\hline
\end{tabular}

\section{Condition of application}

- In statistical analysis, we are able to decide whether the results from unfailed specimens can be used according to the circumstance.

- Test results from either failed or unfailed specimens that lie in this transition region approaching the constant amplitude fatigue limit should not be combined with those obtained at higher stresses in the estimation of the best-fit linear S-N curve.

- Depending on this guide, notched or welded specimens that give $N<2 \times 10^{6}$ cycles could be fall into this category, for smooth specimen $N<10^{6}$.

- In addition, fatigue test results from unfailed specimens can be used to estimation of the best-fit S-N curve in two other situations:

a) The test is stopped deliberately.

b) The test specimen contains more than one site for potential fatigue failure and fails from just one of them.

\section{Conclusion}

The methods A and B do not take unbroken specimens into account. While other three methods consider the run-outs in the analysis and which have a bearing on the final results. But when the fatigue data include either run-outs or suspended test, the appropriate statistical analyses become more complicated. Fortunately, many statistical software packages can perform the required calculations.

At the same time, methods based on the formula $N \times S^{m}=C$ yield strongly variable results if parameters $C$ and $m$ are taken into consideration; while the deviation between the $S$ values is smaller for a given fatigue life. 


\begin{tabular}{|c|c|c|}
\hline $\begin{array}{c}\text { IIW/IIS } \\
\text { Com XIII-WG1 }\end{array}$ & What S-N cure can be selected from a set of non cracked results? & Sheet 9 \\
\hline
\end{tabular}

When we test a number of specimens which contain the same number of nominally identical welds and any of which might fail first. If each specimen is tested until it fails at exactly one of the potential locations, then the S-N curve for a single weld can be established by using least squares estimation, together with the tabulated extreme value statistics for normal distribution. 


Appendix IV
\begin{tabular}{|c|c|c|}
\hline $\begin{array}{c}\text { IIW/IIS } \\
\text { Com XIII-WG1 }\end{array}$ & How to determine a design S-N curve, slope fixed (prediction limits)? & Sheet 10 \\
\hline
\end{tabular}

\section{General}

\section{1. $\underline{\text { Contest }}$}

For design purpose, it is necessary to establish limits between which a given proportion (typically 95\%) of the data lies. Prediction limits are used to avoid confusion with the confidence limits on the coefficients of the regression line. The interval between the upper and lower prediction limits is called a prediction interval.

\section{Principle}

Prediction limits at stress range $S$ can be expressed explicitly, in the form:

$$
\log N_{p \%}^{ \pm}=(\log A-m \log S) \pm t \hat{\sigma} \sqrt{1+\frac{1}{n}+\frac{(\log S-\overline{\log S})^{2}}{\sum_{i=1}^{n}\left(\log S_{i}-\overline{\log S}\right)^{2}}}
$$

where: $\log A$ and $m$ are the coefficients of the regression line through the $n$ data points

$\overline{\log S}$ is the mean of the $n$ values of $\log S_{i}$

$t \quad$ is the appropriate percentage point of Student's $t$ distribution

$\hat{\sigma}$ is the best estimate of the variance of the data about the regression line,

$f \quad$ is degrees of freedom

\section{Condition of application}

- It is often assumed that design curves will only be applied to values of $\log S$ that are not far from the mean value. In this case the third term under the square root in the equation can be ignored.

- When slope $m$ is chosen to take a fixed value. The number of degrees of freedom should be $n-1$. Whenever the sample sizes is less than ten it is recommended. The expression under the square root is exactly equal to one.

- When the sample size is larger than 20, the second term under the square root is able to be ignored. 


\begin{tabular}{|c|c|c|}
\hline $\begin{array}{c}\text { IIW/IIS } \\
\text { Com XIII-WG1 }\end{array}$ & How to determine a design S-N curve, slope fixed (prediction limits)? & Sheet 10 \\
\hline
\end{tabular}

\section{Procedure}

\section{1. $\underline{\text { Criteria }}$}

The design curves will only be applied to values of $\log S$ that are not far removed from the mean value $\overline{\log S}$.

In general welding case, we use a gradient of $m=3$.

\section{Data and formula}

Using the number of stress range, load cycles, and $\log A=\overline{\log N}+m \overline{\log S}$ to obtain:

Mean of Intercept of regression line- $\log A$

Slop of design curve- $m$

Sample size- $n$

Sum of values- $\sum \log S_{i}$

Mean values- $\overline{\log S}=\frac{\sum \log S_{i}}{n}$

Sum of values- $\sum \log N_{i}$

Mean values- $\overline{\log N}=\frac{\sum N_{i}}{n}$

Estimated mean of Intercept of regression line- $\log \hat{A}$

Estimated value- $\log \hat{N}_{i}=\log \hat{A}-3 \times \log S_{i}$

Estimated the variance $-\hat{\sigma}^{2}=\frac{\sum_{i=1}^{n}\left(\log N_{i}-\log \hat{N}_{i}\right)^{2}}{f}$

Degree of freedom- $f=n-1$ 


\begin{tabular}{|c|c|c|}
\hline $\begin{array}{c}\text { IIW/IIS } \\
\text { Com XIII-WG1 }\end{array}$ & How to determine a design S-N curve, slope fixed (prediction limits)? & Sheet 10 \\
\hline
\end{tabular}

$$
\hat{\sigma}=\sqrt{\frac{\sum\left(\log N_{i}-\log \hat{N}_{i}\right)^{2}}{f}}
$$

Appropriate percentage point of two sided Student's $t$ distribution- $t$

Design S-N curve: $\log N_{p \%}^{ \pm}=(\log A-m \log S) \pm t \hat{\sigma}$ 


\begin{tabular}{|c|c|c|}
\hline $\begin{array}{c}\text { IIW/IIS } \\
\text { Com XIII-WG1 }\end{array}$ & How to determine a design S-N curve, slope fixed (prediction limits)? & Sheet 10 \\
\hline
\end{tabular}

\section{Example}

\section{Data and formula}

\section{Samples data}

$n=9$
\begin{tabular}{|c|c|}
\hline Stress range & cycles to failure \\
\hline 147 & 521,382 \\
\hline 96 & $1,879,752$ \\
\hline 250 & 115,816 \\
\hline 61 & $10,204,041$ \\
\hline 57 & $14,910,395$ \\
\hline 57 & $10,646,018$ \\
\hline 96 & $1,475,769$ \\
\hline 250 & 112,910 \\
\hline 74 & $6,766,000$ \\
\hline
\end{tabular}

\section{Calculation}

Proportion 95\%

Using the number of stress range, load cycles, and $\log A=\overline{\log N}+m \overline{\log S}$ to obtain

Mean of Intercept of regression line- $\log A$

Slop of design curve- $m$

Sample size- $n$

Sum of values- $\sum \log S_{i}$

Mean values- $\overline{\log S}=\frac{\sum \log S_{i}}{n}$

Sum of values- $\sum \log N_{i}$

Mean values- $\overline{\log N}=\frac{\sum N_{i}}{n}$ 


\begin{tabular}{|c|c|c|}
\hline $\begin{array}{c}\text { IIW/IIS } \\
\text { Com XIII-WG1 }\end{array}$ & How to determine a design S-N curve, slope fixed (prediction limits)? & Sheet 10 \\
\hline
\end{tabular}

Estimated value- $\log \hat{N}_{i}=\log \hat{A}-3 \times \log S_{i}$

Estimated the variance- $\hat{\sigma}^{2}=\frac{\sum_{i=1}^{n}\left(\log N_{i}-\log \hat{N}_{i}\right)^{2}}{f}$

Degree of freedom- $f=n-1$

$\hat{\sigma}=\sqrt{\frac{\sum\left(\log N_{i}-\log \hat{N}_{i}\right)^{2}}{f}}$

Appropriate percentage point of two sided Student's $t$ distribution- $t$

Design S-N curve: $\log N_{p \%}^{ \pm}=(\log A-m \log S) \pm t \hat{\sigma} \sqrt{1+\frac{1}{n}}$

$$
\begin{aligned}
& \log N_{95 \%}^{ \pm}=(12.2889-3 \log S) \pm 2.306 \times 0.108 \times \sqrt{1+\frac{1}{9}} \\
& \log N_{95 \%}^{ \pm}=(12.2889-3 \log S) \pm 0.2625
\end{aligned}
$$

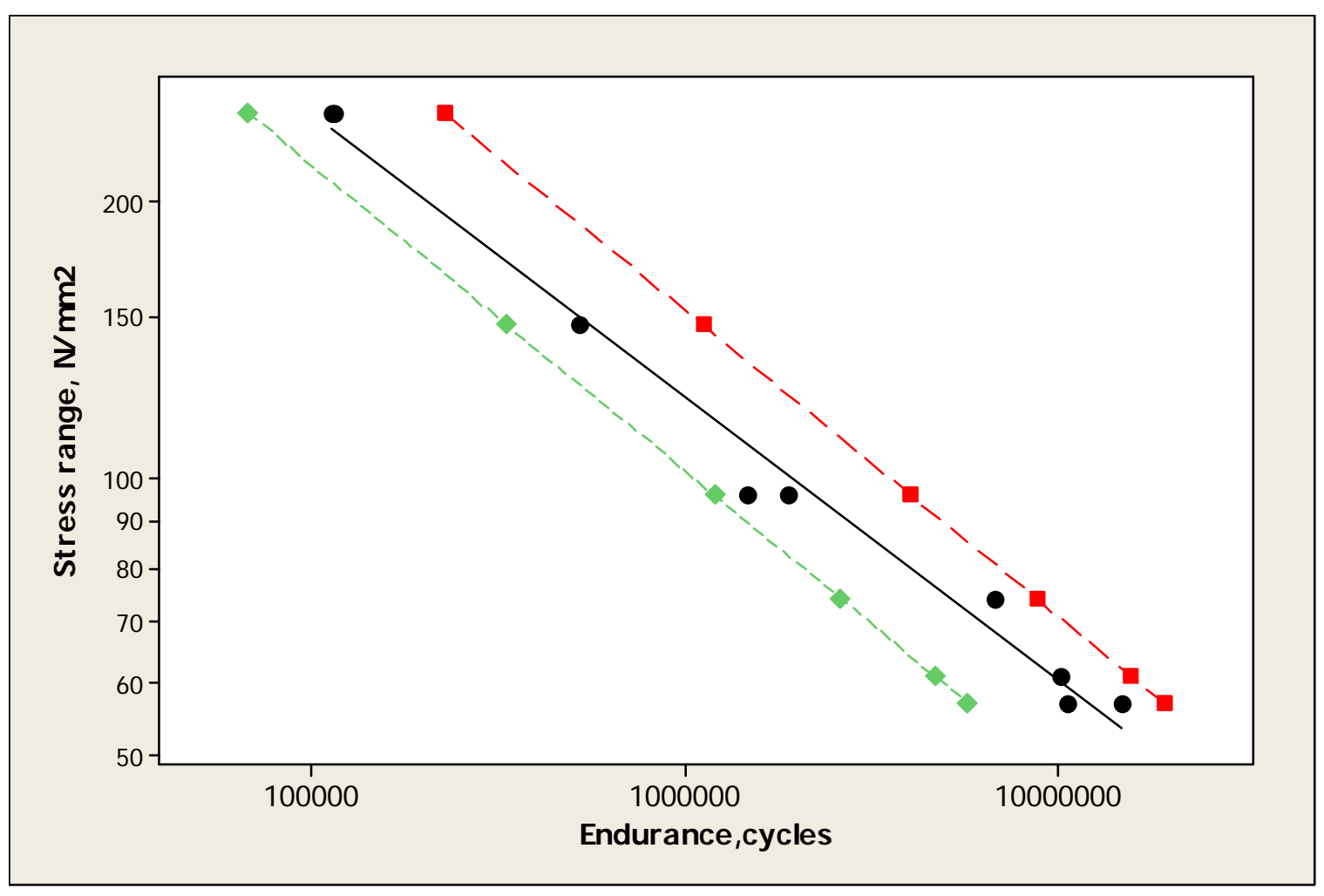

Design curve based on prediction limits (slope fixed) 


\section{Appendix V}

\begin{tabular}{|c|c|c|}
\hline $\begin{array}{c}\text { IIW/IIS } \\
\text { Com XIII-WG1 }\end{array}$ & $\begin{array}{c}\text { How to determine a design S-N curve, slop estimated } \\
\text { (prediction limits)? }\end{array}$ & Sheet 11 \\
\hline
\end{tabular}

\section{General}

\section{1. $\underline{\text { Contest }}$}

For design purpose, it is necessary to establish limits between which a given proportion (typically 95\%) of the data lies. The instruction for determination of a design S-N curve based on prediction limits has been introduced in Data Sheet10. But it focuses on the slop fixed case. When both of the slop and intercept are needed to be estimated, some changes are needed to be emphasized.

\section{Principle}

Prediction limits at stress range $S$ can be expressed explicitly, in the form:

$$
\log N_{p \%}^{ \pm}=(\log A-m \log S) \pm t \hat{\sigma} \sqrt{1+\frac{1}{n}+\frac{(\log S-\overline{\log S})^{2}}{\sum_{i=1}^{n}\left(\log S_{i}-\overline{\log S}\right)^{2}}}
$$

where: $\log A$ and $m$ are the coefficients of the regression line through the $n$ data points

$\overline{\log S}$ is the mean of the $n$ values of $\log S_{i}$

$t$ is the appropriate percentage point of Student's $t$ distribution

$\hat{\sigma}$ is the best estimate of the variance of the data about the regression line,

$f$ is degree of freedom, $f=n-2$ in case where the two coefficients of the regression line have been estimated from the data

Additionally, the Maximum likelihood estimation is used to estimate the slope and intercept coefficients.

\section{Condition of application}

- In general, it is often assumed that design curves will only be applied to values of $\log S$ that are not far from the mean value $\overline{\log S}$.In this case the third term under the square root of equation can be ignored

- When the sample size larger than 20. Then the second term under the square root is able to be ignored. 


\begin{tabular}{|c|c|c|}
\hline $\begin{array}{c}\text { IIW/IIS } \\
\text { Com XIII-WG1 }\end{array}$ & $\begin{array}{c}\text { How to determine a design S-N curve, slop estimated } \\
\text { (prediction limits)? }\end{array}$ & Sheet 11 \\
\hline
\end{tabular}

\section{Procedure}

\section{1. $\underline{\text { Criteria }}$}

The design curves will only be applied to values of $\log S$ that are not far removed from the mean value $\overline{\log S}$

\section{Data and formula}

The correct values for the constants $A$ and $m$ are obtained from the following two equations by using the maximum likelihood method:

$$
\begin{aligned}
& \log A=\overline{\log N}+m \overline{\log S} \\
& m=\frac{\sum_{i=1}^{n}\left(\log S_{i}-\overline{\log S}\right)\left(\log N_{i}-\overline{\log N}\right)}{\sum_{i=1}^{n}\left(\log S_{i}-\overline{\log S}\right)^{2}}
\end{aligned}
$$

Sample size- $n$

Degree of freedom- $f=n-2$

Sum of values- $\sum \log S_{i}$

Mean values- $\overline{\log S}=\frac{\sum \log S_{i}}{n}$

Sum of values- $\sum \log N_{i}$

Mean values- $\overline{\log N}=\frac{\sum N_{i}}{n}$

$$
\sum_{i=1}^{n}\left(\log S_{i}-\overline{\log S}\right)\left(\log N_{i}-\overline{\log N}\right)
$$

$\sum_{i=1}^{n}\left(\log S_{i}-\overline{\log S}\right)^{2}$ 


\begin{tabular}{|c|c|c|}
\hline $\begin{array}{c}\text { IIW/IIS } \\
\text { Com XIII-WG1 }\end{array}$ & $\begin{array}{c}\text { How to determine a design S-N curve, slop estimated } \\
\text { (prediction limits)? }\end{array}$ & Sheet 11 \\
\hline
\end{tabular}

Estimated slope- $\hat{m}$

Estimated mean of Intercept of regression line- $\log \hat{A}$

Estimated value- $\log \hat{N}_{i}=\log \hat{A}-\hat{m} \times \log S_{i}$

Estimated the variance- $\hat{\sigma}^{2}=\frac{\sum_{i=1}^{n}\left(\log N_{i}-\log \hat{N}_{i}\right)^{2}}{f}$

$$
\hat{\sigma}=\sqrt{\frac{\sum\left(\log N_{i}-\log \hat{N}_{i}\right)^{2}}{f}}
$$

Appropriate percentage point of two sided Student'st distribution- $t$

Design S-N curve: $\log N_{p \%}^{ \pm}=(\log A-m \log S) \pm t \hat{\sigma} \sqrt{1+\frac{1}{n}}$ 


\begin{tabular}{|c|c|c|}
\hline $\begin{array}{c}\text { IIW/IIS } \\
\text { Com XIII-WG1 }\end{array}$ & $\begin{array}{c}\text { How to determine a design S-N curve, slop estimated } \\
\text { (prediction limits)? }\end{array}$ & Sheet 11 \\
\hline
\end{tabular}

\section{Example}

\section{Data and formula}

\section{Samples data}

$n=29$

\begin{tabular}{|c|c|c|c|}
\hline Stress range & cycles to failure & Stress range & cycles to failure \\
\hline 147 & 521,382 & 74 & $3,675,000$ \\
\hline 96 & $1,879,752$ & 53 & $22,082,998$ \\
\hline 250 & 115,816 & 53 & $7,154,785$ \\
\hline 61 & $10,204,041$ & 136 & 664,000 \\
\hline 57 & $14,910,395$ & 75 & $3,816,199$ \\
\hline 57 & $10,646,018$ & 136 & 787,894 \\
\hline 96 & $1,475,769$ & 136 & 689,973 \\
\hline 250 & 112,910 & 147 & 958,318 \\
\hline 74 & $6,766,000$ & 53 & $13,475,000$ \\
\hline 74 & $6,195,271$ & 53 & $20,511,538$ \\
\hline 74 & $4,310,000$ & 265 & 95,982 \\
\hline 136 & $1,015,824$ & 54 & $19,048,838$ \\
\hline 136 & $1,580,669$ & 176 & 605,721 \\
\hline 53 & $11,541,520$ & 74 & $9,232,000$ \\
\hline 136 & $1,133,000$ & & \\
\hline & & & \\
\hline
\end{tabular}

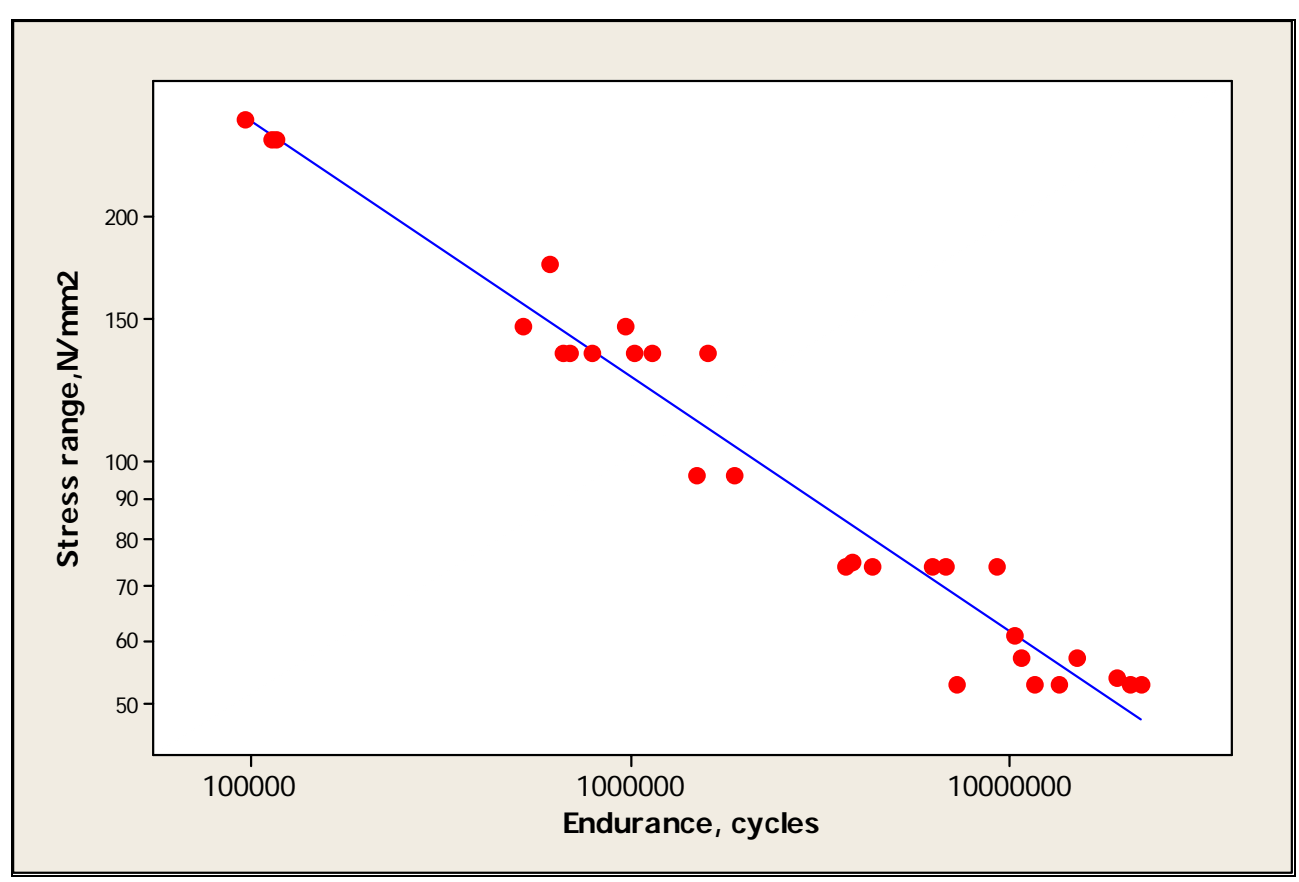

Linear regression S-N curve 


\begin{tabular}{|c|c|c|}
\hline $\begin{array}{c}\text { IIW/IIS } \\
\text { Com XIII-WG1 }\end{array}$ & $\begin{array}{c}\text { How to determine a design S-N curve, slop estimated } \\
\text { (prediction limits)? }\end{array}$ & Sheet 11 \\
\hline
\end{tabular}

\section{Calculation}

Proportion 95\%

The correct values for the constants $A$ and $k$ are obtained from the following two equations by using the maximum likelihood method:

$$
\begin{aligned}
& \log \hat{A}=\overline{\log N}+m \overline{\log S} \\
& \hat{m}=-\frac{\sum_{i=1}^{n}\left(\log S_{i}-\overline{\log S}\right)\left(\log N_{i}-\overline{\log N}\right)}{\sum_{i=1}^{n}\left(\log S_{i}-\overline{\log S}\right)^{2}}
\end{aligned}
$$

Sample size- $n$

Degree of freedom- $f=n-2$

Sum of values- $\sum \log S_{i}$

Mean values- $\overline{\log S}=\frac{\sum \log S_{i}}{n}$

Sum of values- $\sum \log N_{i}$

Mean values- $\overline{\log N}=\frac{\sum N_{i}}{n}$

$\sum_{i=1}^{n}\left(\log S_{i}-\overline{\log S}\right)\left(\log N_{i}-\overline{\log N}\right)$

$\sum_{i=1}^{n}\left(\log S_{i}-\overline{\log S}\right)^{2}$

Estimated slope- $\hat{m}$

Estimated mean of Intercept of regression line- $\log \hat{A}$

Estimated value- $\log \hat{N}_{i}=\log \hat{A}-\hat{m} \times \log S_{i}$ 


\begin{tabular}{|c|c|c|}
\hline $\begin{array}{c}\text { IIW/IIS } \\
\text { Com XIII-WG1 }\end{array}$ & $\begin{array}{c}\text { How to determine a design S-N curve, slop estimated } \\
\text { (prediction limits)? }\end{array}$ & Sheet 11 \\
\hline
\end{tabular}

Estimated the variance- $\hat{\sigma}^{2}=\frac{\sum_{i=1}^{n}\left(\log N_{i}-\log \hat{N}_{i}\right)^{2}}{f}$

$\hat{\sigma}=\sqrt{\frac{\sum\left(\log N_{i}-\log \hat{N}_{i}\right)^{2}}{f}}$

Appropriate percentage point of two sided Student's $t$ distribution- $t$

Design S-N curve: $\log N_{p \%}^{ \pm}=(\log A-m \log S) \pm t \hat{\sigma} \sqrt{1+\frac{1}{n}}$

$$
\begin{aligned}
& n=29>20 \\
& \log N_{p \%}^{ \pm}=(\log A-m \log S) \pm t \hat{\sigma} \\
& \log N_{95 \%}^{ \pm}=(12.4062-3.036 \log S) \pm 2.052 \times 0.1465 \\
& \log N_{95 \%}^{ \pm}=(12.4062-3.036 \log S) \pm 0.301
\end{aligned}
$$

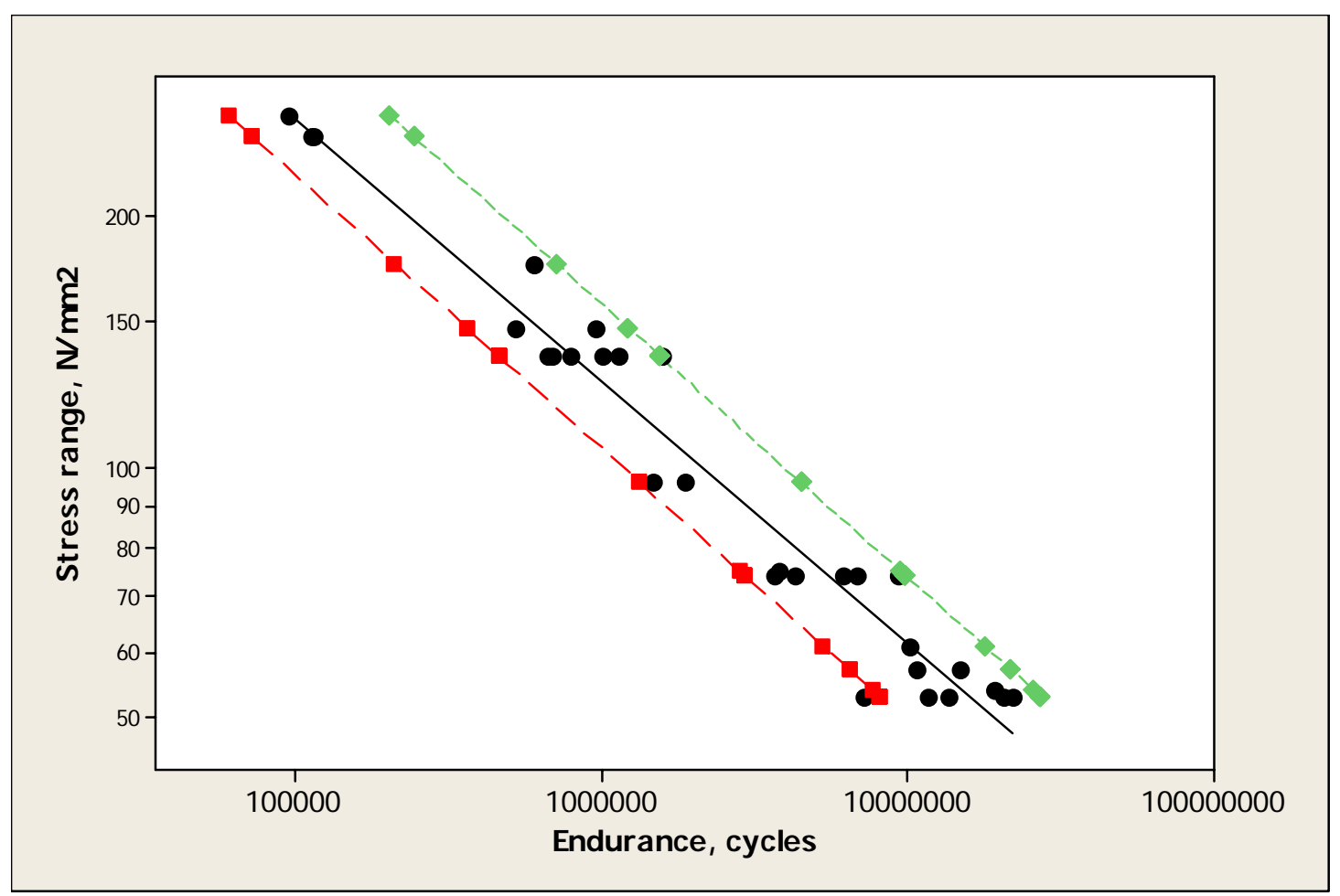

Design curve based on prediction limits (slope estimated) 


\section{Appendix VI}

\begin{tabular}{|c|l|l|}
$\begin{array}{c}\text { IIW/IIS } \\
\text { Com XIII-WG1 }\end{array}$ & How to determine a design S-N curve, slope fixed (tolerance limits)? & Sheet 12 \\
\hline
\end{tabular}

\section{General}

\section{Contest}

The use of tolerance limits rather than prediction limits would yield a more conservative design curve and they have the advantage that they explicitly allow for uncertainty in estimates of population statistics from a small sample.

\section{Principle}

This statement is made on the basis of a sample of $n$ independent observations. A tolerance limit can be regarded as a confidence limit on a prediction limit. $\log N_{p \%}^{-}=\hat{\mu}-k s$

where: $\hat{\mu}$ is an estimate of the mean $\log$ of the endurance at stress $S$

$s$ is an estimate of the standard deviation of the log of the endurance at stress $S$, based on $f$ degree of freedom

$k$ is a one-sided tolerance limit factor

Tolerance limits stated that at least a proportion of normal population is greater than $\hat{\mu}-k s$ with confidence $\gamma$.

In this slope fixed case, the one sided tolerance limit factor $k$ has been tabulated in the appendix $\mathrm{x}$ table 1 .

\section{Condition of application}

- It is assumed that design curves will only be applied to values of $\log S$ that are not far removed from the mean value $\overline{\log S}$.

- When slope $m$ is chosen to take a fixed value. The number of degrees of freedom should be increased by one. Whenever the sample sizes is less than ten it is recommended. 


\begin{tabular}{|c|l|l|}
\hline $\begin{array}{c}\text { IIW/IIS } \\
\text { Com XIII-WG1 }\end{array}$ & How to determine a design S-N curve, slope fixed (tolerance limits)? & Sheet 12 \\
\hline
\end{tabular}

\section{Procedure}

\section{Criteria}

A normal distribution for $\gamma \%=90 \%$, Proportion $95 \%$

The design curves will only be applied to values of $\log S$ that are not far removed from the mean value $\overline{\log S}$

\section{Data and formula}

Using the number of stress range, load cycles, and $\log A=\overline{\log N}+m \overline{\log S}$ to obtain mean of Intercept of regression line- $\log A$

Slop of design curve- $m$

Sample size- $n$

Sum of values- $\sum \log S_{i}$

Mean values- $\overline{\log S}=\frac{\sum \log S_{i}}{n}$

Sum of values- $\sum \log N_{i}$

Mean values- $\overline{\log N}=\frac{\sum N_{i}}{n}$

Estimated mean of Intercept of regression line- $\log \hat{A}$

Estimated value- $\log \hat{N}_{i}=\log \hat{A}-3 \times \log S_{i}$

Estimated the variance- $\hat{\sigma}^{2}=\frac{\sum_{i=1}^{n}\left(\log N_{i}-\log \hat{N}_{i}\right)^{2}}{f}$

Degree of freedom- $f=n-1$ 


\begin{tabular}{|c|l|l|}
\hline $\begin{array}{c}\text { IIW/IIS } \\
\text { Com XIII-WG1 }\end{array}$ & How to determine a design S-N curve, slope fixed (tolerance limits)? & Sheet 12 \\
\hline
\end{tabular}

$$
\hat{\sigma}=\sqrt{\frac{\sum_{i=1}^{n}\left(\log N_{i}-\log \hat{N}_{i}\right)^{2}}{f}}
$$

One-sided tolerance limit factor- $k$

Design S-N curve: $\log N_{p \%}^{-}=\hat{\mu}-k s$ 


\begin{tabular}{|c|c|c|}
\hline $\begin{array}{c}\text { IIW/IIS } \\
\text { Com XIII-WG1 }\end{array}$ & How to determine a design S-N curve, slope fixed (tolerance limits)? & Sheet 12 \\
\hline
\end{tabular}

\section{Example}

\section{Data and formula}

\section{Samples data}

$n=9$

\begin{tabular}{|c|c|}
\hline Stress range & cycles to failure \\
\hline 147 & 521,382 \\
\hline 96 & $1,879,752$ \\
\hline 250 & 115,816 \\
\hline 61 & $10,204,041$ \\
\hline 57 & $14,910,395$ \\
\hline 57 & $10,646,018$ \\
\hline 96 & $1,475,769$ \\
\hline 250 & 112,910 \\
\hline 74 & $6,766,000$ \\
\hline
\end{tabular}

\section{Calculation}

Proportion 95\%

Using the number of stress range, load cycles, and $\log A=\overline{\log N}+m \overline{\log S}$ to obtain

Mean of Intercept of regression line- $\log A$

Slop of design curve- $m$

Sample size- $n$

Sum of values- $\sum \log S_{i}$

Mean values- $\overline{\log S}=\frac{\sum \log S_{i}}{n}$

Sum of values- $\sum \log N_{i}$

Mean values- $\overline{\log N}=\frac{\sum N_{i}}{n}$

Estimated mean of Intercept of regression line- $\log \hat{A}$

Estimated value- $\log \hat{N}_{i}=\log \hat{A}-3 \times \log S_{i}$ 


\begin{tabular}{|c|l|l|}
\hline $\begin{array}{c}\text { IIW/IIS } \\
\text { Com XIII-WG1 }\end{array}$ & How to determine a design S-N curve, slope fixed (tolerance limits)? & Sheet 12 \\
\hline
\end{tabular}

Estimated the variance- $\hat{\sigma}^{2}=\frac{\sum_{i=1}^{n}\left(\log N_{i}-\log \hat{N}_{i}\right)^{2}}{f}$

Degree of freedom- $f=n-1$

$\hat{\sigma}=\sqrt{\frac{\sum\left(\log N_{i}-\log \hat{N}_{i}\right)^{2}}{f}}$

One-sided tolerance limit factor- $k$

Design S-N curve: $\log N_{p \%}^{-}=\hat{\mu}-k s$

$$
\begin{aligned}
& \log N_{p \%}^{-}=(\log A-m \log S)-k \hat{\sigma} \sqrt{1+\frac{1}{n}} \\
& \log N_{95 \%}^{-}=(12.2889-3 \log S)-2.65 \times 0.108 \times \sqrt{1+\frac{1}{9}} \\
& \log N_{95 \%}^{-}=11.9869-3 \log S
\end{aligned}
$$

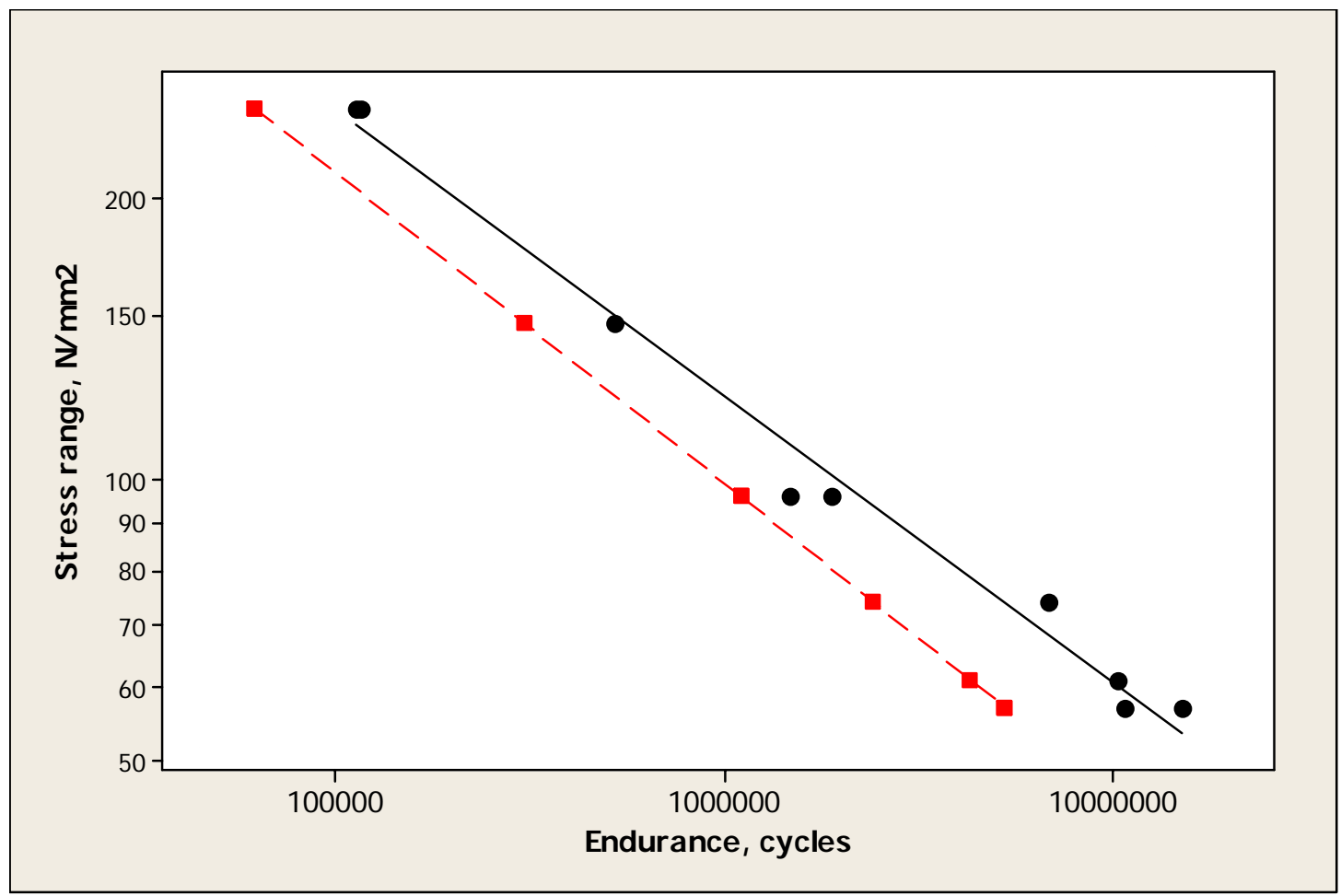

Design curve based on tolerance limits (slope fixed) 


\section{Appendix VII}

\begin{tabular}{|c|c|c|}
\hline $\begin{array}{c}\text { IIW/IIS } \\
\text { Com XIII-WG1 }\end{array}$ & $\begin{array}{c}\text { How to determine a design S-N curve, slop estimated } \\
\text { (tolerance limits)? }\end{array}$ & Sheet 13 \\
\hline
\end{tabular}

\section{General}

\section{Contest}

In Data Sheet12, the instructions for determining a design S-N curve with fixed slope value based on tolerance limits have been illustrated. In the general case where the slope of the regression line is estimated from the data, the tolerance limit factor is different from the previous example.

\section{Principle}

This statement is made on the basis of a sample of $n$ independent observations. A tolerance limit can be regarded as a confidence limit on a prediction limit.

$\log N_{p \%}^{-}=\hat{\mu}-k s$

where: $\hat{\mu}$ is an estimate of the mean $\log$ of the endurance at stress $S$

$s \quad$ is an estimate of the standard deviation of the log of the endurance at stress $S$, based on $f$ degree of freedom

$k$ is a one-sided tolerance limit factor

The involved calculation to determine the one-side tolerance limit factor $k$ is laborious. Mathematically, $k$ is determined by the following equation:

$\operatorname{Pr}\left\{\left(\right.\right.$ noncenral $t$ with $\left.\left.\delta=K_{p} \sqrt{n}\right) \leq k \sqrt{n}\right\}=\gamma$

where: $K_{p}$ is defined by $\frac{1}{\sqrt{2 \pi}} \int_{-\infty}^{K_{p}} \exp \left(-x^{2} / 2\right) d x=P$

If $\delta$ and $f$ are given, the critical value of noncentral $t$-distribution $t_{0}$ can be calculated, then $k$ value can be obtained by equation: $k=\frac{t_{0}}{\sqrt{n}}$.

\section{Condition of application}

- It is assumed that design curves will only be applied to values of $\log S$ that are not far removed from the mean value $\overline{\log S}$. 


\begin{tabular}{|c|c|c|}
\hline $\begin{array}{c}\text { IIW/IIS } \\
\text { Com XIII-WG1 }\end{array}$ & $\begin{array}{c}\text { How to determine a design S-N curve, slop estimated } \\
\text { (tolerance limits)? }\end{array}$ & Sheet 13 \\
\hline
\end{tabular}

\section{Procedure}

\section{1. $\underline{\text { Criteria }}$}

A normal distribution for $\gamma \%=90 \%$, Proportion $95 \%$

The design curves will only be applied to values of $\log S$ that are not far removed from the mean value $\overline{\log S}$

\section{Data and formula}

The correct values for the constants $A$ and $m$ are obtained from the following two equations by using the maximum likelihood method:

$\log A=\overline{\log N}+m \overline{\log S}$

$$
m=\frac{\sum_{i=1}^{n}\left(\log S_{i}-\overline{\log S}\right)\left(\log N_{i}-\overline{\log N}\right)}{\sum_{i=1}^{n}\left(\log S_{i}-\overline{\log S}\right)^{2}}
$$

Sample size- $n$

Degree of freedom- $f=n-2$

Sum of values- $\sum \log S_{i}$

Mean values- $\overline{\log S}=\frac{\sum \log S_{i}}{n}$

Sum of values- $\sum \log N_{i}$

Mean values- $\overline{\log N}=\frac{\sum N_{i}}{n}$

$$
\sum_{i=1}^{n}\left(\log S_{i}-\overline{\log S}\right)\left(\log N_{i}-\overline{\log N}\right)
$$

$\sum_{i=1}^{n}\left(\log S_{i}-\overline{\log S}\right)^{2}$ 


\begin{tabular}{|c|c|c|}
\hline $\begin{array}{c}\text { IIW/IIS } \\
\text { Com XIII-WG1 }\end{array}$ & $\begin{array}{c}\text { How to determine a design S-N curve, slop estimated } \\
\text { (tolerance limits)? }\end{array}$ & Sheet 13 \\
\hline
\end{tabular}

Estimated slope- $\hat{m}$

Estimated mean of Intercept of regression line- $\log \hat{A}$

Estimated value- $\log \hat{N}_{i}=\log \hat{A}-\hat{m} \times \log S_{i}$

Estimated the variance- $\hat{\sigma}^{2}=\frac{\sum_{i=1}^{n}\left(\log N_{i}-\log \hat{N}_{i}\right)^{2}}{f}$

$$
\hat{\sigma}=\sqrt{\frac{\sum\left(\log N_{i}-\log \hat{N}_{i}\right)^{2}}{f}}
$$

Upper critical value of standard normal distribution- $K_{95 \%}$

Value of $\delta=K_{p} \sqrt{n}$

Value of $\eta=\frac{\delta}{\sqrt{2 f}}\left(1+\frac{\delta^{2}}{2 f}\right)^{-1 / 2}$

Value of $\lambda$ based on $\eta, f$

Critical values of the Noncentral $t$-distribution- $t_{0}=\frac{\delta+\lambda\left(1+\frac{\delta^{2}}{2 f}-\frac{\lambda^{2}}{2 f}\right)}{1-\left(\lambda^{2} / 2 f\right)}$

According to $\operatorname{Pr}($ noncenral $t \leq k \sqrt{n})=\gamma \%$

One-sided tolerance limit factor- $k=t_{0} / \sqrt{n}$

Design S-N curve: $\log N_{p \%}^{ \pm}=(\log A-m \log S) \pm k \hat{\sigma} \sqrt{1+\frac{1}{n}}$ 


\begin{tabular}{|c|c|c|}
\hline $\begin{array}{c}\text { IIW/IIS } \\
\text { Com XIII-WG1 }\end{array}$ & $\begin{array}{c}\text { How to determine a design S-N curve, slop estimated } \\
\text { (tolerance limits)? }\end{array}$ & Sheet 13 \\
\hline
\end{tabular}

\section{Example}

\section{Data and formula}

\section{Samples data}

$n=18$

\begin{tabular}{|c|c|c|c|}
\hline Stress range & cycles to failure & Stress range & cycles to failure \\
\hline 147 & 521,382 & 74 & $6,195,271$ \\
\hline 96 & $1,879,752$ & 74 & $4,310,000$ \\
\hline 250 & 115,816 & 136 & $1,015,824$ \\
\hline 61 & $10,204,041$ & 136 & $1,580,669$ \\
\hline 57 & $14,910,395$ & 53 & $11,541,520$ \\
\hline 57 & $10,646,018$ & 136 & $1,133,000$ \\
\hline 96 & $1,475,769$ & 74 & $3,675,000$ \\
\hline 250 & 112,910 & 53 & $22,082,998$ \\
\hline 74 & $6,766,000$ & 53 & $7,154,785$ \\
\hline
\end{tabular}

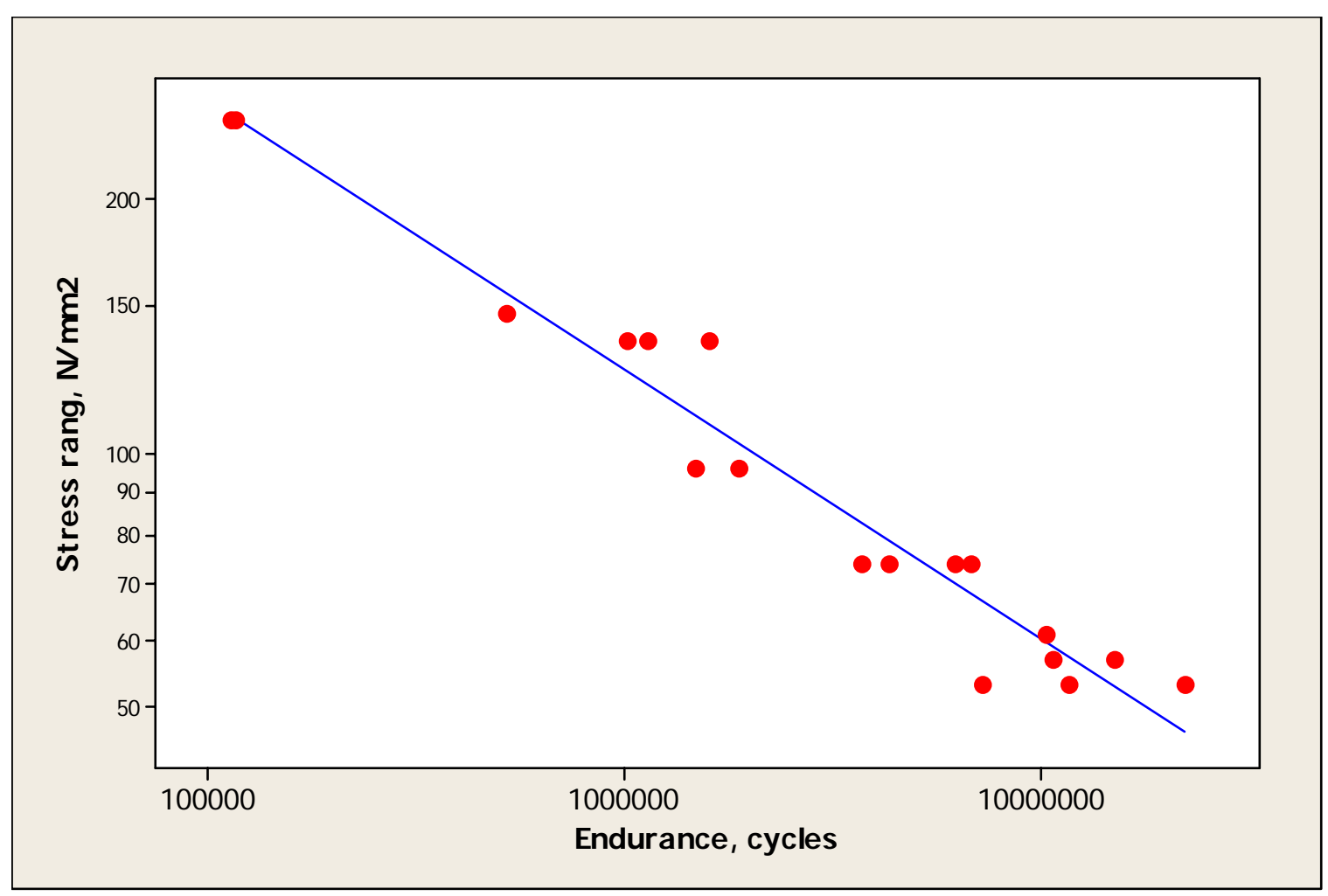

Linear regression S-N curve 


\begin{tabular}{|c|c|c|}
\hline $\begin{array}{c}\text { IIW/IIS } \\
\text { Com XIII-WG1 }\end{array}$ & $\begin{array}{c}\text { How to determine a design S-N curve, slop estimated } \\
\text { (tolerance limits)? }\end{array}$ & Sheet 13 \\
\hline
\end{tabular}

\section{Calculation}

Proportion 95\%

The correct values for the constants $A$ and $k$ are obtained from the following two equations by using the maximum likelihood method:

$$
\begin{aligned}
& \log \hat{A}=\overline{\log N}+m \overline{\log S} \\
& \hat{m}=-\frac{\sum_{i=1}^{n}\left(\log S_{i}-\overline{\log S}\right)\left(\log N_{i}-\overline{\log N}\right)}{\sum_{i=1}^{n}\left(\log S_{i}-\overline{\log S}\right)^{2}}
\end{aligned}
$$

Sample size- $n$

Degree of freedom- $f=n-2$

Sum of values- $\sum \log S_{i}$

Mean values- $\overline{\log S}=\frac{\sum \log S_{i}}{n}$

Sum of values- $\sum \log N_{i}$

Mean values- $\overline{\log N}=\frac{\sum N_{i}}{n}$

$\sum_{i=1}^{n}\left(\log S_{i}-\overline{\log S}\right)\left(\log N_{i}-\overline{\log N}\right)$

$\sum_{i=1}^{n}\left(\log S_{i}-\overline{\log S}\right)^{2}$

Estimated slope- $\hat{m}$

Estimated mean of Intercept of regression line- $\log \hat{A}$

Estimated value- $\log \hat{N}_{i}=\log \hat{A}-\hat{m} \times \log S_{i}$ 


\begin{tabular}{|c|c|c|}
\hline $\begin{array}{c}\text { IIW/IIS } \\
\text { Com XIII-WG1 }\end{array}$ & $\begin{array}{c}\text { How to determine a design S-N curve, slop estimated } \\
\text { (tolerance limits)? }\end{array}$ & Sheet 13 \\
\hline
\end{tabular}

Estimated the variance- $\hat{\sigma}^{2}=\frac{\sum_{i=1}^{n}\left(\log N_{i}-\log \hat{N}_{i}\right)^{2}}{f}$

$$
\hat{\sigma}=\sqrt{\frac{\sum\left(\log N_{i}-\log \hat{N}_{i}\right)^{2}}{f}}
$$

Upper critical value of standard normal distribution- $K_{95 \%}$

Value of $\delta=K_{95 \%} \sqrt{n}$

Value of $\eta=\frac{\delta}{\sqrt{2 f}}\left(1+\frac{\delta^{2}}{2 f}\right)^{-1 / 2}$

Value of $\lambda$ based on $\eta, f$

Critical values of the Noncentral $t$-distribution- $t_{0}=\frac{\delta+\lambda\left(1+\frac{\delta^{2}}{2 f}-\frac{\lambda^{2}}{2 f}\right)}{1-\left(\lambda^{2} / 2 f\right)}$

According to $\operatorname{Pr}($ noncenral $t \leq k \sqrt{n})=90 \%$

One-sided tolerance limit factor- $k=t_{0} / \sqrt{n}$

Design S-N curve: $\log N_{p \%}^{ \pm}=(\log A-m \log S)-k \hat{\sigma} \sqrt{1+\frac{1}{n}}$

$$
\begin{aligned}
& \log N_{95 \%}^{ \pm}=(12.2729-2.979 \log S)-2.575 \times 0.1522 \sqrt{1+\frac{1}{18}} \\
& \log N_{95 \%}^{ \pm}=11.8699-2.979 \log S
\end{aligned}
$$




\begin{tabular}{|c|c|c|}
\hline $\begin{array}{c}\text { IIW/IIS } \\
\text { Com XIII-WG1 }\end{array}$ & $\begin{array}{c}\text { How to determine a design S-N curve, slop estimated } \\
\text { (tolerance limits)? }\end{array}$ & Sheet 13 \\
\hline
\end{tabular}

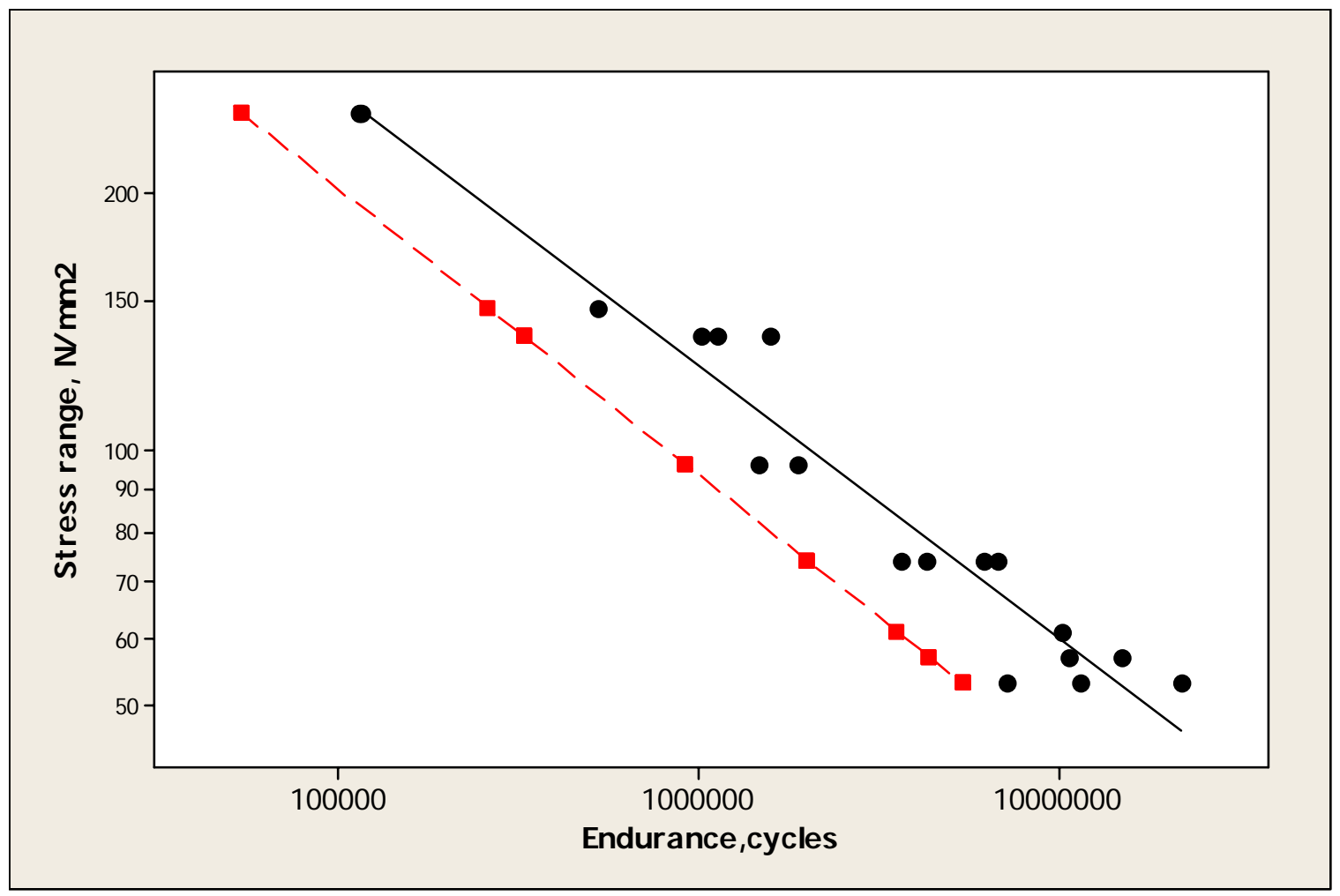

Design curve based on tolerance limits (slope estimated) 


\section{Appendix VIII}

\begin{tabular}{|c|c|c|}
$\begin{array}{c}\text { IIW/IIS } \\
\text { Com XIII-WG1 }\end{array}$ & Are 2 experimental design S-N curves statistically equivalent? & Sheet 15 \\
\hline
\end{tabular}

\section{General}

\section{Contest}

The problem is likely to be of interest where the two data sets have been collected under different conditions, but are expected to give comparable fatigue performance. The following methods are used to justify the conclusion that there is a significant difference between them.

\section{Principle}

Whether two experimental design S-N curves are statistically equivalent can be determined by using a hypothesis test. Because the design curve is established by adopting characteristic values lay a certain number of standard deviations below the mean S-N curve, the question can be solved to determine the statistical equivalence of the mean S-N curve.

A lower significance level is often used for each individual hypothesis test. For instance, a significance level of $1.7 \%$ for each individual test would roughly correspond to a 5\% significance level for the composite hypothesis.

\section{Condition of application}

Design curves will only be applied to values of $\log S$ that are not far removed for the mean value. The necessary data to perform the equivalence verification are the following:

- The two data sets, $\sigma 1_{i}, n 1$ values and $\sigma 2_{i}, n 2$ values

It is assumed here that both sets of S-N data are exact data

If the data sets do not follow a normal distribution, the number of $n 1$ and $n 2$ shall be greater or equal to 30 .

- The estimated means of the 2 data sets

- The estimated variances of the 2 data sets 


\begin{tabular}{|c|c|c|}
\hline $\begin{array}{c}\text { IIW/IIS } \\
\text { Com XIII-WG1 }\end{array}$ & Are 2 experimental design S-N curves statistically equivalent? & Sheet 15 \\
\hline
\end{tabular}

\section{Procedure}

\section{Criteria}

The one of applied tests is the two-sided Snedecor F-test with unpaired samples.

The selected level of risk is set to $\alpha, \alpha \%$ probability to reject a correct hypothesis.

\section{Data and formula}

\section{Test that residual standard deviation are consistent}

The correct values for the constants $A$ and $m$ are obtained from the following two equations by using the maximum likelihood method:

$$
\begin{aligned}
& \log A=\overline{\log N}+m \overline{\log S} \\
& m=\frac{\sum_{i=1}^{n}\left(\log S_{i}-\overline{\log S}\right)\left(\log N_{i}-\overline{\log N}\right)}{\sum_{i=1}^{n}\left(\log S_{i}-\overline{\log S}\right)^{2}}
\end{aligned}
$$

Sample size- $n_{i}$

Sum of values- $\sum \log N_{i}$

Mean value- $\overline{\log N_{i}}=\frac{\sum \log N_{i}}{n_{i}}$

Sum of values- $\sum \log S_{i}$

Mean value- $\overline{\log S_{i}}=\frac{\sum \log S_{i}}{n_{i}}$

$\sum_{i=1}^{n}\left(\log S_{i, i}-\overline{\log S_{i}}\right)^{2}$

Estimated value of the slop- $\hat{m}$

Estimated value of the intercept- $\log \hat{A}$

Degree of freedom- $f_{i}=n_{i}-2$

Estimated value- $\log \hat{N}_{i}=\log \hat{A}-\hat{m} \log S_{i}$ 


\begin{tabular}{|c|c|c|}
\hline $\begin{array}{c}\text { IIW/IIS } \\
\text { Com XIII-WG1 }\end{array}$ & Are 2 experimental design S-N curves statistically equivalent? & Sheet 15 \\
\hline
\end{tabular}

Estimated variances- $\hat{\sigma}_{i}^{2}=\frac{\sum\left(\log N_{i}-\log \hat{N}_{i}\right)^{2}}{f}$

Variance ratio- $R=\frac{\hat{\sigma}_{i}^{2}}{\hat{\sigma}_{j}^{2}}$

Critical value for one-sided Snedecor F-distribution- $F\left(1-\alpha, f_{i}, f_{j}\right)$

Criteria- $C r=R-F\left(1-\alpha, f_{i}, f_{j}\right)$

Conclusion:

If $\mathrm{Cr}>0$ the hypothesis of equivalence has to be rejected

\section{Test that the intercepts of the two S-N Consistent}

Two-sided percentage point of Student'st distribution- $t\left(1-\alpha, n_{1}+n_{2}-4\right)$

An estimate of the common variance of the two samples- $\sigma_{e}$

$$
\begin{aligned}
& \hat{\sigma}_{1}^{2}=\ldots \ldots \ldots \cdot \cdots \\
& \sigma_{e}^{2}=\frac{f_{1} \hat{\sigma}_{1}^{2}+f_{2} \hat{\sigma}_{2}^{2}}{f_{1}+f_{2}}
\end{aligned}
$$

Estimated intercepts of the regression lines- $\overline{\log A_{i}}$

Mean difference of estimated intercepts- $X=\left|\overline{\log A_{1}}-\overline{\log A_{2}}\right|$

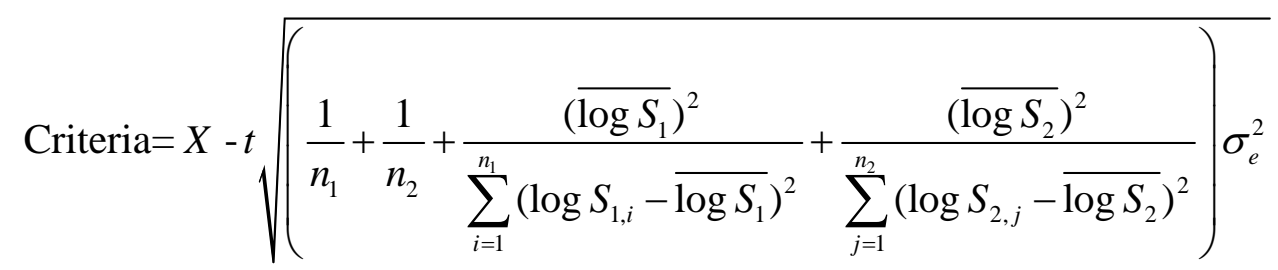

Conclusion:

If $\mathrm{Cr}>0$ the hypothesis of equivalence has to be rejected 


\begin{tabular}{|c|c|c|}
\hline $\begin{array}{c}\text { IIW/IIS } \\
\text { Com XIII-WG1 }\end{array}$ & Are 2 experimental design S-N curves statistically equivalent? & Sheet 15 \\
\hline
\end{tabular}

Testing that the slopes of the two S-N curves are consistent

Estimated slopes of the regression lines- $m_{i}$

Difference of estimated slopes- $T=\left|m_{1}-m_{2}\right|$

Criteria $=T-t \sqrt{\left(\frac{1}{\sum_{i=1}^{n_{1}}\left(\log S_{1, i}-\overline{\log S_{1}}\right)^{2}}+\frac{1}{\sum_{j=1}^{n_{2}}\left(\log S_{2, j}-\overline{\log S_{2}}\right)^{2}}\right) \sigma_{e}^{2}}$

Conclusion:

If $\mathrm{Cr}>0$ the hypothesis of equivalence has to be rejected

\section{Conclusion}

If one of above hypothesis is rejected, the composite hypothesis can not be accepted. 


\begin{tabular}{|c|c|c|}
\hline $\begin{array}{c}\text { IIW/IIS } \\
\text { Com XIII-WG1 }\end{array}$ & Are 2 experimental design S-N curves statistically equivalent? & Sheet 15 \\
\hline
\end{tabular}

\section{Example}

\section{Data and formula}

\section{Samples data}

No.1 $n_{1}=14$

\begin{tabular}{|c|c|}
\hline Stress range & Cycles to failure \\
\hline 147 & 521382 \\
\hline 96 & 1879752 \\
\hline 250 & 115816 \\
\hline 61 & 10204041 \\
\hline 57 & 14910395 \\
\hline 57 & 10646018 \\
\hline 96 & 1475769 \\
\hline 250 & 112910 \\
\hline 74 & 6766000 \\
\hline 74 & 6195271 \\
\hline 74 & 4310000 \\
\hline 136 & 1015824 \\
\hline 136 & 1580669 \\
\hline 53 & 11541520 \\
\hline & \\
\hline
\end{tabular}

No. $2 \quad n_{2}=15$

\begin{tabular}{|c|c|}
\hline Stress range & Cycles to failure \\
\hline 136 & 1133000 \\
\hline 74 & 3675000 \\
\hline 53 & 22082998 \\
\hline 53 & 7154785 \\
\hline 136 & 664000 \\
\hline 75 & 3816199 \\
\hline 136 & 787894 \\
\hline 136 & 689973 \\
\hline 147 & 958318 \\
\hline 53 & 13475000 \\
\hline 53 & 20511538 \\
\hline 265 & 95982 \\
\hline 54 & 19048838 \\
\hline 176 & 605721 \\
\hline 74 & 9232000 \\
\hline
\end{tabular}

\section{Calculation}

The risk level is fixed to $\alpha=1.7 \%$

\section{Test that residual standard deviation are consistent}

The correct values for the constants $A$ and $m$ are obtained from the following two equations by using the maximum likelihood method:

$\log A=\overline{\log N}+m \overline{\log S}$

$m=\frac{\sum_{i=1}^{n}\left(\log S_{i}-\overline{\log S}\right)\left(\log N_{i}-\overline{\log N}\right)}{\sum_{i=1}^{n}\left(\log S_{i}-\overline{\log S}\right)^{2}}$ 


\begin{tabular}{|c|c|c|}
\hline $\begin{array}{c}\text { IIW/IIS } \\
\text { Com XIII-WG1 }\end{array}$ & Are 2 experimental design S-N curves statistically equivalent? & Sheet 15 \\
\hline
\end{tabular}

Sample No.1

Sample No.2

Sample size- $n_{i}$

14

15

Sum of values- $\sum \log N_{i}$

89.011

96.390

Mean value- $\overline{\log N_{i}}=\frac{\sum \log N_{i}}{n_{i}}$

Sum of values- $\sum \log S_{i}$

Mean value- $\overline{\log S_{i}}=\frac{\sum \log S_{i}}{n_{i}}$

$\sum_{i=1}^{n}\left(\log S_{i, i}-\overline{\log S_{i}}\right)^{2}$

0.7543

Estimated value of the slop- $\hat{m}$

3.062

3.008

Estimated value of the intercept- $\log \hat{A}$

Degree of freedom- $f_{i}=n_{i}-2$

Estimated value- $\log \hat{N}_{i}=\log \hat{A}-\hat{m} \log S_{i}$

Estimated variances- $\hat{\sigma}_{i}^{2}=\frac{\sum\left(\log N_{i}-\log \hat{N}_{i}\right)^{2}}{f} \quad 0.0171$

Variance ratio- $R=\frac{\hat{\sigma}_{i}^{2}}{\hat{\sigma}_{j}^{2}}$

Critical value for one-sided Snedecor F-distribution- $F\left(1-\alpha, f_{i}, f_{j}\right)$

Criteria- $C r=R-F\left(1-\alpha, f_{i}, f_{j}\right)$

Conclusion:

As $\mathrm{Cr}<0$ the hypothesis of equivalence has no reason to be rejected and therefore the residual standard deviations are statistically equivalent. 


\begin{tabular}{|c|c|c|}
\hline $\begin{array}{c}\text { IIW/IIS } \\
\text { Com XIII-WG1 }\end{array}$ & Are 2 experimental design S-N curves statistically equivalent? & Sheet 15 \\
\hline
\end{tabular}

\section{Test that the intercepts of the two S-N Consistent}

Two-sided percentage point of Student's distribution- $t\left(1-\alpha, n_{1}+n_{2}-4\right)$

An estimate of the common variance of the two samples- $\sigma_{e}$

$$
\begin{array}{ll}
\hat{\sigma}_{1}^{2}=0.0171 & \hat{\sigma}_{2}^{2}=0.0283 \\
\sigma_{e}^{2}=\frac{f_{1} \hat{\sigma}_{1}^{2}+f_{2} \hat{\sigma}_{2}^{2}}{f_{1}+f_{2}} &
\end{array}
$$

Estimated intercepts of the regression lines- $\overline{\log A_{i}}$

Mean difference of estimated intercepts- $X=\left|\overline{\log A_{1}}-\overline{\log A_{2}}\right|$

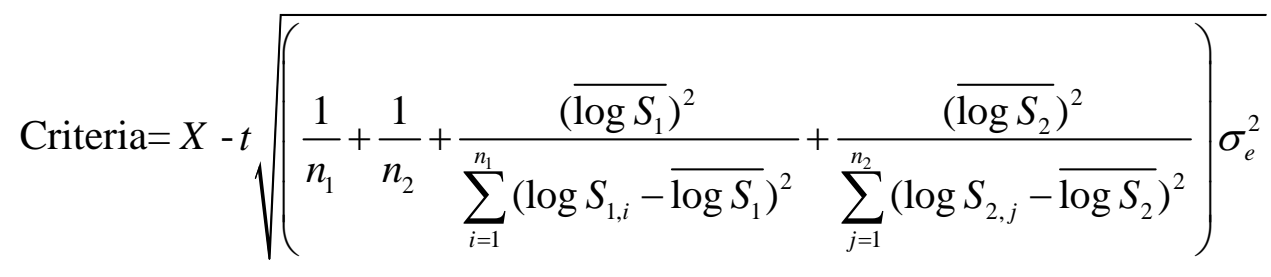

$=0.079-2.5572 \times \sqrt{\left(\frac{1}{14}+\frac{1}{15}+\frac{3.95}{0.6714}+\frac{3.90}{0.7543}\right) 0.0382}$

$=0.079-1.672=-1.593$

Conclusion:

As $\mathrm{Cr}<0$ the hypothesis of equivalence has no reason to be rejected

\section{Testing that the slopes of the two S-N curves are consistent}

Estimated slopes of the regression lines- $m_{i}$

Difference of estimated slopes- $T=\left|m_{1}-m_{2}\right|$ 


\begin{tabular}{|c|c|c|}
\hline $\begin{array}{c}\text { IIW/IIS } \\
\text { Com XIII-WG1 }\end{array}$ & Are 2 experimental design S-N curves statistically equivalent? & Sheet 15 \\
\hline
\end{tabular}

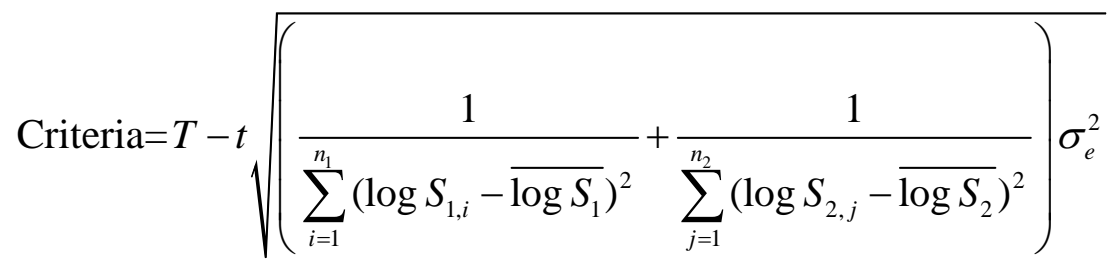

Criteria $=0.054-2.5572 \sqrt{\left(\frac{1}{0.6714}+\frac{1}{0.7543}\right) 0.0382}$

Criteria $=0.054-0.839=-0.785$

Conclusion:

As $C r<0$ the hypothesis of equivalence has no reason to be rejected

\section{Conclusion}

The above three individual null hypothesizes with a significance level $1.7 \%$ are accepted. Now, we can draw the conclusion this composite hypothesis with a significance level $5 \%$ is accepted. 


\begin{tabular}{|c|c|c|}
\hline \multicolumn{3}{|l|}{ Appendix IX } \\
\hline $\begin{array}{c}\text { IIW/IIS } \\
\text { Com XIII-WG1 }\end{array}$ & $\begin{array}{l}\text { How to determine the degree of improvement produced by a } \\
\text { post-weld treatment process? }\end{array}$ & Sheet 16 \\
\hline
\end{tabular}

\section{General}

\section{Context}

A post-weld treatment is implemented to remove welding residual stresses in order to pursue improvement in fatigue behavior. Commonly used residual stress methods are hammer peening, wire bundle, shot peening and ultrasonic peening.

The magnitude of improvement depends primarily on the joint severity and base material. The pose-weld treatment can be regarded as a means of improving the fatigue strength of welded joints.

\section{Principle}

The term fatigue class (FAT) indicates the characteristic stress range, which gives a fatigue life of two million cycles at $95 \%$ survival probability. The data is able to be evaluated according to the statistical methods outlined by the Huther.

In order to prove that a process has been improved, we must measure the process capability before and after improvements are implemented. The difference of FAT between the as-weld and post-weld indicates the degree of improvement produced by the post-weld treatment.

\section{Condition of application}

- It is noticed that the benefit of peening of steel components can only be claimed for details in design Class FAT 90 or lower in the IIW notation for S-N curves.

- The effect of specimen thickness on fatigue strength was only slight.

\section{Conclusion}

The conclusion is able to be drawn after the comparison based on the statistical analysis. Generally, there is a definite increase in the fatigue life of the as-weld versus post-weld in these tests. 


\begin{tabular}{|c|c|c|}
\hline $\begin{array}{c}\text { IIW/IIS } \\
\text { Com XIII-WG1 }\end{array}$ & $\begin{array}{c}\text { How to determine the degree of improvement produced by a } \\
\text { post-weld treatment process? }\end{array}$ & Sheet 16 \\
\hline
\end{tabular}

\section{Procedure}

\section{Criteria}

A failure probability of 97.5 percent can be used in the standard since the results are based on a large number of test pieces.

If the sample size is small, characteristic fatigue strength is $F A T_{95 \%}$ and not $F A T_{97.5 \%}$

\section{Data and formula}

Sample size- $n$

Slop of curve- $m$

Calculate the $F A T_{95 \%}$ by following equations:

$\Delta \sigma_{i}^{m} \cdot N_{i}=C_{i}=F A T^{m} \cdot 2000000$

Sum of values- $\sum \log C_{i}$

Mean fatigue capacity- $\log C_{50 \%}=\frac{\sum \log C_{i}}{n}$

Standard deviation $s=\sqrt{\frac{\sum\left(\log C_{i}-\log C_{50 \%}\right)^{2}}{n-1}}$

$\log C_{95 \%}=\log C_{50 \%}-s\left(1.64+\frac{1.15}{\sqrt{n}}\right)$

Characteristic Fatigue capacity- FAT $T_{95 \%}=\sqrt[m]{\frac{C_{95 \%}}{2000000}}$ 


\begin{tabular}{|c|c|c|}
\hline $\begin{array}{c}\text { IIW/IIS } \\
\text { Com XIII-WG1 }\end{array}$ & $\begin{array}{c}\text { How to determine the degree of improvement produced by a } \\
\text { post-weld treatment process? }\end{array}$ & Sheet 16 \\
\hline
\end{tabular}

\section{Example 1}

Note: The slope of the S-N curve $m$ is fixed.

\section{Data and formula}

Samples data No.1

\begin{tabular}{|c|c|c|c|c|c|c|}
\hline & $\mathrm{t}$ & $\mathrm{R}$ & $\Delta \sigma$ & $\mathrm{N}$ & $\mathrm{FAT}(\mathrm{m}=3)$ & Observation \\
\hline AW-11 & 5 & 0.1 & 180 & 599377 & 120 & $\mathrm{WT}$ \\
\hline AW-12 & 5 & 0.1 & 221 & 422755 & 131 & $\mathrm{WT}$ \\
\hline AW-13 & 5 & 0.1 & 127 & 2173795 & 131 & WT \\
\hline AW-14 & 5 & 0.1 & 132 & 1313035 & 115 & WT \\
\hline AW-15 & 5 & 0.1 & 204 & 480284 & 127 & WT \\
\hline UIT-1 & 5 & 0.1 & 349 & 596082 & 233 & UITG \\
\hline UIT-2 & 5 & 0.1 & 318 & 310170 & 171 & UITG \\
\hline UIT-3 & 5 & 0.1 & 324 & 620074 & 219 & UITG \\
\hline UIT-4 & 5 & 0.1 & 327 & 505913 & 207 & UITG \\
\hline UIT-5 & 5 & 0.1 & 318 & 781200 & 232 & UITG \\
\hline
\end{tabular}

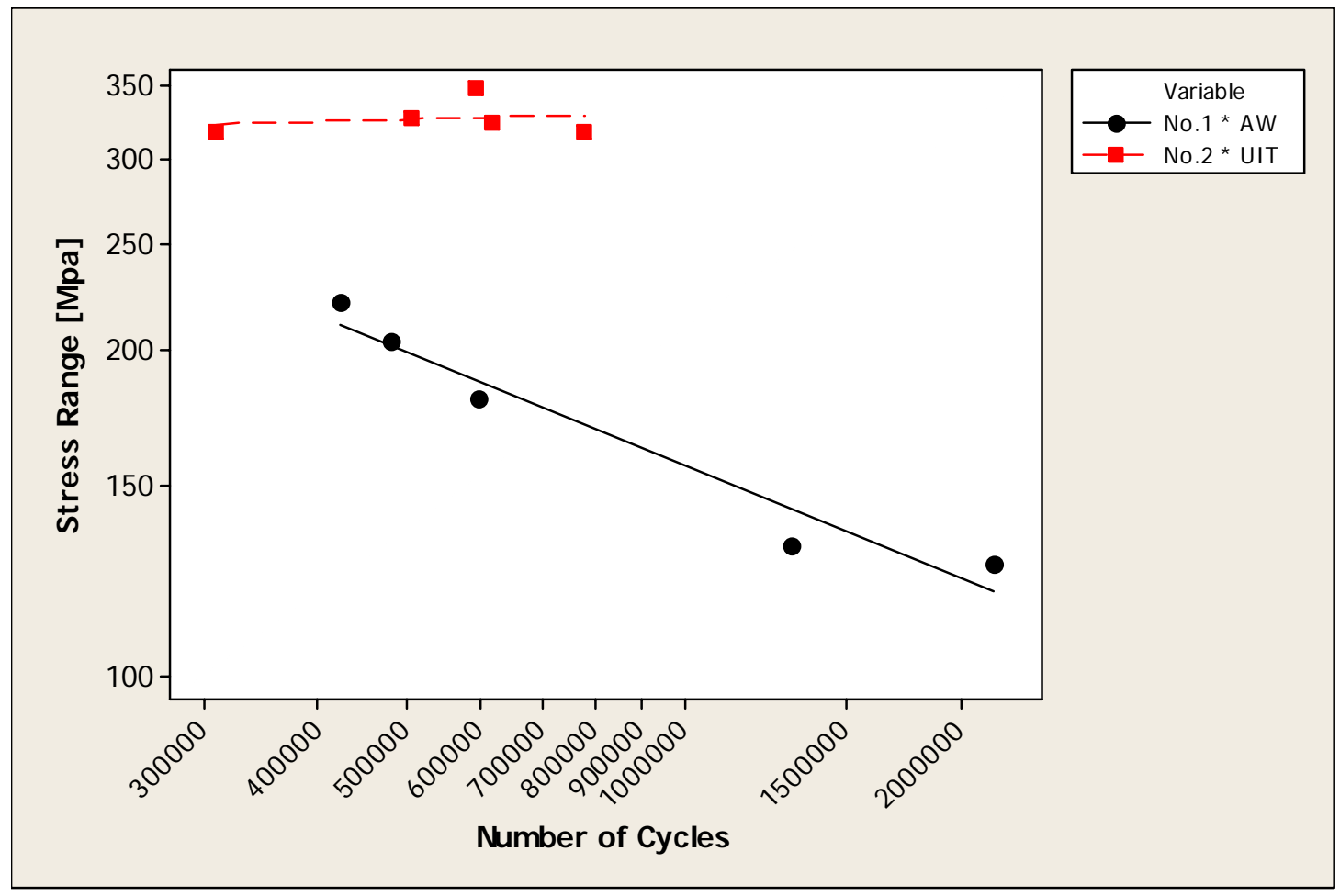

The comparison between as-welded and UI-treated test series, $t=5 \mathrm{~mm}, R=0.1$ 


\begin{tabular}{|c|c|c|}
\hline $\begin{array}{c}\text { IIW/IIS } \\
\text { Com XIII-WG1 }\end{array}$ & $\begin{array}{c}\text { How to determine the degree of improvement produced by a } \\
\text { post-weld treatment process? }\end{array}$ & Sheet 16 \\
\hline
\end{tabular}

\section{Calculation}

No.1 AW 5

3
No.1 UIT

Sample size- $n$

Slop of curve- $m$

Calculate the $F A T_{95 \%}$ by following equations: $\Delta \sigma_{i}^{m} \cdot N_{i}=C_{i}=F A T^{m} \cdot 2000000$

Sum of values- $\sum \log C_{i}$

62.9418

66.3745

Mean fatigue capacity- $\log C_{50 \%}=\frac{\sum \log C_{i}}{n}$

12.5884

13.2749

Standard deviation- $s=\sqrt{\frac{\sum\left(\log C_{i}-\log C_{50 \%}\right)^{2}}{n-1}}$

0.07566

0.16704

$\log C_{95 \%}=\log C_{50 \%}-s\left(1.64+\frac{1.15}{\sqrt{n}}\right)$

12.4254

Characteristic Fatigue capacity- $F A T_{95 \%}=\sqrt[m]{\frac{C_{95 \%}}{2000000}}$

110.0162

160.1978 


\begin{tabular}{|c|c|c|}
\hline $\begin{array}{c}\text { IIW/IIS } \\
\text { Com XIII-WG1 }\end{array}$ & $\begin{array}{c}\text { How to determine the degree of improvement produced by a } \\
\text { post-weld treatment process? }\end{array}$ & Sheet 16 \\
\hline
\end{tabular}

\section{Example 2}

\section{Data and formula}

Samples data No.2

\begin{tabular}{|c|c|c|c|c|c|c|}
\hline & $t$ & $R$ & $\Delta \sigma$ & $N$ & FAT $(m=3)$ & Observation \\
\hline AW-28 & 8 & 0.1 & 193 & 485897 & 120 & WT \\
\hline AW-29 & 8 & 0.1 & 193 & 640024 & 132 & WT \\
\hline AW-30 & 8 & 0.1 & 161 & 1257193 & 138 & WT \\
\hline AW-31 & 8 & 0.1 & 180 & 1091393 & 147 & WT \\
\hline AW-32 & 8 & 0.1 & 169 & 1199013 & 143 & WT \\
\hline UIT-18 & 8 & 0.1 & 288 & 1902884 & 283 & UITG \\
\hline UIT-19 & 8 & 0.1 & 369 & 441958 & 223 & UITG \\
\hline UIT-20 & 8 & 0.1 & 383 & 407610 & 225 & UITG \\
\hline UIT-21 & 8 & 0.1 & 335 & 588203 & 223 & UITG \\
\hline UIT-22 & 8 & 0.1 & 338 & 1892369 & 332 & UITG \\
\hline
\end{tabular}

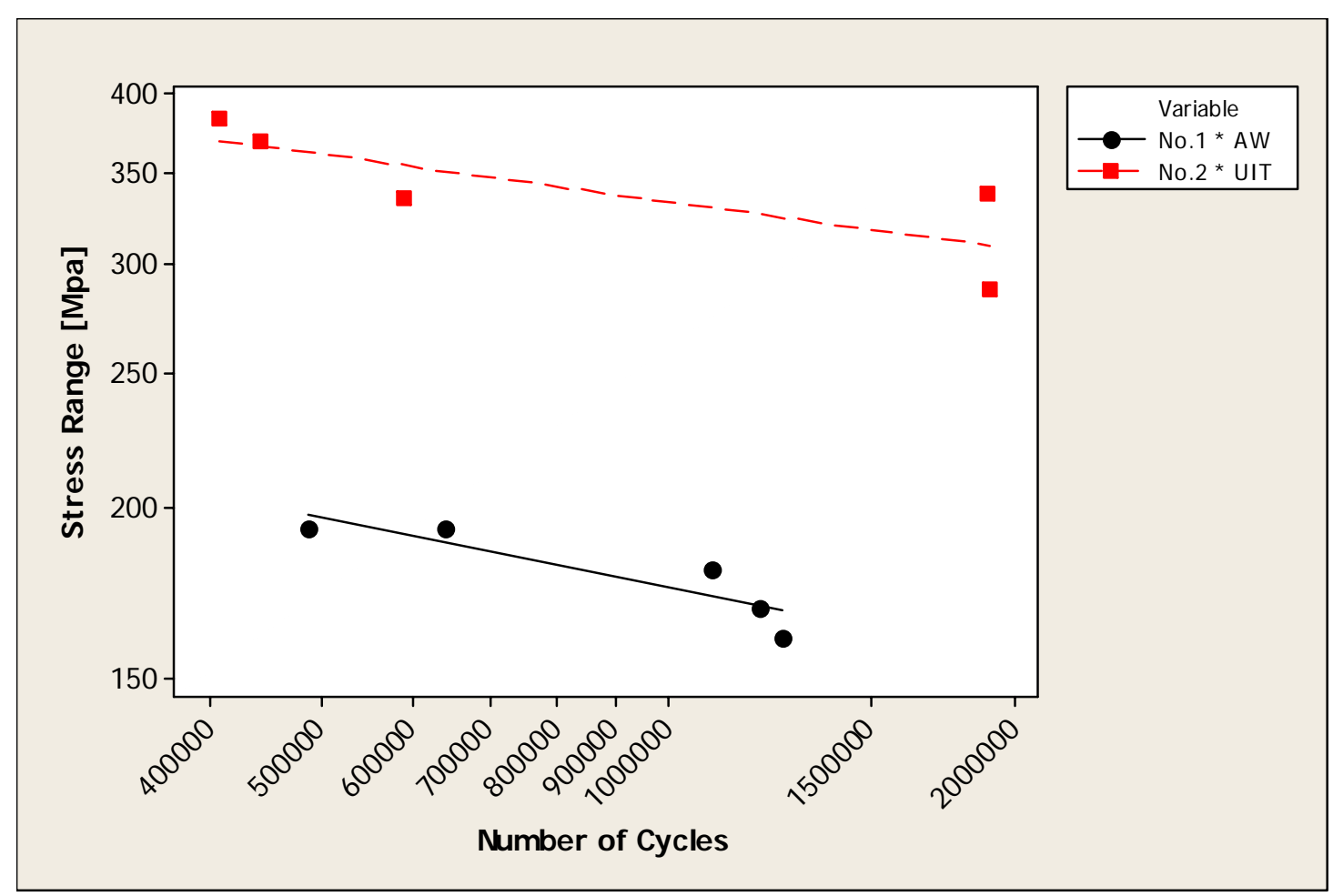

The comparison between as-welded and UI-treated test series, $t=8 \mathrm{~mm}, R=0.1$ 


\begin{tabular}{|c|c|c|}
\hline $\begin{array}{c}\text { IIW/IIS } \\
\text { Com XIII-WG1 }\end{array}$ & $\begin{array}{c}\text { How to determine the degree of improvement produced by a } \\
\text { post-weld treatment process? }\end{array}$ & Sheet 16 \\
\hline
\end{tabular}

\section{Calculation}

No.2 AW No.2UIT

Sample size- $n$

5

5

Slop of curve- $m$

3

3

Calculate the $F A T_{95 \%}$ by following equations: $\quad \Delta \sigma_{i}^{m} \cdot N_{i}=C_{i}=F A T^{m} \cdot 2000000$

Sum of values- $\sum \log C_{i}$

63.4923

67.5723

Mean fatigue capacity- $\log C_{50 \%}=\frac{\sum \log C_{i}}{n}$

Standard deviation- $s=\sqrt{\frac{\sum\left(\log C_{i}-\log C_{50 \%}\right)^{2}}{n-1}} \quad 0.1013 \quad 0.2364$

$\log C_{95 \%}=\log C_{50 \%}-s\left(1.64+\frac{1.15}{\sqrt{n}}\right)$

Characteristic Fatigue capacity- $F A T_{95 \%}=\sqrt[m]{\frac{C_{95 \%}}{2000000}}$

\section{Calculation}

Table: Comparison fatigue classes FAT $_{95 \%}$ between welded and treated series, $\mathbf{m}$ is fixed

\begin{tabular}{|c|c|c|c|}
\hline & $\begin{array}{c}\text { post-weld } \\
\text { treatment }\end{array}$ & as-weld treatment & Post-we1d/as-we1d \\
\hline FAT95\%(t=5mm) & 160 & 110 & 1.45 \\
\hline FAT95\%(t=8mm) & 172 & 115 & 1.50 \\
\hline FAT95\%(8mm)/FAT95\%(5mm) & 1.075 & 1.045 & \\
\hline
\end{tabular}

The fatigue strength of post-weld welds is $45 \%-50 \%$ higher than the fatigue class for as-welded specimens. The fatigue strength of $8 \mathrm{~mm}$ thick specimen is higher than $5 \mathrm{~mm}$ thick specimen. It also turns out that the thickness of specimen does not affect the degree of improvement in fatigue strength greatly. 


\begin{tabular}{|c|c|c|}
\hline $\begin{array}{c}\text { IIW/IIS } \\
\text { Com XIII-WG1 }\end{array}$ & $\begin{array}{c}\text { How to determine the degree of improvement produced by a } \\
\text { post-weld treatment process? }\end{array}$ & Sheet 16 \\
\hline
\end{tabular}

\section{Example 3}

Note: The slope of the S-N curve $\mathrm{m}$ is estimated.

\section{Data and formula}

\section{Samples data No.2}

\begin{tabular}{|c|c|c|c|c|c|c|}
\hline & $t$ & $R$ & $\Delta \sigma$ & $N$ & FAT $(m=3)$ & Observation \\
\hline AW-28 & 8 & 0.1 & 193 & 485897 & 120 & WT \\
\hline AW-29 & 8 & 0.1 & 193 & 640024 & 132 & WT \\
\hline AW-30 & 8 & 0.1 & 161 & 1257193 & 138 & WT \\
\hline AW-31 & 8 & 0.1 & 180 & 1091393 & 147 & WT \\
\hline AW-32 & 8 & 0.1 & 169 & 1199013 & 143 & WT \\
\hline UIT-18 & 8 & 0.1 & 288 & 1902884 & 283 & UITG \\
\hline UIT-19 & 8 & 0.1 & 369 & 441958 & 223 & UITG \\
\hline UIT-20 & 8 & 0.1 & 383 & 407610 & 225 & UITG \\
\hline UIT-21 & 8 & 0.1 & 335 & 588203 & 223 & UITG \\
\hline UIT-22 & 8 & 0.1 & 338 & 1892369 & 332 & UITG \\
\hline
\end{tabular}

\section{Calculation}

The correct values for the constants $A$ and $m$ are obtained by using least squares regression method:

$$
\begin{aligned}
& Y=A-m X \\
& Y=\log N \\
& A=\frac{\sum y_{i}}{n}+m \frac{\sum x_{i}}{n} \\
& m=-\frac{n \sum x_{i} y_{i}-\sum x_{i} \sum y_{i}}{n \sum x_{i}^{2}-\left(\sum x_{i}\right)^{2}} \\
& m_{\text {AW }}=\frac{5 \times 66.888-334.555}{5 \times 25.367-126.812} \\
& m_{A W}=4.754 \\
& m_{U I T}=\frac{5 \times 74.8701-374.609}{5 \times 32.082-160.366} \\
& m_{U I T}=5.63
\end{aligned}
$$




\begin{tabular}{|c|c|c|}
\hline $\begin{array}{c}\text { IIW/IIS } \\
\text { Com XIII-WG1 }\end{array}$ & $\begin{array}{c}\text { How to determine the degree of improvement produced by a } \\
\text { post-weld treatment process? }\end{array}$ & Sheet 16 \\
\hline
\end{tabular}

No.2 AW No.2 UIT

Sample size- $n$

5

5

Slop of curve- $m$

4.754

5.630

Calculate the $F A T_{95 \%}$ by following equations: $\quad \Delta \sigma_{i}^{m} \cdot N_{i}=C_{i}=F A T^{m} \cdot 2000000$

Sum of values- $\sum \log C_{i}$

100.878

Mean fatigue capacity- $\log C_{50 \%}=\frac{\sum \log C_{i}}{n}$

20.1755

Standard deviation- $s=\sqrt{\frac{\sum\left(\log C_{i}-\log C_{50 \%}\right)^{2}}{n-1}}$

$\log C_{95 \%}=\log C_{50 \%}-s\left(1.64+\frac{1.15}{\sqrt{n}}\right)$

Characteristic Fatigue capacity- $F A T_{95 \%}=\sqrt[m]{\frac{C_{95 \%}}{2000000}}$ 138.0658 244.196

Table Comparison fatigue classes FAT $_{95}$ between welded and treated series, $m$ is estimated

\begin{tabular}{|l|c|c|c|}
\hline & $\begin{array}{c}\text { post-weld } \\
\text { treatment }\end{array}$ & $\begin{array}{c}\text { as-weld } \\
\text { treatment }\end{array}$ & Post-weld/as-weld \\
\hline $\mathrm{FAT}_{95 \%}(\mathrm{t}=8 \mathrm{~mm})$ & 244 & 138 & 1.77 \\
\hline
\end{tabular}

\section{Calculation}

When the fatigue class based on free slope, there is $77 \%$ improvement can be obtained by using post-treatment. The improvement of fatigue strength based on free slope is larger than the one based on a fixed slope. 


\section{Appendix X}

Table 1 One-sided tolerance limit factors $k$ for a normal distribution for $\gamma \%=\mathbf{9 0} \%$

Degree of freedom $f=n-1 \quad$ (Schneider and Maddox, 2006)

\begin{tabular}{|c|c|c|}
\hline Sample size $n$ & Value of $k$ for $p \%=95 \%$ & Value of $k$ for $p \%=97.5 \%$ \\
\hline 2 & 13.090 & 15.586 \\
\hline 3 & 5.311 & 6.244 \\
\hline 4 & 3.975 & 4.637 \\
\hline 5 & 3.401 & 3.983 \\
\hline 6 & 3.093 & 3.621 \\
\hline 7 & 2.893 & 3.389 \\
\hline 8 & 2.754 & 3.227 \\
\hline 9 & 2.650 & 3.106 \\
\hline 10 & 2.568 & 3.011 \\
\hline 11 & 2.503 & 2.936 \\
\hline 12 & 2.448 & 2.872 \\
\hline 13 & 2.403 & 2.820 \\
\hline 14 & 2.363 & 2.774 \\
\hline 15 & 2.329 & 2.735 \\
\hline 16 & 2.299 & 2.700 \\
\hline 17 & 2.272 & 2.670 \\
\hline 18 & 2.249 & 2.643 \\
\hline 19 & 2.228 & 2.618 \\
\hline 20 & 2.208 & 2.597 \\
\hline 21 & 2.190 & 2.575 \\
\hline 22 & 2.174 & 2.557 \\
\hline 23 & 2.159 & 2.540 \\
\hline 24 & 2.145 & 2.525 \\
\hline 25 & 2.132 & 2.510 \\
\hline 30 & 2.080 & 2.450 \\
\hline 35 & 2.041 & 2.406 \\
\hline 40 & 2.010 & 2.371 \\
\hline 45 & 1.986 & 2.344 \\
\hline 50 & 1.965 & 2.320 \\
\hline 60 & 1.933 & 2.284 \\
\hline 70 & 1.909 & 2.257 \\
\hline 80 & 1.890 & 2.235 \\
\hline
\end{tabular}




\begin{tabular}{|c|c|c|}
\hline 90 & 1.874 & 2.217 \\
\hline 100 & 1.861 & 2.203 \\
\hline 120 & 1.841 & 2.179 \\
\hline 145 & 1.821 & 2.158 \\
\hline 300 & 1.765 & 2.094 \\
\hline 500 & 1.736 & 2.062 \\
\hline$\infty$ & 1.645 & 1.960 \\
\hline
\end{tabular}




\section{Appendix XI}

Table 2 One-sided tolerance limit factors $k$ for a normal distribution for $\gamma \%=\mathbf{9 0} \%$

Degree of freedom $f=n-2$

\begin{tabular}{|c|c|c|}
\hline Sample size $n$ & Value of $k$ for $p=95 \%$ & Value of $k$ for $p=97.5 \%$ \\
\hline 3 & 13.080 & 15.587 \\
\hline 4 & 5.251 & 4.193 \\
\hline 5 & - & 5.226 \\
\hline 6 & 4.204 & 4.636 \\
\hline 7 & 3.754 & 4.259 \\
\hline 8 & 3.467 & 4.000 \\
\hline 9 & 3.267 & 3.807 \\
\hline 10 & 3.117 & 3.655 \\
\hline 11 & 3.000 & 3.113 \\
\hline 18 & 2.575 & 2.668 \\
\hline 38 & 2.221 & 2.290 \\
\hline 146 & 1.917 & 1.960 \\
\hline$\infty$ & 1.650 & \\
\hline
\end{tabular}




\section{AppendixXII}

Table3 fatigue testing data from Laboratory of Fatigue and Strength in LUT

\begin{tabular}{|c|c|c|c|c|c|c|}
\hline & $t$ & $R$ & $\Delta \sigma_{S R}$ & $N$ & $\operatorname{FAT}(m=3)$ & Observation \\
\hline UIT-1 & 5 & 0.1 & 349 & 596082 & 233 & UITG \\
\hline UIT-2 & 5 & 0.1 & 318 & 310170 & 171 & UITG \\
\hline UIT-3 & 5 & 0.1 & 324 & 620074 & 219 & UITG \\
\hline UIT-4 & 5 & 0.1 & 327 & 505913 & 207 & UITG \\
\hline UIT-5 & 5 & 0.1 & 318 & 781200 & 232 & UITG \\
\hline UIT-6 & 5 & Ohta & 333 & 298108 & 177 & UITG \\
\hline UIT-7 & 5 & Ohta & 338 & 473704 & 209 & UITG \\
\hline UIT-8 & 5 & Ohta & 327 & 980692 & 258 & UITG \\
\hline UIT-9 & 5 & Ohta & 297 & 333199 & 163 & UITG \\
\hline UIT-10 & 5 & Ohta & 295 & 1163070 & 246 & UITG \\
\hline AW-11 & 5 & 0.1 & 180 & 599377 & 120 & WT \\
\hline AW-12 & 5 & 0.1 & 221 & 422755 & 131 & WT \\
\hline AW-13 & 5 & 0.1 & 127 & 2173795 & 131 & WT \\
\hline AW-14 & 5 & 0.1 & 132 & 1313035 & 115 & WT \\
\hline AW-15 & 5 & 0.1 & 204 & 480284 & 127 & WT \\
\hline AW-16 & 5 & 0.1 & 173 & 6814655 & - & RO \\
\hline AW-17 & 5 & 0.1 & 134 & 3086407 & - & RO \\
\hline UIT-18 & 8 & 0.1 & 288 & 1902884 & 283 & UITG \\
\hline UIT-19 & 8 & 0.1 & 369 & 441958 & 223 & UITG \\
\hline UIT-20 & 8 & 0.1 & 383 & 407610 & 225 & UITG \\
\hline UIT-21 & 8 & 0.1 & 335 & 588203 & 223 & UITG \\
\hline UIT-22 & 8 & 0.1 & 338 & 1892369 & 332 & UITG \\
\hline UIT-23 & 8 & Ohta & 360 & 256226 & 181 & UITG \\
\hline UIT-24 & 8 & Ohta & 392 & 393186 & 230 & UITG \\
\hline UIT-25 & 8 & Ohta & 367 & 247240 & 183 & UITG \\
\hline UIT-26 & 8 & Ohta & 379 & 205424 & 177 & UITG \\
\hline UIT-27 & 8 & Ohta & 336 & 254817 & 169 & UITG \\
\hline AW-28 & 8 & 0.1 & 193 & 485897 & 120 & WT \\
\hline AW-29 & 8 & 0.1 & 193 & 640024 & 132 & WT \\
\hline AW-30 & 8 & 0.1 & 161 & 1257193 & 138 & WT \\
\hline AW-31 & 8 & 0.1 & 180 & 1091393 & 147 & WT \\
\hline AW-32 & 8 & 0.1 & 169 & 1199013 & 143 & WT \\
\hline AW-33 & 8 & 0.1 & 153 & 3453562 & - & RO \\
\hline
\end{tabular}

